Prepared in cooperation with the Alaska Department of Transportation and Public Facilities

\title{
Measurement of Long-Term Channel Change Through Repeated Cross-Section Surveys at Bridge Crossings in Alaska
}

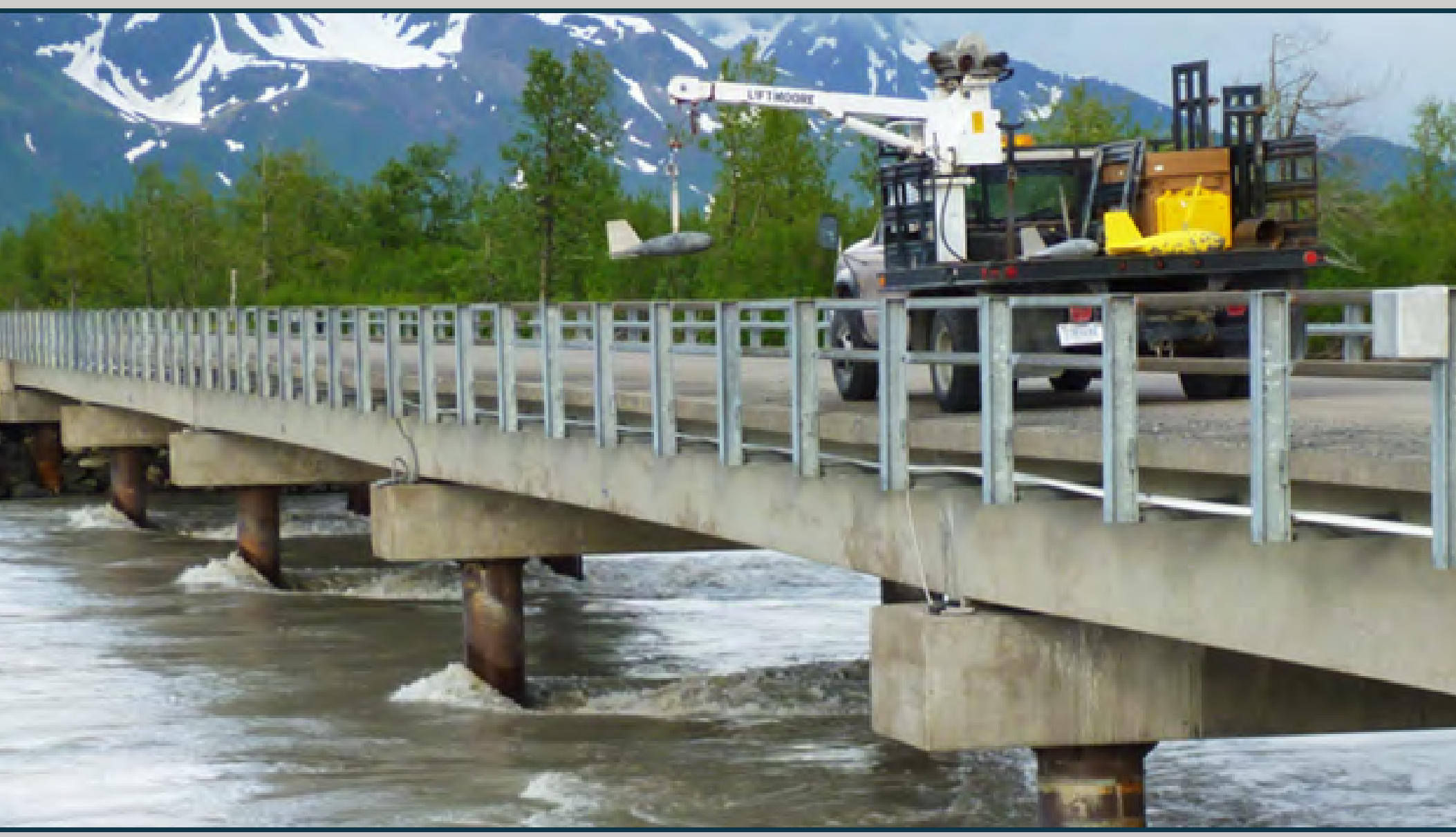

Open-File Report 2019-1028

U.S. Department of the Interior

U.S. Geological Survey 
Cover: Upstream view of a soundings measurement being taken at bridge 339 on the Copper River Delta, Alaska. Photograph by Jeffrey Conaway, U.S. Geological Survey, 2010. 


\section{Measurement of Long-Term Channel Change Through Repeated Cross-Section Surveys at Bridge Crossings in Alaska}

By Karenth L. Dworsky and Jeffrey S. Conaway

Prepared in cooperation with the Alaska Department of Transportation and Public Facilities

Open-File Report 2019-1028

U.S. Department of the Interior

U.S. Geological Survey 


\title{
U.S. Department of the Interior DAVID BERNHARDT, Acting Secretary
}

\author{
U.S. Geological Survey \\ James F. Reilly II, Director
}

U.S. Geological Survey, Reston, Virginia: 2019

For more information on the USGS-the Federal source for science about the Earth, its natural and living resources, natural hazards, and the environment-visit https://www.usgs.gov/ or call 1-888-ASK-USGS (1-888-275-8747).

For an overview of USGS information products, including maps, imagery, and publications, visit https:/store.usgs.gov.

Any use of trade, firm, or product names is for descriptive purposes only and does not imply endorsement by the U.S. Government.

Although this information product, for the most part, is in the public domain, it also may contain copyrighted materials as noted in the text. Permission to reproduce copyrighted items must be secured from the copyright owner.

Suggested citation:

Dworsky, K.L., and Conaway, J.S., 2019, Measurement of long-term channel change through repeated crosssection surveys at bridge crossings in Alaska: U.S. Geological Survey Open-File Report 2019-1028, 118 p., https://doi.org/10.3133/ofr20191028.

ISSN 2331-1258 (online) 


\section{Contents}

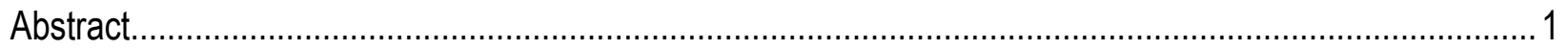

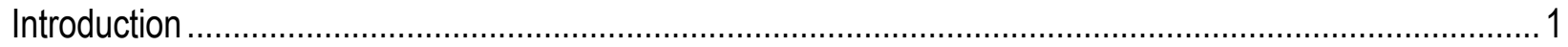

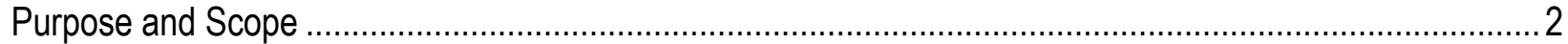

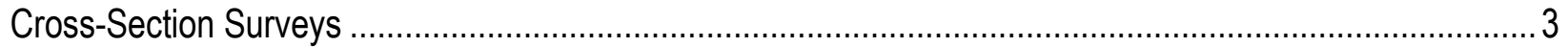

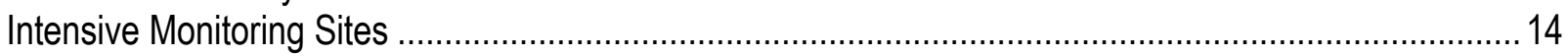

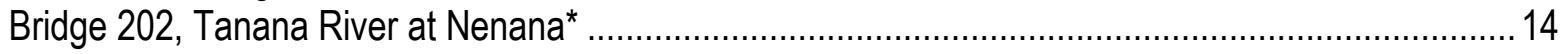

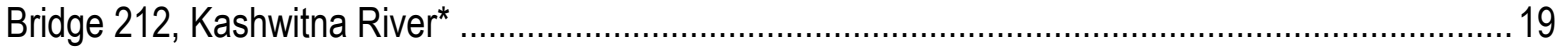

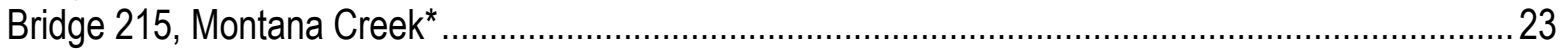

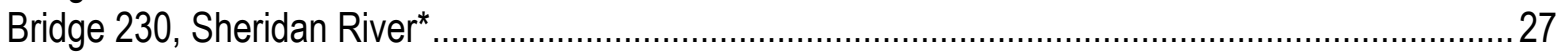

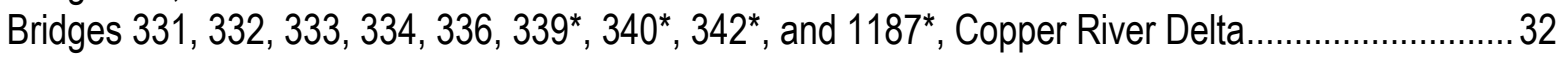

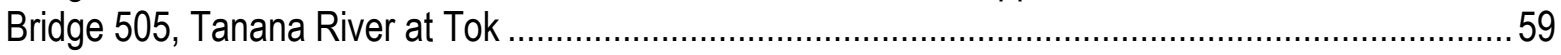

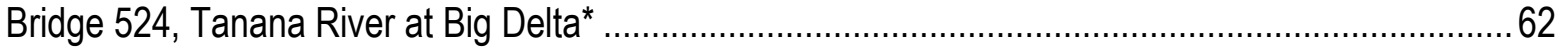

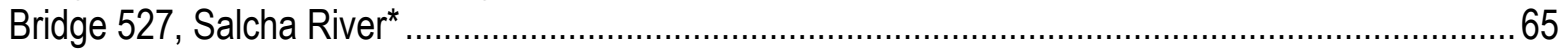

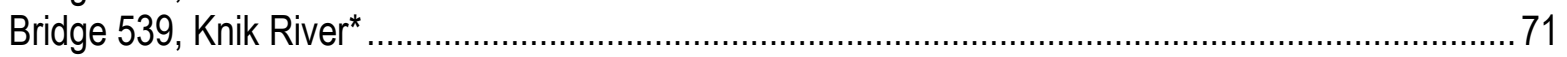

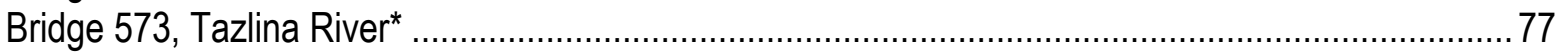

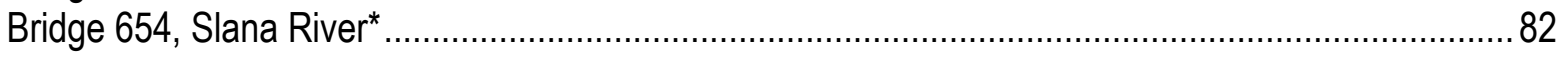

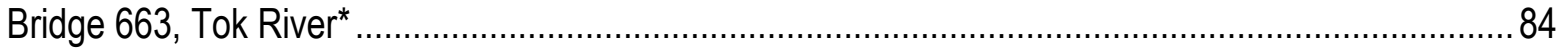

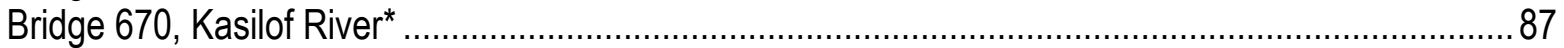

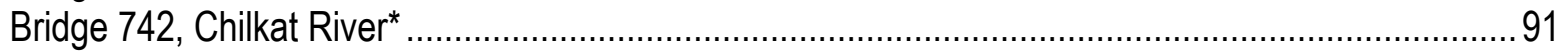

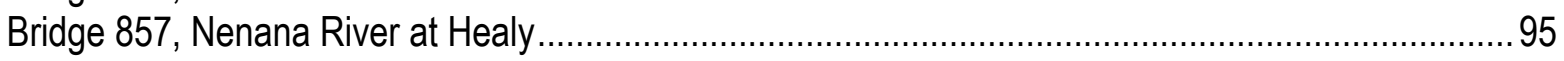

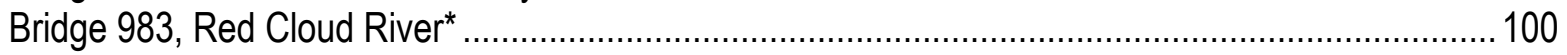

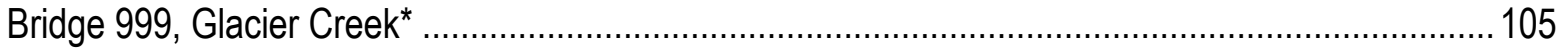

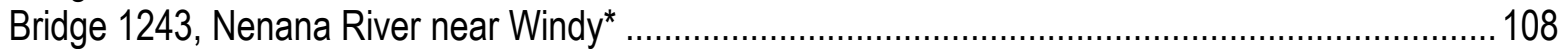

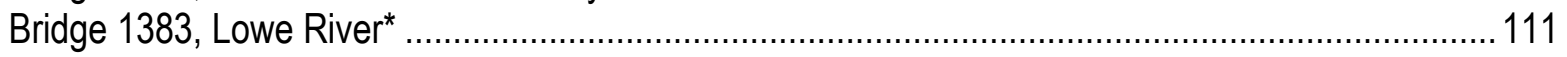

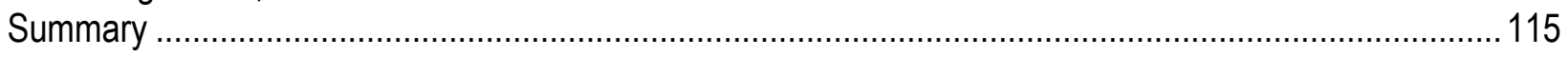

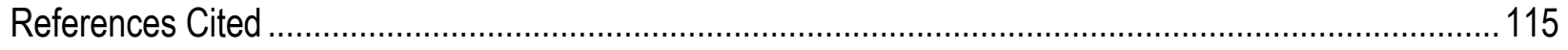

Appendix 1. Tabular Data for All Cross Sections Collected for this Study ..........................................117

Appendix 2. Cross Sections of Bed Elevation and Bridge Geometry for Bridges Not Discussed in this Report 


\section{Figures}

Figure 1. Surveyed cross sections relative to as-built bud elevations, defined as either fill or scour............... 4

Figure 2. Map showing locations of bridges in the road system in Alaska selected for long term monitoring to assess scour susceptibility

Figure 3. Graph showing sounding-based stream stability at 76 river- and stream-spanning bridges in Alaska

Figure 4. Cross sections showing upstream soundings at bridge 202, Tanana River at Nenana, Alaska, 1998-2004.

Figure 5. Cross sections showing upstream soundings at bridge 202, Tanana River at Nenana, Alaska, 2005-09

Figure 6. Cross sections showing upstream soundings at bridge number 202, Tanana River at Nenana,

Alaska, 2010-14

Figure 7. Cross section showing upstream soundings at bridge 202, Tanana River at Nenana, Alaska,

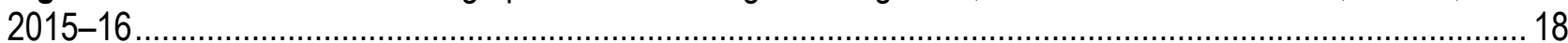

Figure 8. Cross sections showing upstream soundings at bridge 212, Kashwitna River, Alaska, 2005-10 ... 20 Figure 9. Cross sections showing upstream soundings at bridge 212, Kashwitna River, Alaska, 2011-13 ... 21 Figure 10. Cross sections showing upstream soundings at bridge 212, Kashwitna River, Alaska, 2014-16

Figure 11. Cross sections showing upstream soundings at bridge 215, Montana Creek, Alaska, 2004-11

Figure 12. Cross sections showing upstream soundings at bridge 215, Montana Creek, Alaska, 2012-14

Figure 13. Cross sections showing upstream soundings at bridge 215, Montana Creek, Alaska, 2015-16.

Figure 14. Cross sections showing upstream soundings at bridge 230, Sheridan River, Alaska, 2005-09 ...28

Figure 15. Cross sections showing upstream soundings at bridge 230, Sheridan River, Alaska, 2010-13 ... 29

Figure 16. Cross sections showing upstream soundings at bridge 230, Sheridan River, Alaska, 2014-16 ... 30

Figure 17. Cross sections showing downstream soundings at bridge 230 , Sheridan River, Alaska, 2004-15

Figure 18. Cross sections showing downstream soundings at bridge 331, Copper River Delta, Alaska, 2005-16.

Figure 19. Cross sections showing upstream soundings at bridge 331, Copper River Delta, Alaska, 2005-08

Figure 20. Cross sections showing downstream soundings at bridge 332, Copper River Delta, Alaska, 2006-13

Figure 21. Cross sections showing upstream soundings at bridge 332, Copper River Delta, Alaska, 2008

Figure 22. Cross sections showing upstream soundings at bridge 333, Copper River Delta, Alaska, 2008-11

Figure 23. Cross sections showing downstream soundings at bridge 333, Copper River Delta, Alaska, 2006-07.

Figure 24. Cross sections showing downstream soundings at bridge 334, Copper River Delta, Alaska, 2006-10.

Figure 25. Cross sections showing upstream soundings at bridge 334, Copper River Delta, Alaska, 2008-11 
Figure 26. Cross sections showing downstream soundings at bridge 336, Copper River Delta, Alaska, 2007-09.

Figure 27. Cross sections showing upstream soundings at bridge 339, Copper River Delta, Alaska, 2004-05.

Figure 28. Cross sections showing upstream soundings at bridge 339, Copper River Delta, Alaska, 2006-07.

Figure 29. Cross sections showing upstream soundings at bridge 339, Copper River Delta, Alaska, 2008-09

Figure 30. Cross sections showing upstream soundings at bridge 339, Copper River Delta, Alaska, 2010

Figure 31. Cross sections showing upstream soundings at bridge 339, Copper River Delta, Alaska, 2011-13

Figure 32. Cross sections showing downstream soundings at bridge 339, Copper River Delta, Alaska, 2010.

Figure 33. Cross sections showing downstream soundings at bridge 339, Copper River Delta, Alaska, 2011

Figure 34. Cross sections showing downstream soundings at bridge 340, Copper River Delta, Alaska, 2004-10.

Figure 35. Cross sections showing upstream soundings at bridge 340, Copper River Delta, Alaska, 2005-11

Figure 36. Cross sections showing downstream soundings at bridge 342, Copper River Delta, Alaska, 2005-07

Figure 37. Cross sections showing downstream soundings at bridge 342, Copper River Delta, Alaska, 2008-09.

Figure 38. Cross sections showing downstream soundings at bridge 342, Copper River Delta, Alaska, 2010-11

Figure 39. Cross sections showing upstream soundings at bridge 342, Copper River Delta, Alaska, 2005-14.

Figure 40. Cross sections showing downstream soundings at bridge 1187, Copper River Delta, Alaska, 2005-07.

Figure 41. Cross sections showing downstream soundings at bridge 1187, Copper River Delta, Alaska, 2009-12

Figure 42. Cross sections showing downstream soundings at bridge 1187, Copper River Delta, Alaska, 2013-16.

Figure 43. Cross sections showing upstream soundings at bridge 1187, Copper River Delta, Alaska, 2005-08

Figure 44. Cross sections showing upstream soundings at bridge 505, Tanana River at Tok, Alaska, 2002-07.

Figure 45. Cross sections showing upstream soundings at bridge 505, Tanana River at Tok, Alaska, 2008-10

Figure 46. Cross sections showing upstream soundings at bridge 524, Tanana River at Big Delta, Alaska, 1971-2014

Figure 47. Cross sections showing downstream soundings at bridge 524, Tanana River at Big Delta, Alaska, 2005-10

Figure 48. Cross sections showing upstream soundings at bridge 527, Salcha River, Alaska, 1995-2003.

Figure 49. Cross sections showing upstream soundings at bridge 527, Salcha River, Alaska, 2005-11 ...... 67 
Figure 50. Cross sections showing upstream soundings at bridge 527, Salcha River, Alaska, 2012-14.......68

Figure 51. Cross sections showing upstream soundings at bridge 527, Salcha River, Alaska, 2015-16.......69 Figure 52. Cross sections showing downstream soundings at bridge 527, Salcha River, Alaska, 2003-16. 70

Figure 53. Cross sections showing upstream soundings at bridge 539, Knik River, Alaska, 2005-06 ............72 Figure 54. Cross sections showing upstream soundings at bridge 539 Knik River, Alaska, 2007-08............73 Figure 55. Cross sections showing upstream soundings at bridge 539, Knik River, Alaska, 2009-10...........74 Figure 56. Cross sections showing upstream soundings at bridge 539, Knik River, Alaska, 2011-13.......... 75 Figure 57. Cross sections showing upstream soundings at bridge 539, Knik River, Alaska, 2015-16 .......... 76 Figure 58. Cross sections showing upstream soundings at bridge 573, Tazlina River, Alaska,

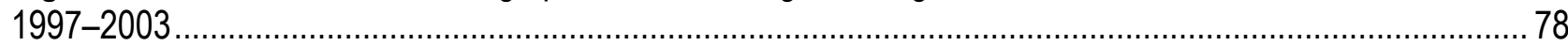

Figure 59. Cross sections showing upstream soundings at bridge 573, Tazlina River, Alaska, 2004-10 ......79 Figure 60. Cross sections showing upstream soundings at bridge 573, Tazlina River, Alaska, 2012-16 ...... 80 Figure 61. Cross sections showing downstream soundings at bridge 573, Tazlina River, Alaska,

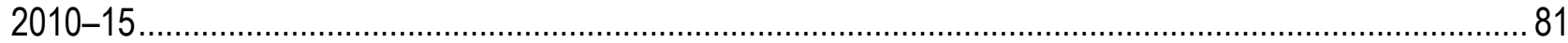

Figure 62. Cross sections showing upstream soundings at bridge 654, Slana River, Alaska, 2002-11 ........83

Figure 63. Cross sections showing upstream soundings at bridge 663, Tok River, Alaska, 2002-09........... 85

Figure 64. Cross sections showing upstream soundings at bridge 663, Tok River, Alaska, 2010-14........... 86

Figure 65. Cross sections showing upstream soundings at bridge 670, Kasilof River, Alaska, 2002-05 ....... 88

Figure 66. Cross sections showing upstream soundings at bridge 670, Kasilof River, Alaska, 2007-11 .......89

Figure 67. Cross sections showing upstream soundings at bridge 670, Kasilof River, Alaska, 2012-16 .......90

Figure 68. Cross sections showing upstream soundings at bridge 742, Chilkat River, Alaska, 2001-11 ...... 92

Figure 69. Cross sections showing upstream soundings at bridge 742, Chilkat River, Alaska, 2013-16 .......93

Figure 70. Cross sections showing downstream soundings at bridge 742, Chilkat River, Alaska, 2001-05.

Figure 71. Cross sections showing downstream soundings at bridge 857, Nenana River at Healy, Alaska, 2003-04. 96

Figure 72. Cross sections showing downstream soundings at bridge 857, Nenana River at Healy, Alaska, 2005-06.

Figure 73. Cross sections showing downstream soundings at bridge 857, Nenana River at Healy, Alaska, 2007-11 98

Figure 74. Cross sections showing upstream soundings at bridge 857, Nenana River at Healy, Alaska, 1999-2015...

Figure 75. Cross sections showing upstream soundings at bridge 983, Red Cloud River, Alaska, 2005-10

Figure 76. Cross sections showing upstream soundings at bridge 983, Red Cloud River, Alaska, $2011-14$

Figure 77. Cross sections showing upstream soundings at bridge 983, Red Cloud River, Alaska, 2015-16.

Figure 78. Cross sections showing downstream soundings at bridge 983, Red Cloud River, Alaska, 2009-16

Figure 79. Cross sections showing upstream soundings at bridge 999, Glacier Creek, Alaska, 1999-2010. 106

Figure 80. Cross sections showing upstream soundings at bridge 999, Glacier Creek, Alaska, 2011-16 ... 107 Figure 81. Cross sections showing upstream soundings at bridge 1243, Nenana River near Windy, Alaska, 2007-12. 
Figure 82. Cross sections showing upstream soundings at bridge 1243, Nenana River near Windy, Alaska, 2013-16

Figure 83. Cross sections showing upstream soundings at bridge 1383, Lowe River, Alaska, 2005-10 .... 112

Figure 84. Cross sections showing upstream soundings at bridge 1383, Lowe River, Alaska, 2011-13 .....113

Figure 85. Cross sections showing upstream soundings at bridge 1383, Lowe River, Alaska, 2014-16 ..... 114

\section{Table}

Table 1. Location of bridges on road system starting from either the southern or western direction, years of measured cross sections, and river stability.

\section{Conversion Factors}

U.S. customary units to International System of Units

\begin{tabular}{lll}
\hline \multicolumn{1}{c}{ Multiply } & By & To obtain \\
\hline & Length & \\
\hline foot $(\mathrm{ft})$ & 0.3048 & meter $(\mathrm{m})$ \\
mile $(\mathrm{mi})$ & 1.609 & kilometer $(\mathrm{km})$ \\
\hline & Flow rate & \\
\hline foot per second $(\mathrm{ft} / \mathrm{s})$ & 0.3048 & meter per second \\
& & $(\mathrm{m} / \mathrm{s})$ \\
\hline
\end{tabular}

\section{Datums}

Vertical coordinate information is site specific and, in most cases, is referenced either to as-built elevations on bridge plans (if available) or to a reference mark with an assumed elevation of 100 feet established during the survey on or near the bridge deck.

Horizontal coordinate information is referenced to the World Geodetic System of 1984 (WGS 84).

\section{Abbreviations}

ADCP acoustic Doppler current profilers

ADOT\&PF Alaska Department of Transportation and Public Facilities

USGS U.S. Geological Survey 
This page intentionally left blank 


\title{
Measurement of Long-Term Channel Change Through Repeated Cross-Section Surveys at Bridge Crossings in Alaska
}

\author{
By Karenth L. Dworsky and Jeffrey S. Conaway
}

\begin{abstract}
The U.S. Geological Survey (USGS) has been working with Alaska Department of Transportation and Public Facilities (ADOT\&PF) since 1993 to provide hydraulic assessments of scour for bridges throughout Alaska. The purpose of the program is to evaluate, monitor, and study streambed scour at bridges in Alaska; this includes surveying streambed elevations at regular intervals and monitoring real-time bed elevation changes. Over the duration of the scour program (1994-2017), repeated cross sections have been surveyed along the lengths of 76 bridges. Channel soundings are depth-from-bridge measurements on either the upstream or downstream side of a bridge. Flow, depth, and velocity dictated whether streambed elevations were measured using either USGS sounding weights on cable reels, weighted measuring tapes, or acoustic Doppler current profilers. The soundings were done on an annual basis at most sites. In addition to annual soundings, channel soundings were made during floods or periods of scour. Results show that general scour can be uniform or non-uniform across the channel. The magnitude and distribution of scour across the channel are influenced by several factors that include streambed sediment type, degree of channel contraction at the bridge crossing, influence of instream structures, and bridge pier location and alignment. The data collected from the repeat soundings can be used to identify long-term aggradation or degradation of the streambed, as well as seasonal changes in streambed elevations.
\end{abstract}

\section{Introduction}

The U.S. Geological Survey (USGS) first began investigating scour at bridges in Alaska in 1965 (Norman, 1975). Since 1994, USGS and the Alaska Department of Transportation and Public Facilities (ADOT\&PF) have been collaborating to assess, model, and monitor streambed scour at bridges in Alaska. This collaboration has generated long-term data sets of hydraulic and geomorphic variable, hydraulic models, and publications of streambed scour assessments (Heinrichs and others, 2001; Conaway, 2004; Conaway and Schauer, 2012; Beebee and Schauer, 2015; Beebee and others, 2017). Bridges with abutment or pier foundations determined to be unstable owing to (1) observed scour at the site or (2) the potential for severe streambed scour as determined from a scour evaluation are designated as scour critical (Arneson and others, 2012). Federal recommendations for a plan of action for scourcritical bridges include the development and implementation of a scour monitoring and inspection program until the bridge is replaced or until scour countermeasures are designed and installed. 
The USGS initially evaluated the susceptibility of Alaska bridges to scour in a two-phase analysis following Federal guidelines outlined in the Hydrologic Engineering Circular No. 18 (HEC-18) (Arneson and others, 2012) and earlier versions of that circular. The first phase determined scour based on channel geometry from bridge plans and used either assumed or (when available) measured hydraulic properties (Heinrichs and other, 2001). The second phase determined scour based on surveys of channel geometry and measured hydraulic properties (Conaway, 2004). These analyses and others completed by ADOT\&PF identified more than 20 bridges as scour critical or recommended for further analysis and data collection. Since the completion of the second phase scour evaluations, many bridges have been removed from the scour-critical list for one or more of the following factors: (1) replacement of the bridge, (2) lack of observed scour, (3) installation of scour countermeasures, (4) and road closure. The USGS currently (2019) operates a real-time monitoring network of pier-mounted sonars that measure streambed elevations at 17 scour-critical bridges (http://ak.water.usgs.gov/usgs_scour).

In addition to the real-time monitoring, the USGS collects cross-sectional information at bridges to monitor streambed elevation changes of the entire cross section and to track long-term bed elevation changes at sites without real-time monitoring instrumentation. Channel soundings are depth-from-bridge measurements on either the upstream or downstream side of a bridge. For scour-critical sites, soundings typically are obtained annually. However, for sites that are not considered scour critical, soundings can be measured at intervals that are several years apart. The data from the repeat soundings are used to identify long-term aggradation or degradation of the streambed, as well as seasonal changes in streambed elevations.

\section{Purpose and Scope}

This report presents repeated cross-section surveys measured by the USGS at select bridges in Alaska during 1971-2016. These bridges were identified by previous USGS scour evaluations or by ADOT\&PF as being susceptible to streambed scour. Cross-section surveys are used to identify channel stability, long-term aggradation or degradation of the streambed, and seasonal changes in streambed elevation; and to fulfill the Federal recommendation for scour monitoring at scour-critical sites. More detail is included for sites that have been part of the real-time streambed scour monitoring network. The scope of this report is limited to a discussion of methods and presentation of data; interpretation of these data is presented in other USGS publications (Heinrichs and others, 2001; Conaway, 2004; Conaway and Schauer, 2012; Beebee and Schauer, 2015; Beebee and others, 2017). 


\section{Cross-Section Surveys}

Streambed scour, and fill are quantified relative to a base or starting elevation. The reference surface for general scour, contraction, or scour from flow around a bend is the cross section that was surveyed when the bridge was built. This section is referred to as the as-built cross section. Surveyed bed elevations above this as-built cross section are considered fill and those below it are considered scour (fig. 1). General scour can be uniform or non-uniform across the channel. The magnitude and distribution of scour across the channel are influenced by several factors that include streambed sediment type, degree of channel contraction at the bridge crossing, influence of in-stream structures, and bridge pier location and alignment.

Streambed depths were recorded along the lengths of 76 bridges (fig. 2). Selection of bridges was determined by ADOT\&PF and the USGS from results of scour susceptibility evaluations done prior to this study (Norman, 1975; Heinrichs and others, 2001; Conaway, 2004). Flow depth and velocity dictated whether streambed elevations were measured using either USGS sounding weights on cable reels, weighted measuring tapes, or acoustic Doppler current profilers (ADCP). Depth measurements of every method may be affected by wind and water velocity and sediment movement at the time of the survey. However, repeat soundings at stable sites agree within $0.1-0.3 \mathrm{ft}$. 


\section{As-built and bridge}

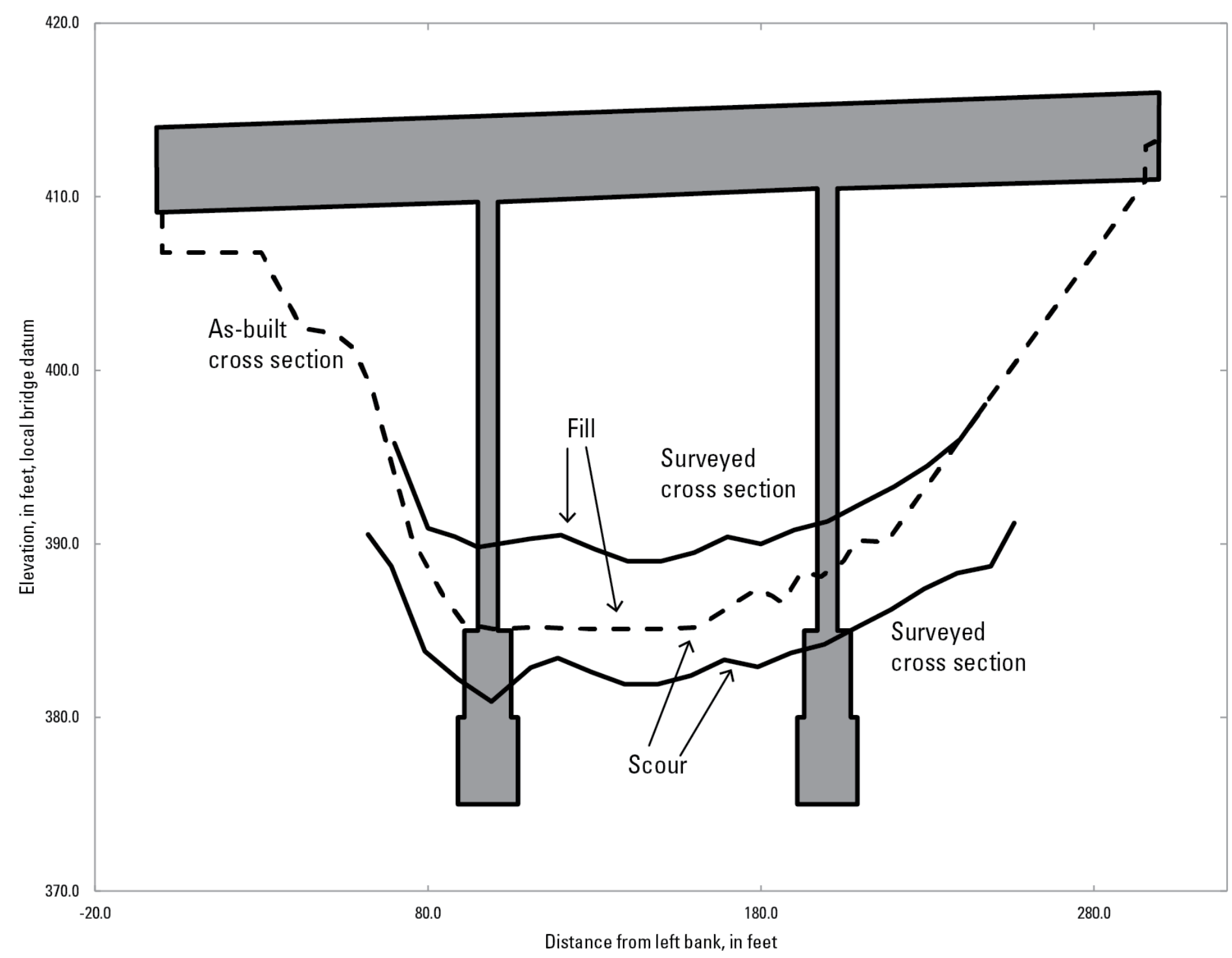

Figure 1. Surveyed cross sections relative to as-built bud elevations, defined as either fill or scour. 


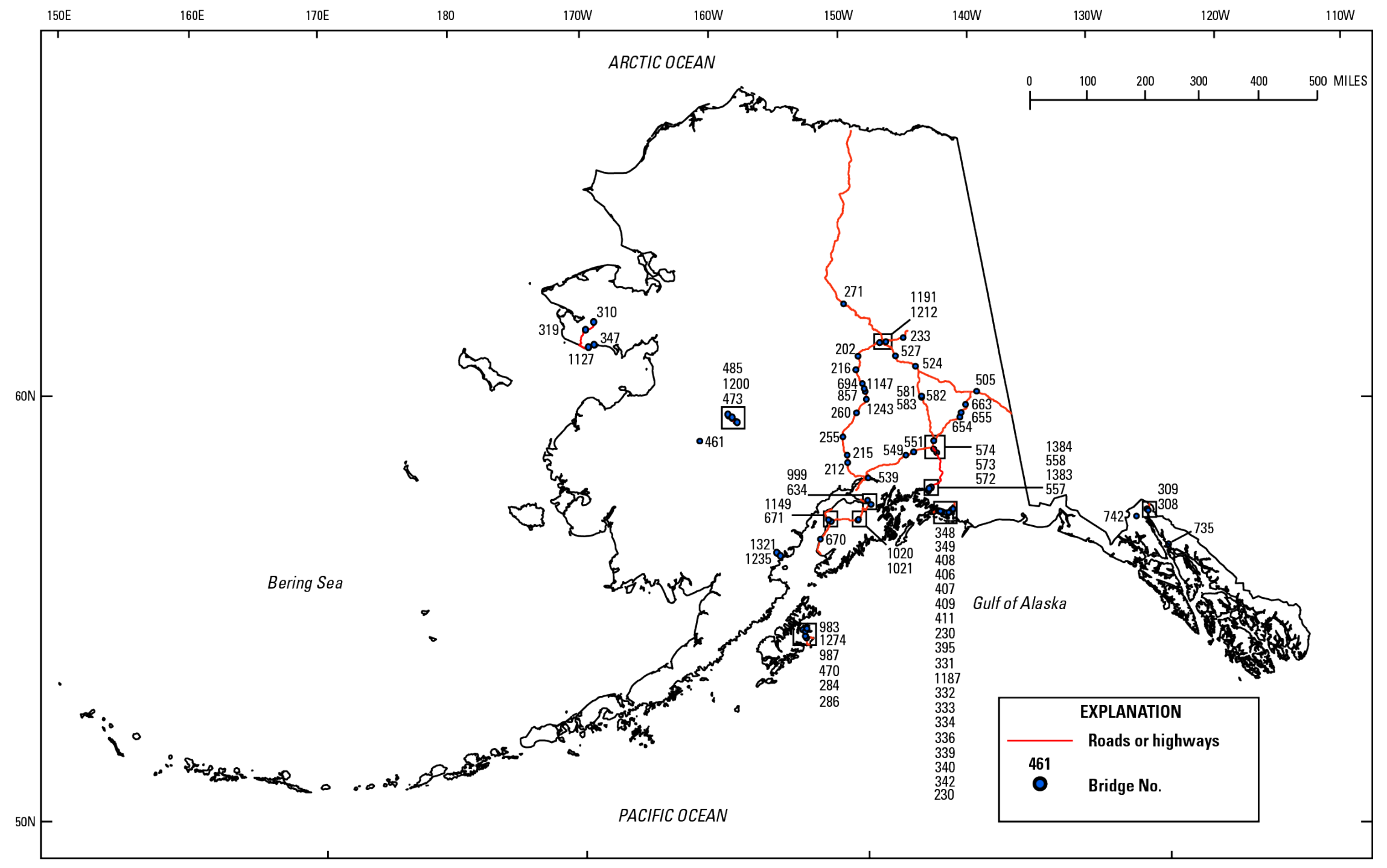

Figure 2. Map showing locations of bridges in the road system in Alaska selected for long term monitoring to assess scour susceptibility. 
Whenever possible, sounding depths were measured on the upstream side of the bridges to record the areas of greatest scour, which typically is near the noses of the bridge piers. Streambed depths were referenced to the bridge-deck elevations given on the bridge construction plans provided by ADOT\&PF. Streambed depth changes over the length of the study can then be compared to the original streambed surveys done when the bridges were constructed. In a few cases where the original bridge plans are missing, the streambed depths are referenced to an arbitrary bridge deck elevation of $100 \mathrm{ft}$. Horizontal distances are referenced from the start of the bridge on the left bank. The start of a bridge is identified as the expansion joint between the bridge deck and the adjoining road.

The soundings were done on an annual basis at most sites. In addition to annual soundings, channel soundings were made during floods or periods of scour. Dates and locations of crosssectional surveys are included in table 1 . Several of the bridges were surveyed multiple times in a year at different river stages to distinguish between short-term or seasonal scour and fill compared to long-term aggradation and degradation. Minimum and maximum low bed elevations were determined for each survey for comparison purposes (table 1). 
Table 1. Location of bridges on road system starting from either the southern or western direction, years of measured cross sections, and river stability.

\begin{tabular}{|c|c|c|c|c|c|c|c|c|}
\hline \multirow{2}{*}{$\begin{array}{l}\text { Bridge No. } \\
\text { (fig. 2) }\end{array}$} & \multirow[b]{2}{*}{ Bridge name } & \multirow[b]{2}{*}{ Highway/road } & \multirow{2}{*}{$\begin{array}{l}\text { Location } \\
\text { (mile) }\end{array}$} & \multirow{2}{*}{$\begin{array}{l}\text { Soundings } \\
\text { (years) }\end{array}$} & \multicolumn{3}{|c|}{ Low bed elevations (feet) } & \multirow{2}{*}{$\begin{array}{c}\text { Stable/ } \\
\text { unstable }\end{array}$} \\
\hline & & & & & Minimum & Maximum & $\begin{array}{c}\text { Change/ } \\
\text { 10-foot width }\end{array}$ & \\
\hline 202 & Tanana River at Nenana & Parks Highway & 270.7 & $1998,2003-16$ & 333.1 & 324.6 & 0.13 & Stable \\
\hline 212 & Kashwitna River & Parks Highway & 48.3 & $2005-16$ & 158.1 & 160.8 & 0.16 & Stable \\
\hline 215 & Montana Creek & Parks Highway & 61.6 & $2004-16$ & 243.3 & 239.8 & 0.44 & Unstable \\
\hline 216 & Nenana River at Rex & Parks Highway & 240.3 & 2004-09, 2011 & 646.4 & 696.3 & 0.28 & Stable \\
\hline 230 & Sheridan River & $\begin{array}{l}\text { Copper River } \\
\text { Highway }\end{array}$ & 39.5 & $2004-16$ & 14.8 & 34.4 & 1.12 & Unstable \\
\hline 233 & Chena at Two Rivers & $\begin{array}{c}\text { Chena River } \\
\text { Highway }\end{array}$ & 14.8 & $2001-06$ & 723.4 & 727.9 & 0.43 & Unstable \\
\hline 255 & Chulitna River & Parks Highway & 98.3 & $2002-11$ & 475.6 & 481.6 & 0.10 & Stable \\
\hline 260 & $\begin{array}{l}\text { East Fork of the Chulitna } \\
\text { River }\end{array}$ & Parks Highway & 150.8 & $2010-11$ & 1751.4 & $1,751.4$ & 0.00 & Stable \\
\hline 271 & Yukon River & Dalton Highway & 55.8 & 1993,2014 & 235.0 & 237.0 & 0.01 & Stable \\
\hline 284 & American River & Chiniak Highway & 10.5 & $2005-09,2011$ & ${ }^{1} 84.0$ & 85.2 & 0.09 & Stable \\
\hline 286 & Deadman Creek & Chiniak Highway & 18.7 & 2005-09, 2011 & ${ }^{1} 87.2$ & 88.0 & 0.18 & Stable \\
\hline 308 & Skagway River & Klondike Highway & 1.8 & $2001,2004-08,2011$ & 52.0 & 54.8 & 0.07 & Stable \\
\hline 309 & Taiya River & Skagway/Dyea Road & 7.1 & 2011,2015 & ${ }^{1} 78.3$ & 78.5 & 0.01 & Stable \\
\hline 310 & Pilgrim River & Nome-Taylor Road & 60.5 & 2007,2011 & 79.8 & 80.5 & 0.05 & Stable \\
\hline 319 & Grand Central River & Nome-Taylor Road & 35.5 & 2007,2011 & ${ }^{1} 75.5$ & 76.3 & 0.10 & Stable \\
\hline
\end{tabular}




\begin{tabular}{|c|c|c|c|c|c|c|c|c|}
\hline \multirow{2}{*}{$\begin{array}{l}\text { Bridge No. } \\
\text { (fig. 2) }\end{array}$} & \multirow[b]{2}{*}{ Bridge name } & \multirow[b]{2}{*}{ Highway/road } & \multirow{2}{*}{$\begin{array}{l}\text { Location } \\
\text { (mile) }\end{array}$} & \multirow{2}{*}{$\begin{array}{l}\text { Soundings } \\
\text { (years) }\end{array}$} & \multicolumn{3}{|c|}{ Low bed elevations (feet) } & \multirow{2}{*}{$\begin{array}{c}\text { Stable/ } \\
\text { unstable }\end{array}$} \\
\hline & & & & & Minimum & Maximum & $\begin{array}{c}\text { Change/ } \\
\text { 10-foot width }\end{array}$ & \\
\hline 331 & Copper River Delta & $\begin{array}{l}\text { Copper River } \\
\text { Highway }\end{array}$ & 26.7 & $\begin{array}{c}2005,2007-09,2011- \\
13,2015,2016\end{array}$ & -2.1 & -0.2 & 0.03 & Stable \\
\hline 332 & Copper River Delta & $\begin{array}{l}\text { Copper River } \\
\text { Highway }\end{array}$ & 27.6 & $2006-13$ & 6.8 & 10.5 & 0.06 & Stable \\
\hline 333 & Copper River Delta & $\begin{array}{l}\text { Copper River } \\
\text { Highway }\end{array}$ & 33.7 & 2006-09, 2011 & 11.5 & 15.9 & 0.34 & Stable \\
\hline 334 & Copper River Delta & $\begin{array}{l}\text { Copper River } \\
\text { Highway }\end{array}$ & 34.6 & $2006-11$ & 15.1 & 19.1 & 0.05 & Stable \\
\hline 336 & Copper River Delta & $\begin{array}{l}\text { Copper River } \\
\text { Highway }\end{array}$ & 35.6 & $2007-09$ & 32.4 & 35.5 & 0.19 & Stable \\
\hline 339 & Copper River Delta & $\begin{array}{l}\text { Copper River } \\
\text { Highway }\end{array}$ & 36.2 & $2004-13$ & -12.2 & 33.7 & 1.31 & Unstable \\
\hline 340 & Copper River Delta & $\begin{array}{l}\text { Copper River } \\
\text { Highway }\end{array}$ & 36.5 & $2004-11$ & 25.2 & 30.4 & 0.27 & Stable \\
\hline 342 & Copper River Delta & $\begin{array}{l}\text { Copper River } \\
\text { Highway }\end{array}$ & 37.0 & 2005-11, 2014 & 33.8 & -0.2 & 0.25 & Stable \\
\hline 347 & Bonanza Crossing & Nome-Teller Road & 31.6 & $2008,2010,2011$ & 26.4 & 28.7 & 0.18 & Stable \\
\hline 348 & Scott Glacier Creek & $\begin{array}{l}\text { Copper River } \\
\text { Highway }\end{array}$ & 7.5 & 2010,2011 & 23.4 & 24.4 & 0.07 & Stable \\
\hline 349 & Scott Glacier Creek & $\begin{array}{l}\text { Copper River } \\
\text { Highway }\end{array}$ & 7.6 & $\begin{array}{l}2007,2008,2010, \\
\quad 2011\end{array}$ & 24.9 & 26.5 & 0.09 & Stable \\
\hline 395 & Alaganik Slough & $\begin{array}{l}\text { Copper River } \\
\text { Highway }\end{array}$ & 22.3 & 2011,2015 & -0.7 & -0.2 & 0.05 & Stable \\
\hline 406 & Scott Glacier Creek & $\begin{array}{l}\text { Copper River } \\
\text { Highway }\end{array}$ & 9.5 & $\begin{array}{l}1996,2005-08,2010 \\
\quad 2011\end{array}$ & 35.3 & 38.6 & 0.09 & Stable \\
\hline 407 & Scott Glacier Creek & $\begin{array}{l}\text { Copper River } \\
\text { Highway }\end{array}$ & 9.7 & $\begin{array}{l}1996,2005-08,2010 \\
\quad 2011\end{array}$ & 36.9 & 39.9 & 0.19 & Stable \\
\hline 408 & Scott Glacier Creek & $\begin{array}{l}\text { Copper River } \\
\text { Highway }\end{array}$ & 10.0 & $2006-10$ & 33.8 & 38.8 & 0.43 & Unstable \\
\hline 409 & Scott Glacier Creek & $\begin{array}{l}\text { Copper River } \\
\text { Highway }\end{array}$ & 10.4 & 1996,2010 & 34.8 & 35.5 & 0.10 & Stable \\
\hline 411 & Scott Glacier Creek & $\begin{array}{l}\text { Copper River } \\
\text { Highway }\end{array}$ & 11.0 & 1996,2010 & 29.6 & 30.5 & 0.03 & Stable \\
\hline
\end{tabular}




\begin{tabular}{|c|c|c|c|c|c|c|c|c|}
\hline \multirow{2}{*}{$\begin{array}{l}\text { Bridge No. } \\
\text { (fig. 2) }\end{array}$} & \multirow[b]{2}{*}{ Bridge name } & \multirow[b]{2}{*}{ Highway/road } & \multirow{2}{*}{$\begin{array}{l}\text { Location } \\
\text { (mile) }\end{array}$} & \multirow{2}{*}{$\begin{array}{l}\text { Soundings } \\
\text { (years) }\end{array}$} & \multicolumn{3}{|c|}{ Low bed elevations (feet) } & \multirow{2}{*}{$\begin{array}{c}\text { Stable/ } \\
\text { unstable }\end{array}$} \\
\hline & & & & & Minimum & Maximum & $\begin{array}{c}\text { Change/ } \\
\text { 10-foot width }\end{array}$ & \\
\hline$\overline{461}$ & Otter Creek & Iditarod Highway & 7.2 & 2007,2011 & $1-11.8$ & -11.4 & 0.11 & Stable \\
\hline 470 & Small Creek & Chiniak Highway & 9.2 & 2005-09, 2011 & 91.9 & 92.4 & 0.06 & Stable \\
\hline 473 & Gold Creek & Airfield road & 1.0 & 2007,2011 & ${ }^{1} 89.1$ & 89.8 & 0.70 & Unstable \\
\hline 485 & Spruce Creek & Sterling/Ophir road & 42.2 & $2007,2011,2015$ & 193.2 & 93.7 & 0.45 & Unstable \\
\hline 505 & Tanana River at Tok & Alaska Highway & 86.5 & $2002-10$ & $1,507.4$ & $1,526.8$ & 0.23 & Stable \\
\hline 524 & Tanana River at Big Delta & Richardson Highway & 208.3 & $\begin{array}{l}1971,2004-10, \\
2012-14\end{array}$ & 957.7 & 968.1 & 0.18 & Stable \\
\hline 527 & Salcha River & Richardson Highway & 324.7 & $1995,2001-16$ & 626.3 & 634.5 & 0.28 & Stable \\
\hline 539 & Knik River & Old Glen Highway & 8.9 & $2005-13,2015-16$ & 17.5 & 31.2 & 0.33 & Stable \\
\hline 557 & Lowe River & Richardson Highway & 18.8 & $\begin{array}{l}1998,2006-10,2012 \\
2014-16\end{array}$ & 365.8 & 373.3 & 0.18 & Stable \\
\hline 558 & Lowe River & Richardson Highway & 20.3 & $2006-09$ & 429.4 & 432.8 & 0.09 & Stable \\
\hline 572 & Kluitna River & $\begin{array}{l}\text { Old Richardson } \\
\text { Highway }\end{array}$ & 0.5 & 2010,2012 & $1,009.5$ & $1,010.2$ & 0.04 & Stable \\
\hline 573 & Tazlina River & Richardson Highway & 113.4 & $\begin{array}{l}1997,1999,2002-07 \\
2012-13,2015 \\
2016\end{array}$ & $1,093.3$ & $1,112.4$ & 0.50 & Unstable \\
\hline 574 & Gulkana River & Richardson Highway & 129.8 & $2001,2002,2004-07$ & $1,362.7$ & $1,365.6$ & 0.10 & Stable \\
\hline 581 & Upper Miller Creek & Richardson Highway & 217.9 & $2004-10$ & $2,484.7$ & $2,485.7$ & 0.07 & Stable \\
\hline 582 & Lower Miller Creek & Richardson Highway & 219.5 & 2004-07, 2009, 2010 & $2,459.9$ & $2,462.4$ & 0.25 & Stable \\
\hline 583 & Castner Creek & Richardson Highway & 219.9 & $2004-10$ & $2,466.7$ & $2,468.4$ & 0.17 & Stable \\
\hline 634 & Twenty Mile River & Seward Highway & 79.9 & $2010-13,2015,2016$ & 1.1 & 3.5 & 0.04 & Stable \\
\hline
\end{tabular}




\begin{tabular}{|c|c|c|c|c|c|c|c|c|}
\hline \multirow{2}{*}{$\begin{array}{l}\text { Bridge No. } \\
\text { (fig. 2) }\end{array}$} & \multirow[b]{2}{*}{ Bridge name } & \multirow[b]{2}{*}{ Highway/road } & \multirow{2}{*}{$\begin{array}{l}\text { Location } \\
\text { (mile) }\end{array}$} & \multirow{2}{*}{$\begin{array}{l}\text { Soundings } \\
\text { (years) }\end{array}$} & \multicolumn{3}{|c|}{ Low bed elevations (feet) } & \multirow{2}{*}{$\begin{array}{c}\text { Stable/ } \\
\text { unstable }\end{array}$} \\
\hline & & & & & Minimum & Maximum & $\begin{array}{c}\text { Changel } \\
\text { 10-foot width }\end{array}$ & \\
\hline 654 & Slana River & Tok Cutoff Highway & 74.3 & $2002-11$ & $2,183.7$ & $2,186.4$ & 0.25 & Stable \\
\hline 655 & Slana Slough & Tok Cutoff Highway & 74.8 & 2002,2004 & $2,187.9$ & $2,190.3$ & 0.22 & Stable \\
\hline 663 & Tok River & Tok Cutoff Highway & 101.5 & $2002,2004-14$ & $1,933.4$ & $1,938.5$ & 0.25 & Stable \\
\hline 670 & Kasilof River & Sterling Highway & 71.2 & $\begin{array}{l}2002,2004,2005 \\
\quad 2007-16\end{array}$ & 29.0 & 32.1 & 0.14 & Stable \\
\hline 671 & Kenai River & Sterling Highway & 58.2 & $2001-04$ & 40.9 & 42.7 & 0.11 & Stable \\
\hline 694 & Nenana River at McKinley & Parks Highway & 195.5 & $2004-11,2015$ & $1,784.6$ & $1,786.2$ & 0.08 & Stable \\
\hline 735 & Eagle River & Glacier Highway & 26.5 & 2006-08, 2010, 2011 & 11.9 & 13.2 & 0.09 & Stable \\
\hline 742 & Chilkat River & Haines Highway & 23.2 & $\begin{array}{l}2001,2004-11, \\
2013-16\end{array}$ & 108.9 & 116.2 & 0.17 & Stable \\
\hline 857 & Nenana River at Healy & Healy Road & 3.2 & $\begin{array}{l}1999,2000,2003-09 \\
2011,2015\end{array}$ & $1,239.1$ & $1,250.7$ & 0.28 & Stable \\
\hline 983 & Red Cloud River & $\begin{array}{l}\text { Anton Larson Bay } \\
\text { Road }\end{array}$ & 7.5 & $2005-11,2013-16$ & ${ }^{1} 86.5$ & 90.3 & 1.00 & Unstable \\
\hline 987 & Buskin River & $\begin{array}{l}\text { Anton Larson Bay } \\
\text { Road }\end{array}$ & 0.6 & 2006-09, 2011, 2013 & 23.1 & 23.9 & 0.10 & Stable \\
\hline 999 & Glacier Creek & Alyeska Road & 2.3 & $\begin{array}{l}1999,2004,2005, \\
2007-16\end{array}$ & 99.8 & 103.6 & 0.21 & Stable \\
\hline 1020 & Quartz Creek & Quartz Creek Road & 0.7 & $2012,2014-16$ & ${ }^{1} 86.8$ & 90.8 & 0.50 & Unstable \\
\hline 1021 & Crescent Creek & Quartz Creek Road & 2.6 & 2014,2016 & 89.6 & 90.3 & 0.08 & Stable \\
\hline 1127 & Safety Sound Estuary & Nome-Taylor Road & 20.8 & $\begin{array}{l}2007,2008,2010, \\
\quad 2011\end{array}$ & -26.0 & -24.0 & 0.03 & Stable \\
\hline 1147 & $\begin{array}{l}\text { Nenana River at Park } \\
\text { Station }\end{array}$ & Parks Highway & 202.4 & 1998, 2002 & $1,504.5$ & $1,505.8$ & 0.03 & Stable \\
\hline 1149 & Kenai River & Kenai River Crossing & 2.6 & 2009,2012 & -16.3 & -16.3 & 0.00 & Stable \\
\hline
\end{tabular}




\begin{tabular}{|c|c|c|c|c|c|c|c|c|}
\hline \multirow{2}{*}{$\begin{array}{l}\text { Bridge No. } \\
\text { (fig. 2) }\end{array}$} & \multirow[b]{2}{*}{ Bridge name } & \multirow[b]{2}{*}{ Highway/road } & \multirow{2}{*}{$\begin{array}{l}\text { Location } \\
\text { (mile) }\end{array}$} & \multirow{2}{*}{$\begin{array}{l}\text { Soundings } \\
\text { (years) }\end{array}$} & \multicolumn{3}{|c|}{ Low bed elevations (feet) } & \multirow{2}{*}{$\begin{array}{c}\text { Stable/ } \\
\text { unstable }\end{array}$} \\
\hline & & & & & Minimum & Maximum & $\begin{array}{c}\text { Change/ } \\
\text { 10-foot width }\end{array}$ & \\
\hline 1187 & Copper River Delta & $\begin{array}{c}\text { Copper River } \\
\text { Highway }\end{array}$ & 26.9 & $2005-16$ & -14.2 & -3.1 & 0.12 & Stable \\
\hline 1191 & Chena River at Pegar & Pegar Road & 0.5 & $2005-09$ & 407.3 & 408.0 & 0.04 & Stable \\
\hline 1200 & Independence Creek & Sterling/Ophir road & 33.1 & $2007,2011,2015$ & 191.6 & 93.1 & 0.71 & Unstable \\
\hline 1212 & Chena River at Nordale & Nordale Road & 2.3 & $2005-09$ & 432.7 & 436.4 & 0.29 & Stable \\
\hline 1235 & Four Mile Creek & $\begin{array}{l}\text { Williamsport/Pile } \\
\text { Road }\end{array}$ & 4.9 & $2007,2011,2015$ & 193.0 & 94.7 & 0.61 & Unstable \\
\hline 1243 & Nenana River at Windy & Parks Highway & 180.2 & $2007-16$ & $1,997.5$ & $1,999.4$ & 0.07 & Stable \\
\hline 1274 & Monashka Creek & Pillar Creek Road & 7.2 & 2011 & 1.1 & 1.3 & 0.08 & Stable \\
\hline 1321 & Timberline Creek & $\begin{array}{l}\text { Williamsport/Pile } \\
\text { Road }\end{array}$ & 8.5 & $\begin{array}{l}2007,2009,2011 \\
\quad 2015\end{array}$ & -8.4 & -6.9 & 0.13 & Stable \\
\hline 1383 & Lowe River & Richardson Highway & 19.3 & 2005-2008, 2010-16 & 386.5 & 389.8 & 0.17 & Stable \\
\hline 1384 & Lowe River & Richardson Highway & 19.4 & 2006-09, 2012 & 392.2 & 394.6 & 0.08 & Stable \\
\hline
\end{tabular}

${ }^{1}$ No as-built available, so reference is 100 . 
To assess streambed scour, Arneson and others (2012) recommend that a general assessment of stream stability, aggradation, or degradation follow guidelines in Lagasse and others (2012). The guidelines for assessment include evaluating (1) lateral and vertical stream stability factors, (2) flood frequency, (3) bank and bed material, (4) hydraulic conditions, (5) watershed sediment, (6) armoring potential, (7) rating curve, (8) and scour conditions. Streams in Alaska commonly tend to be naturally unstable because of high gradients. large sediment supply, lack of containment, or relatively frequent overbank floods. Human activity has a substantial effect on stream stability owing to dredging, in-stream mining, and erosion control. These factors may influence the vulnerability of structures and embankments to scour and erosion.

Only the minimum bed elevations are compared between surveys because the channel soundings were not always collected at the same distance interval along the length of the bridge. The average change in minimum bed elevation between successive soundings provides evidence of channel aggradation or degradation. The maximum change from the highest and the lowest minimum bed elevation across all the measured surveys is used to determine relative stream stability (fig. 3). Elevation changes were normalized by the bridge width because vertical changes in a 200 -ft-wide river are expected to be greater than in a 20 -ft-wide stream. Sites with less than $\pm 0.40 \mathrm{ft}$ of relative change per $10 \mathrm{ft}$ of channel width between surveys were considered stable and sites that had greater than or equal to $\pm 0.40 \mathrm{ft}$ of change per $10 \mathrm{ft}$ of channel width were considered unstable (Beebee and others, 2017). Tabular data for all cross sections collected for this study are presented in appendix 1 . Cross sections of bed elevation and bridge geometry are included in appendix 2, except for those sites discussed in detail in the following sections. 


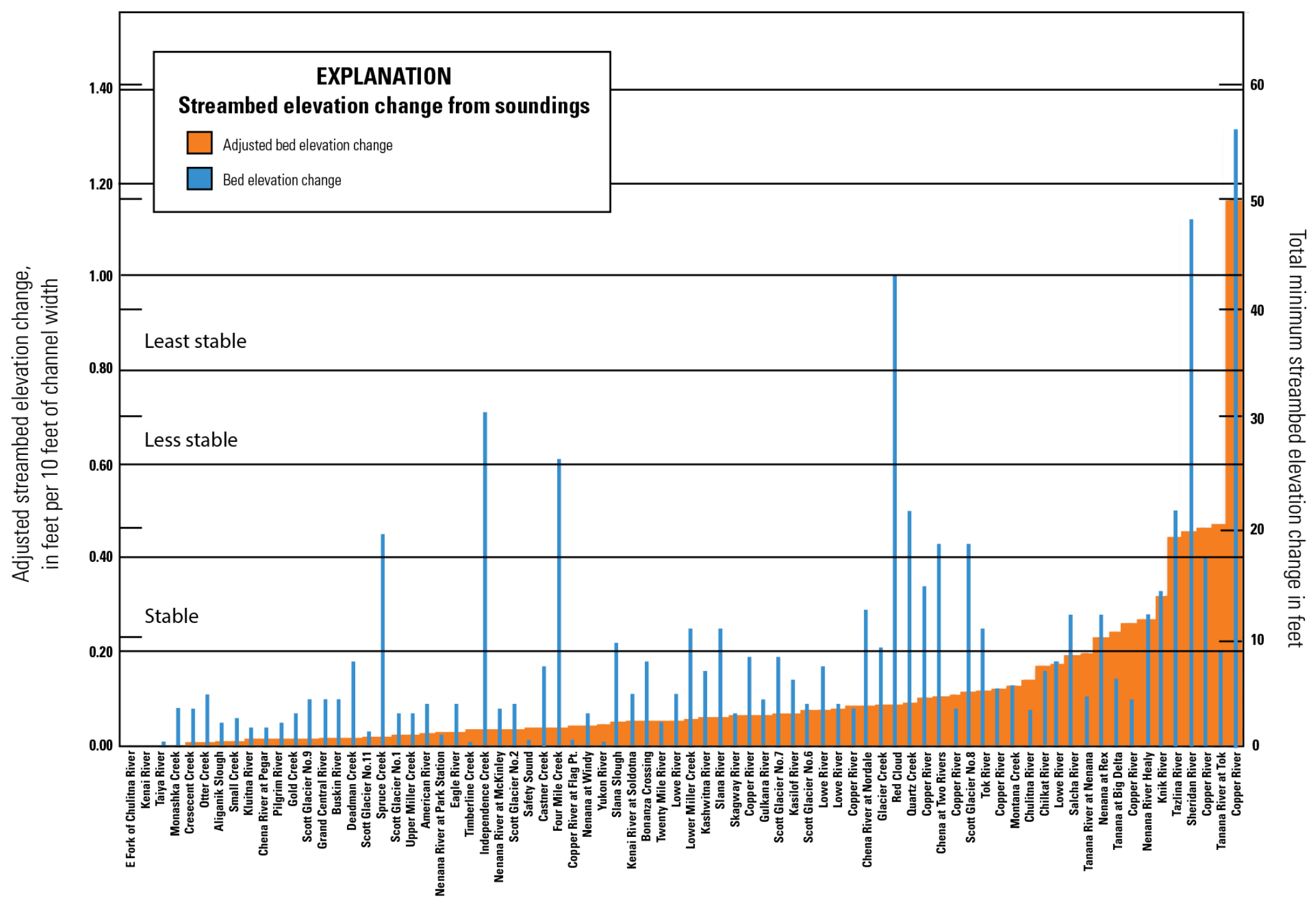

Figure 3. Graph showing sounding-based stream stability at 76 river- and stream-spanning bridges in Alaska. 


\section{Intensive Monitoring Sites}

These sites were part of the initial monitoring program following phase 2 (Conaway, 2004), are part of the current monitoring program (Beebee and others, 2017), or were previously part of the monitoring program and have a minimum of 9 years of soundings data. Channel soundings were either consistently measured on the upstream or downstream side of the bridge, but in many cases both upstream and downstream were measured because of the variations in data collection methods. Many of these sites also had a pier-mounted sonar on one or more of the piers over some period. The purpose of the sonars was to monitor real-time bed elevation and provide a nearly continuous record of bed elevation responses to discharge and sediment supply. The sites that have sonar data associated with the cross sections are indicated by an asterisk (*) next to the name in the following sections. Gaps in the plots of cross-sectional data are the result of large woody debris that prevented complete channel soundings. Owing to the tendency for Alaska rivers and creeks to be naturally unstable, the streambanks typically are heavily riprapped through bridges unless noted otherwise. Because of discrepancies in funding from site to site, varying degrees of data were collected both annually and over the long term.

\section{Bridge 202, Tanana River at Nenana*}

Tanana River is a glacially fed river with headwaters in the Alaska Range. The river crossing at Nenana is hydraulically complex owing to the confluence of the Nenana River downstream of the bridge and the division of the main channel by an island into a main flow and an adjacent slough (Langley, 2006). The main pier on the bridge also is oriented about 15 degrees to flow. The river is relatively straight for many channel widths upstream of the bridge. Cross sections were collected along the upstream side of the bridge during 1998 and 2003-16 (figs. 4-7). Although the soundings indicate a relatively stable streambed across most of the channel, maximum observed scour around the pier was 8 $\mathrm{ft}$, and maximum aggradation along the left bank also was $8 \mathrm{ft}$. 
BN 202 Tanana River at Nenana, upstream

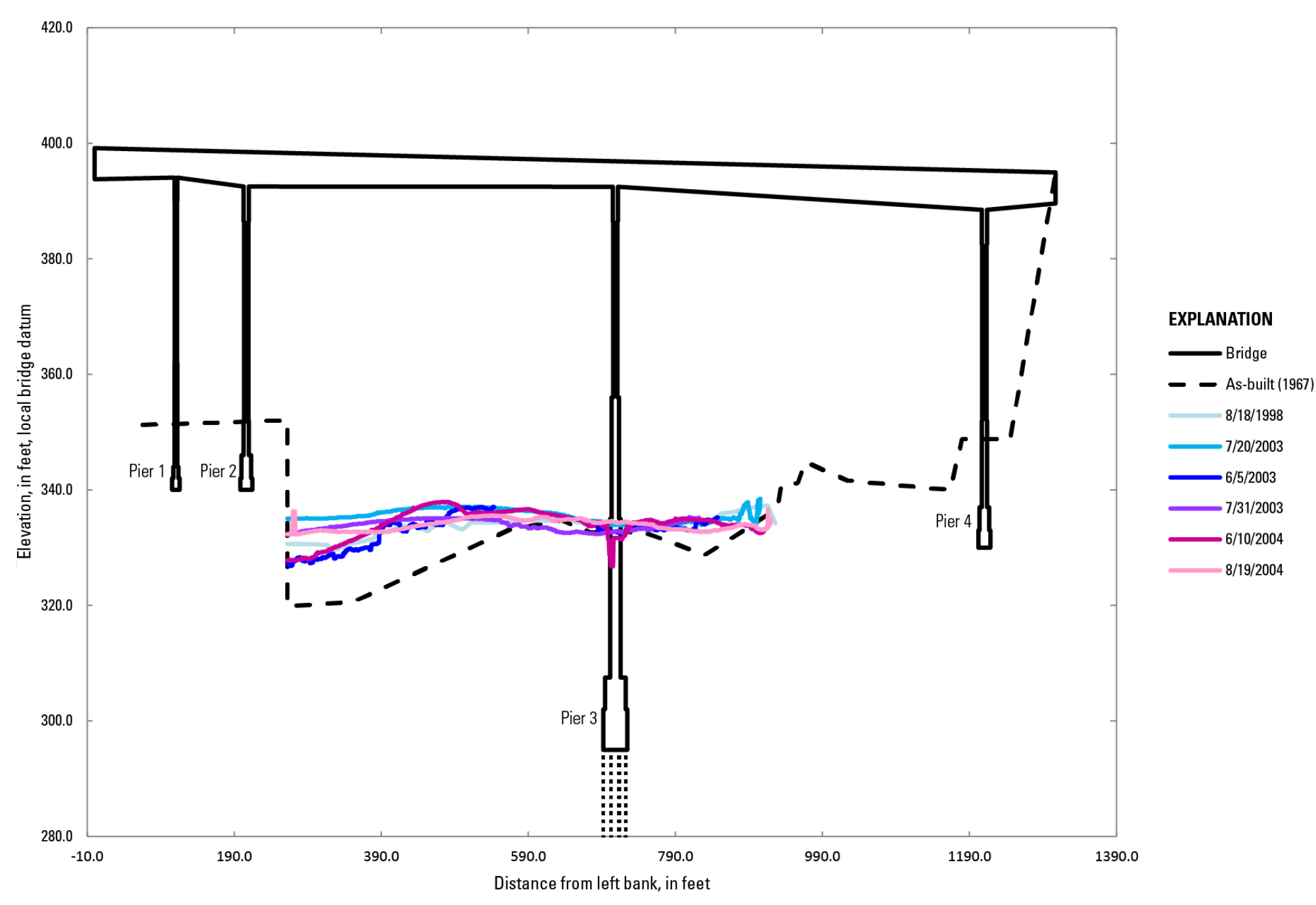

Figure 4. Cross sections showing upstream soundings at bridge 202, Tanana River at Nenana, Alaska, 1998-2004. 


\section{BN 202 Tanana River at Nenana, upstream}

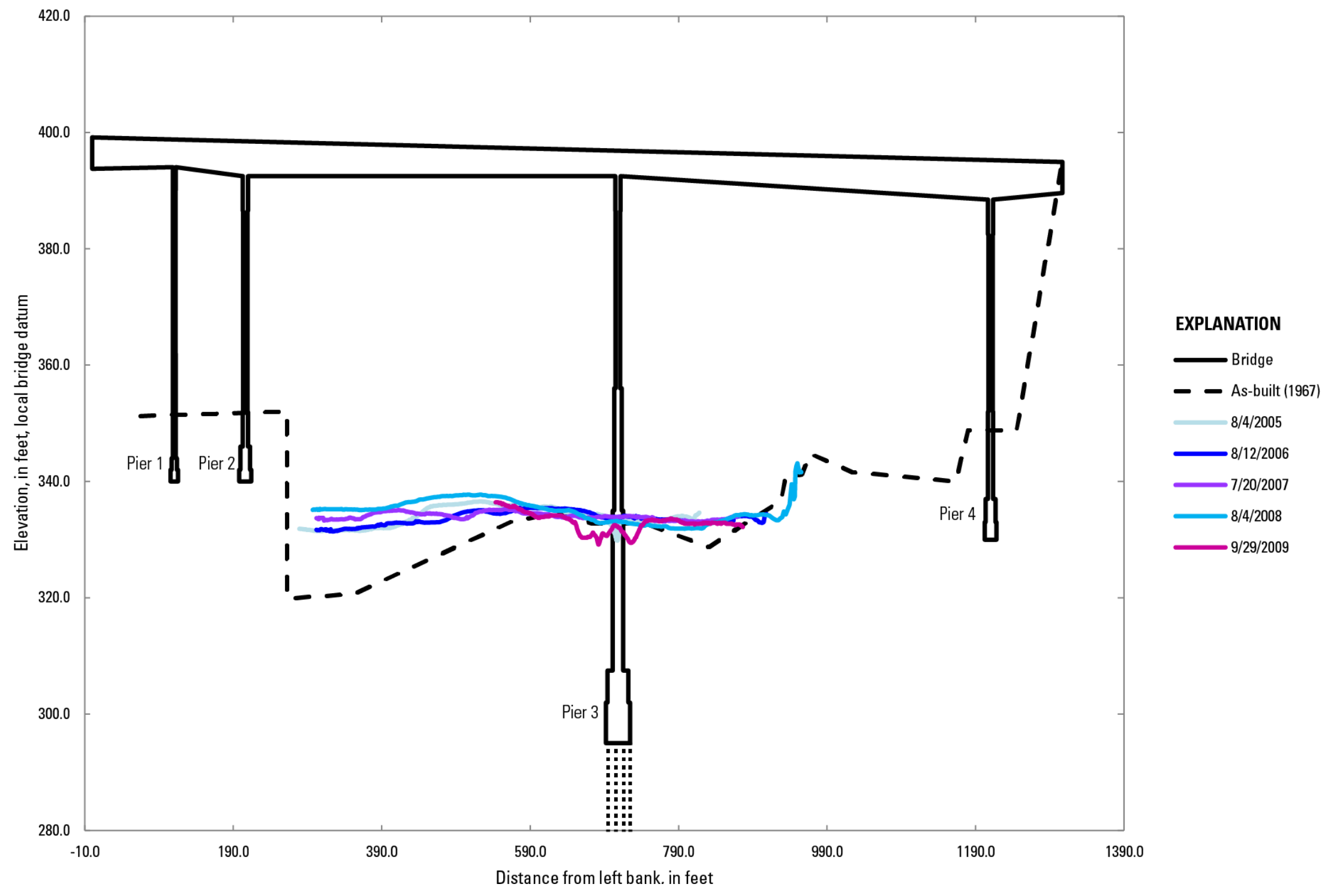

Figure 5. Cross sections showing upstream soundings at bridge 202, Tanana River at Nenana, Alaska, 2005-09. 
BN 202 Tanana River at Nenana, upstream

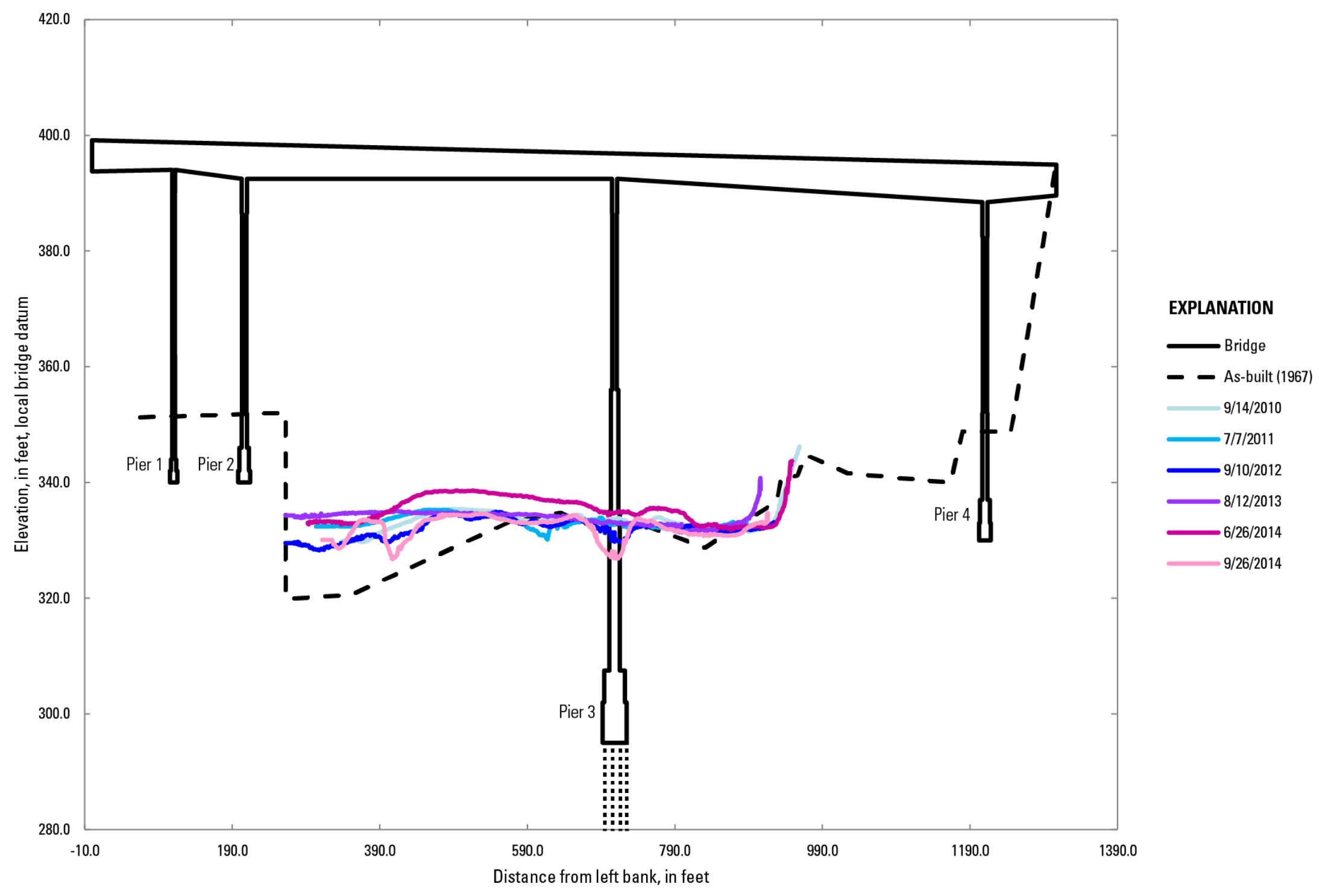

Figure 6. Cross sections showing upstream soundings at bridge number 202, Tanana River at Nenana, Alaska, 2010-14. 
BN 202 Tanana River at Nenana, upstream

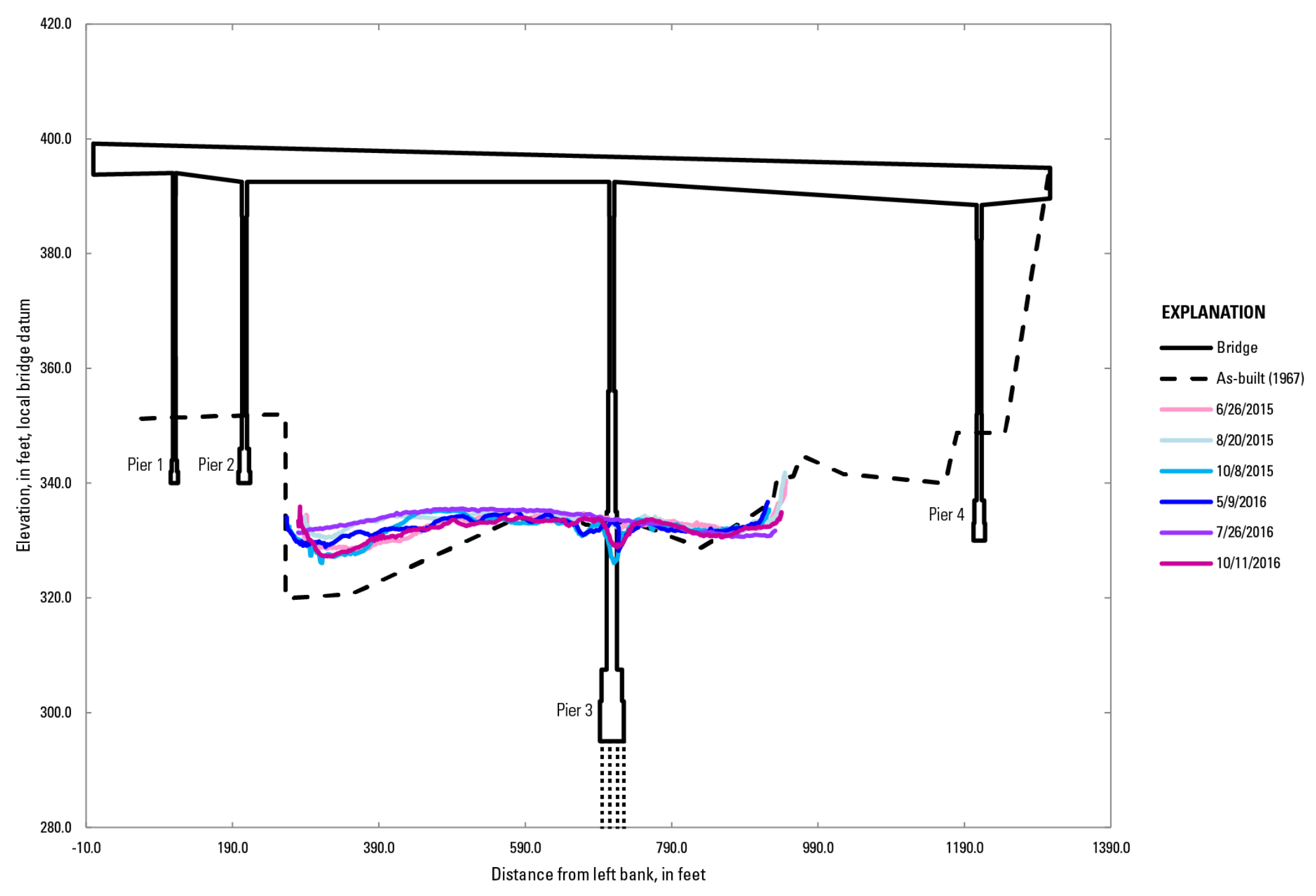

Figure 7. Cross section showing upstream soundings at bridge 202, Tanana River at Nenana, Alaska, 2015-16 


\section{Bridge 212, Kashwitna River*}

Kashwitna River a glacially fed river draining the Talkeetna Mountains. It is a single strand meandering river for most of its length. At low-to-moderate stages, flow is concentrated on the left edge of the channel through the bridge reach and a gravel bar is exposed about $350 \mathrm{ft}$ downstream of the bridge. At higher stages, the gravel bar is mostly submerged and covered with logs and large debris. Logs and debris also tend to accumulate on the upstream side of the piers and prevented complete crosssection surveys.

Cross sections are collected from a pedestrian bridge $30 \mathrm{ft}$ upstream of the highway bridge and are considered representative of the channel at the highway bridge owing to the proximity of the bridges and the uniformity of the channel between the bridges. Cross sections were collected from 2005 to 2016 (figs. 8-10) with a maximum streambed scour of $3 \mathrm{ft}$ observed in the left part of the channel. The remainder of the channel has aggraded $3 \mathrm{ft}$ since the as-built survey. Despite relatively consistent debris accumulation on the piers, localized pier scour is not evident. 
BN 212 Kashwitna River, upstream

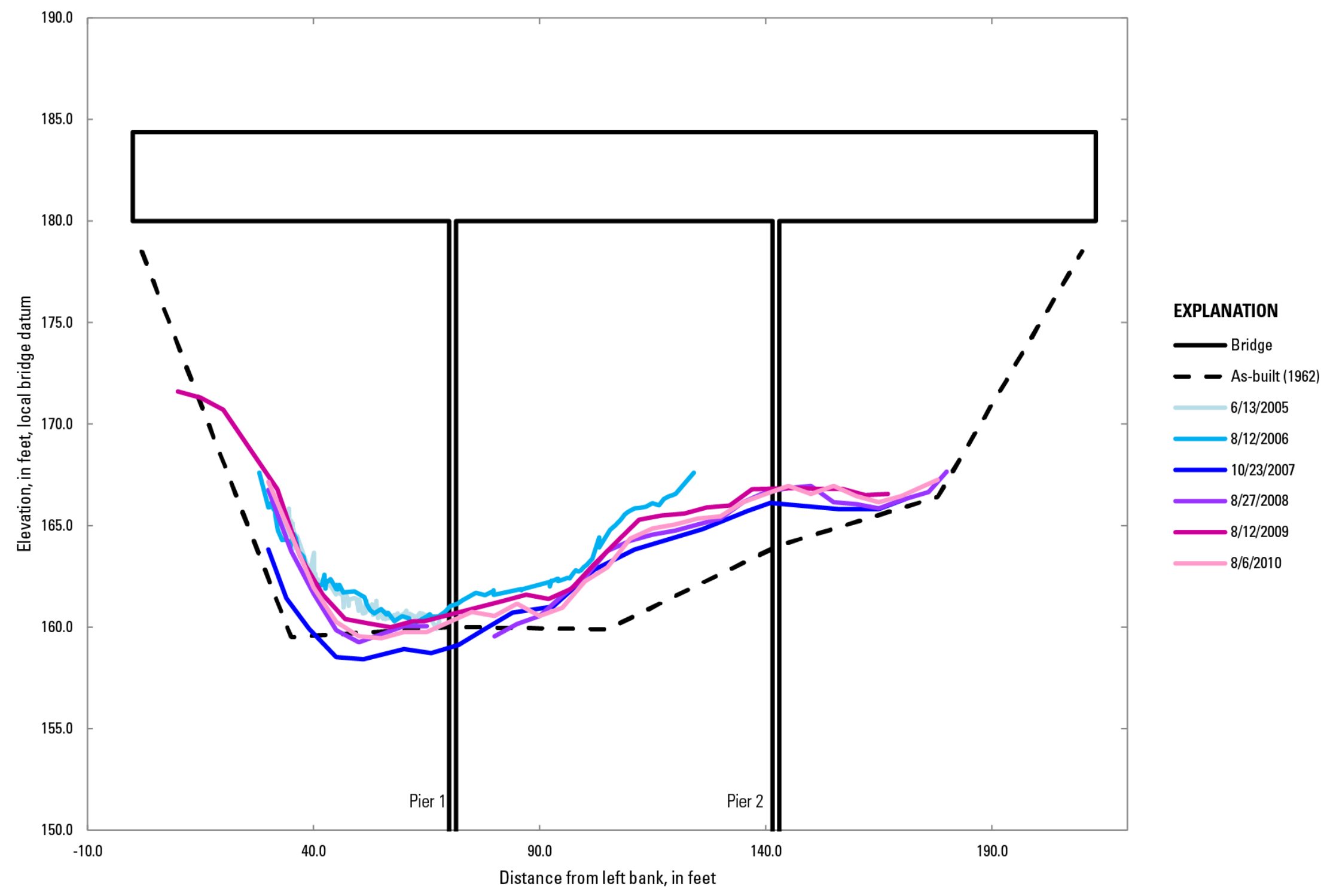

Figure 8. Cross sections showing upstream soundings at bridge 212, Kashwitna River, Alaska, 2005-10. 
BN 212 Kashwitna River, upstream

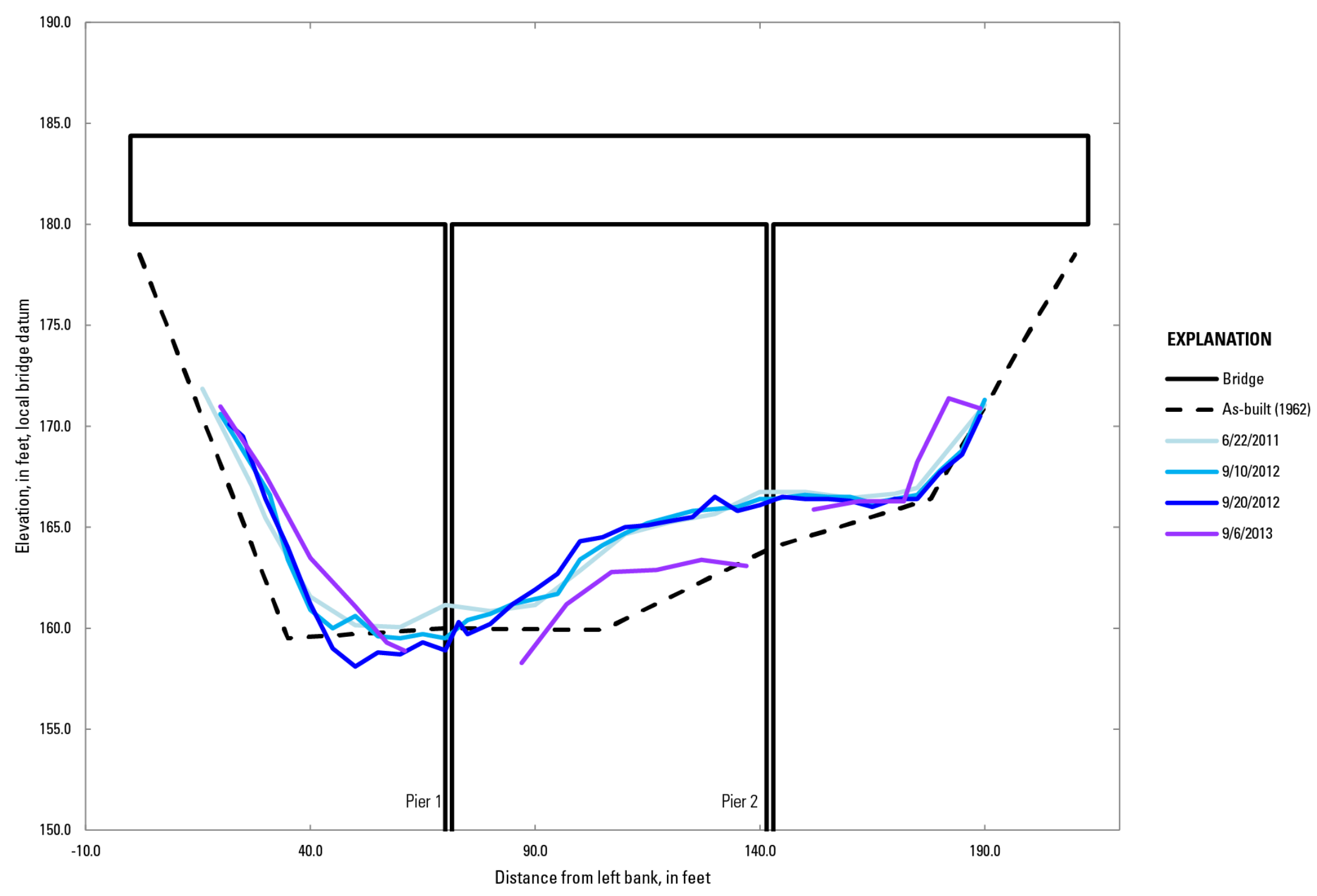

Figure 9. Cross sections showing upstream soundings at bridge 212, Kashwitna River, Alaska, 2011-13. 
BN 212 Kashwitna River, upstream

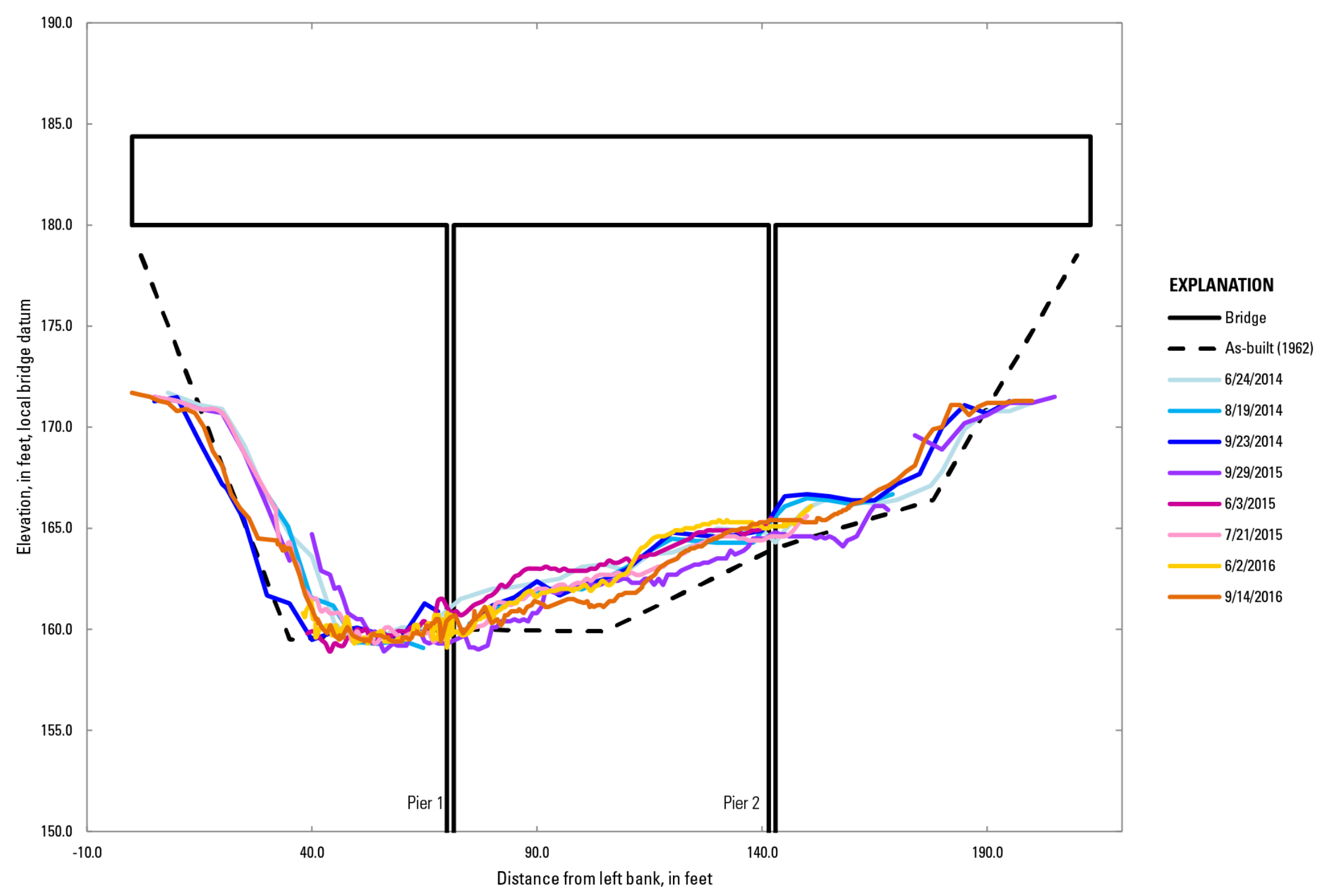

Figure 10. Cross sections showing upstream soundings at bridge 212, Kashwitna River, Alaska, 2014-16. 


\section{Bridge 215, Montana Creek*}

Montana Creek originates in the Talkeetna Range, but is not glacially fed. The channel curves right upstream of the bridge but is straight through the bridge reach. The channel constricts slightly at the highway bridge. Overbank flow is confined to the right side of the channel, which is vegetated with small to medium bushes and a few trees, whereas the left bank is armored in riprap. Both bridge piers are prone to debris accumulation. Cross sections were measured along the upstream side of the bridge from 2004 to 2016 (figs. 11-13). Channel elevations are stable in the center of the channel with less than $2 \mathrm{ft}$ of scour. Maximum local scour at the piers of as much as $5 \mathrm{ft}$ was observed, and channel width has increased $10 \mathrm{ft}$ along the right bank since the as-built survey. 
BN 215 Montana Creek, upstream

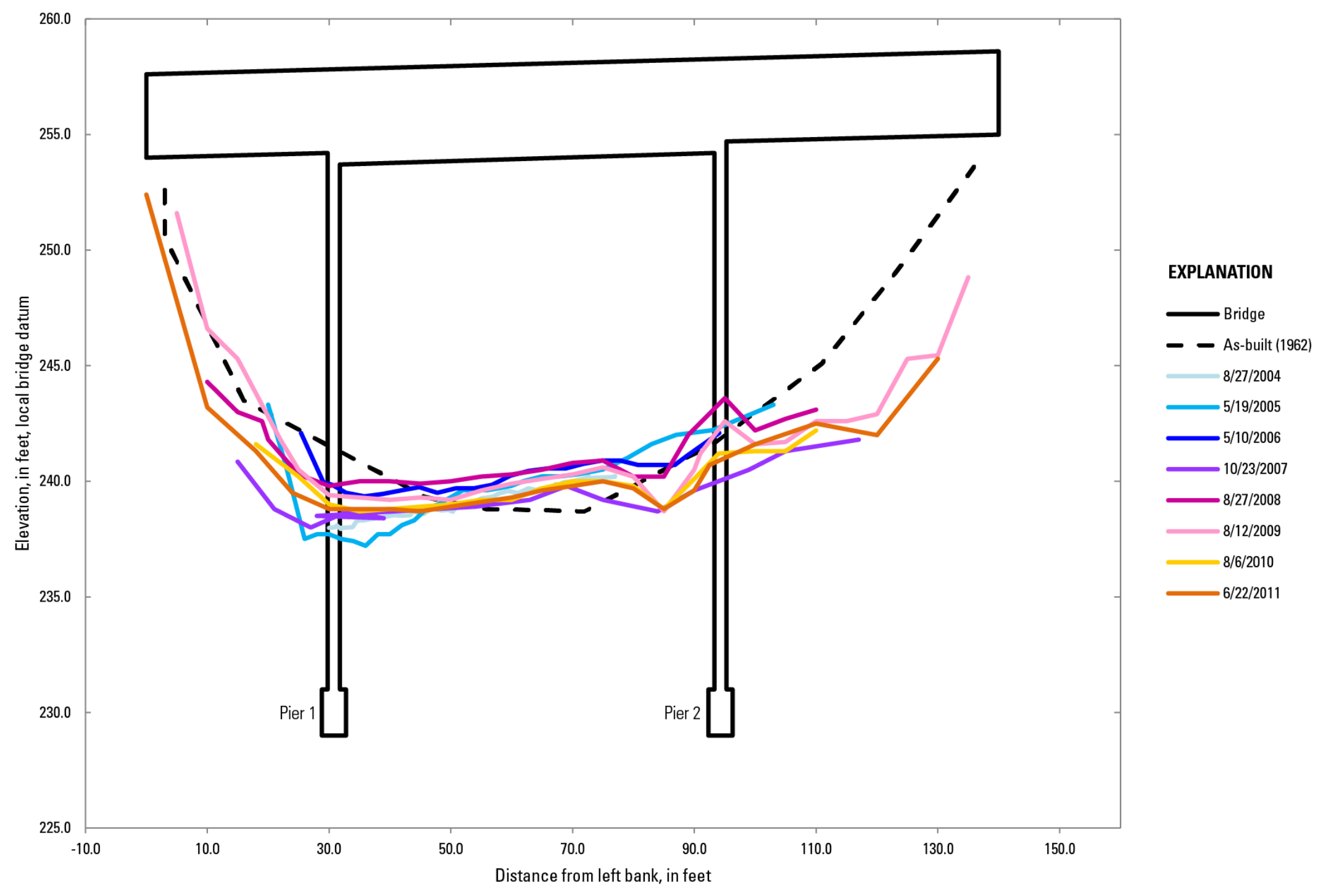

Figure 11. Cross sections showing upstream soundings at bridge 215, Montana Creek, Alaska, 2004-11. 


\section{BN 215 Montana Creek, upstream}

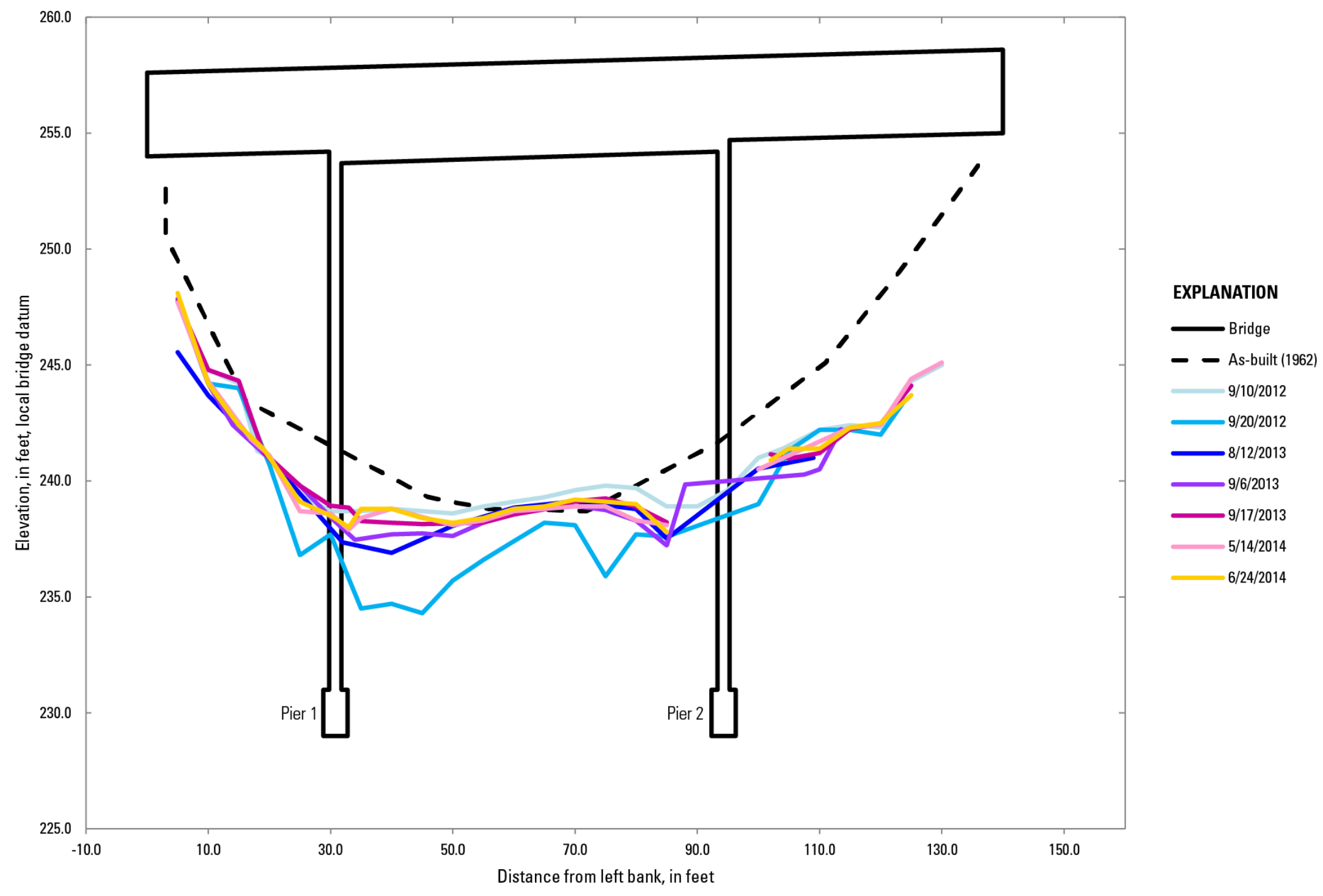

Figure 12. Cross sections showing upstream soundings at bridge 215, Montana Creek, Alaska, 2012-14. 
BN 215 Montana Creek, upstream

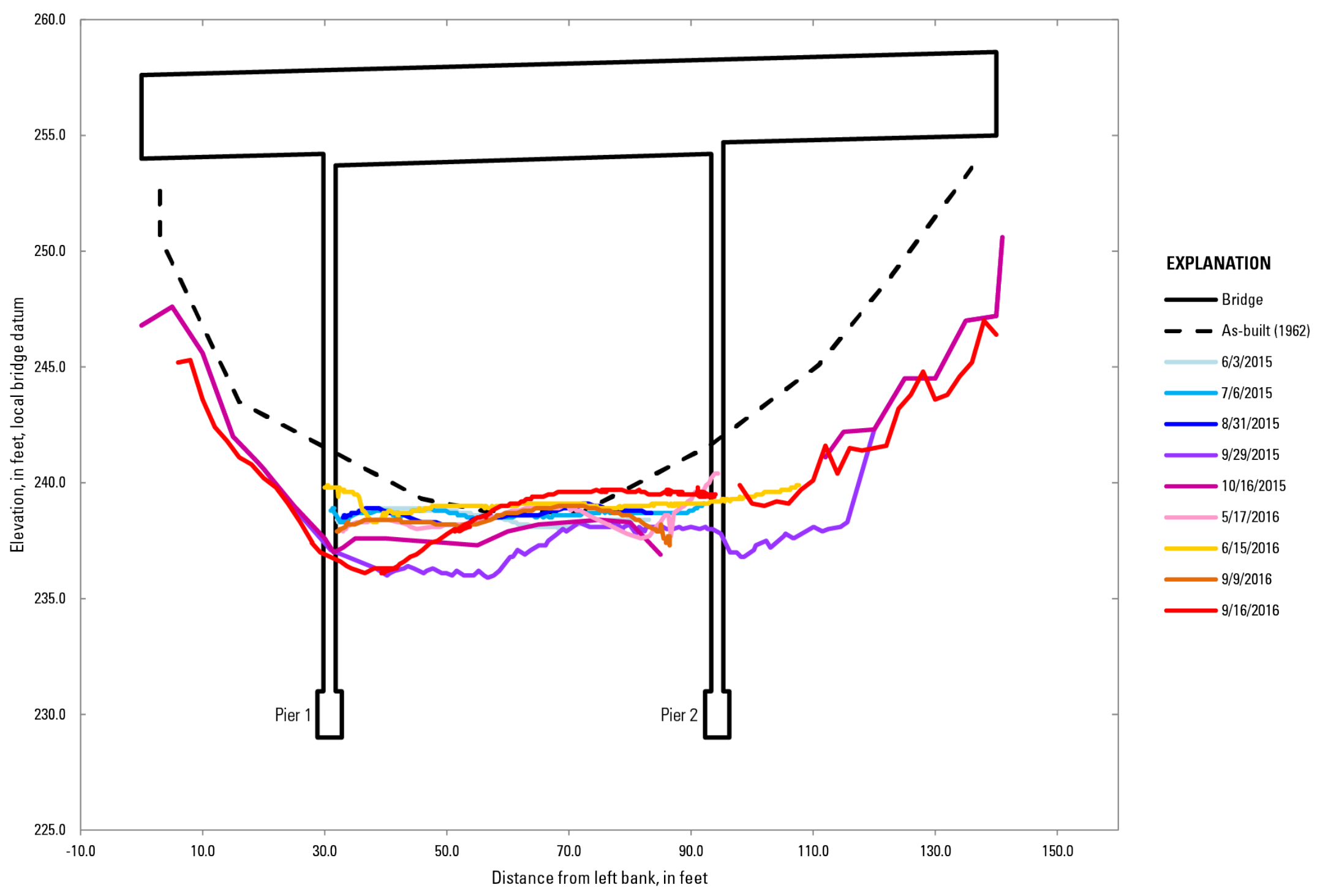

Figure 13. Cross sections showing upstream soundings at bridge 215, Montana Creek, Alaska, 2015-16. 


\section{Bridge 230, Sheridan River*}

Sheridan River originates in a proglacial lake in front of the Sheridan Glacier 3 mi upstream of the bridge. Heavy rainfall and rain on snow result in rapid increases in discharge at this site. Multiple discharges greater than the previously estimated 1-percent annual exceedance probability flow have occurred at this site over the course of this monitoring (2005-16) (Conaway, 2007). A riprap dike extends upstream of the bridge on the right bank to prevent overbank flow that has previously overtopped and closed the Copper River Highway on several occasions. Additionally, a guide bank directs flow into the bridge on the left bank. The channel is constricted by the bridge opening and approaches the bridge at a 20-35-degree angle. During floods, it is not uncommon for the road and low-lying areas around the bridge to be submerged under water. The bridge is prone to collecting large woody debris at the piers and between spans, which reduces the conveyance of flow. Cross-sectional surveys were done at the Sheridan River from 2004 to 2016 (figs. 14-17). Scour is concentrated at the two piers closest to the right bank. Maximum scour observed at these piers is $20 \mathrm{ft}$. Aggradation between the abutment and the left bank pier of about $3 \mathrm{ft}$ also has been observed. 
BN 230 Sheridan River, upstream

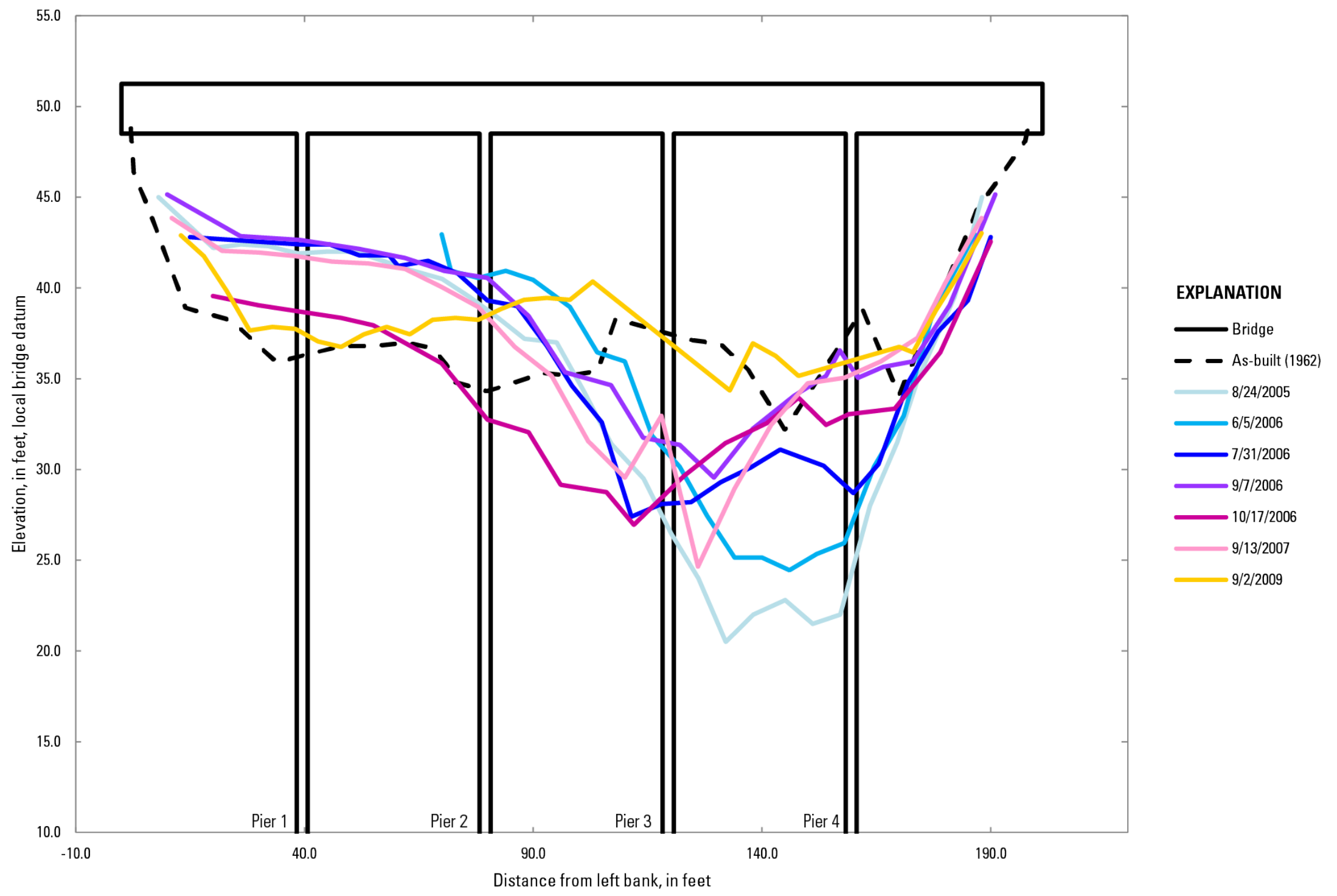

Figure 14. Cross sections showing upstream soundings at bridge 230, Sheridan River, Alaska, 2005-09. 


\section{BN 230 Sheridan River, upstream}

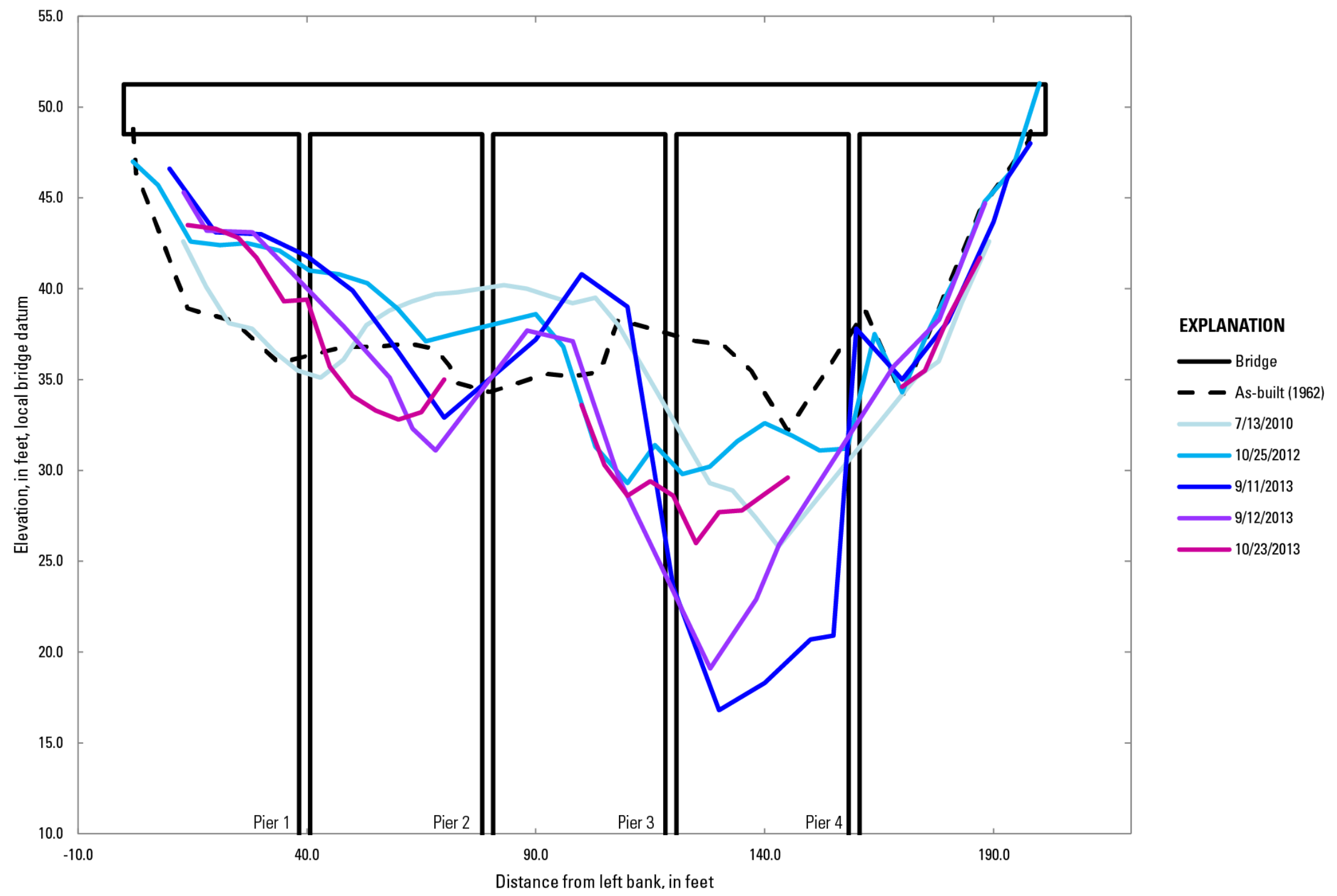

Figure 15. Cross sections showing upstream soundings at bridge 230, Sheridan River, Alaska, 2010-13. 
BN 230 Sheridan River, upstream

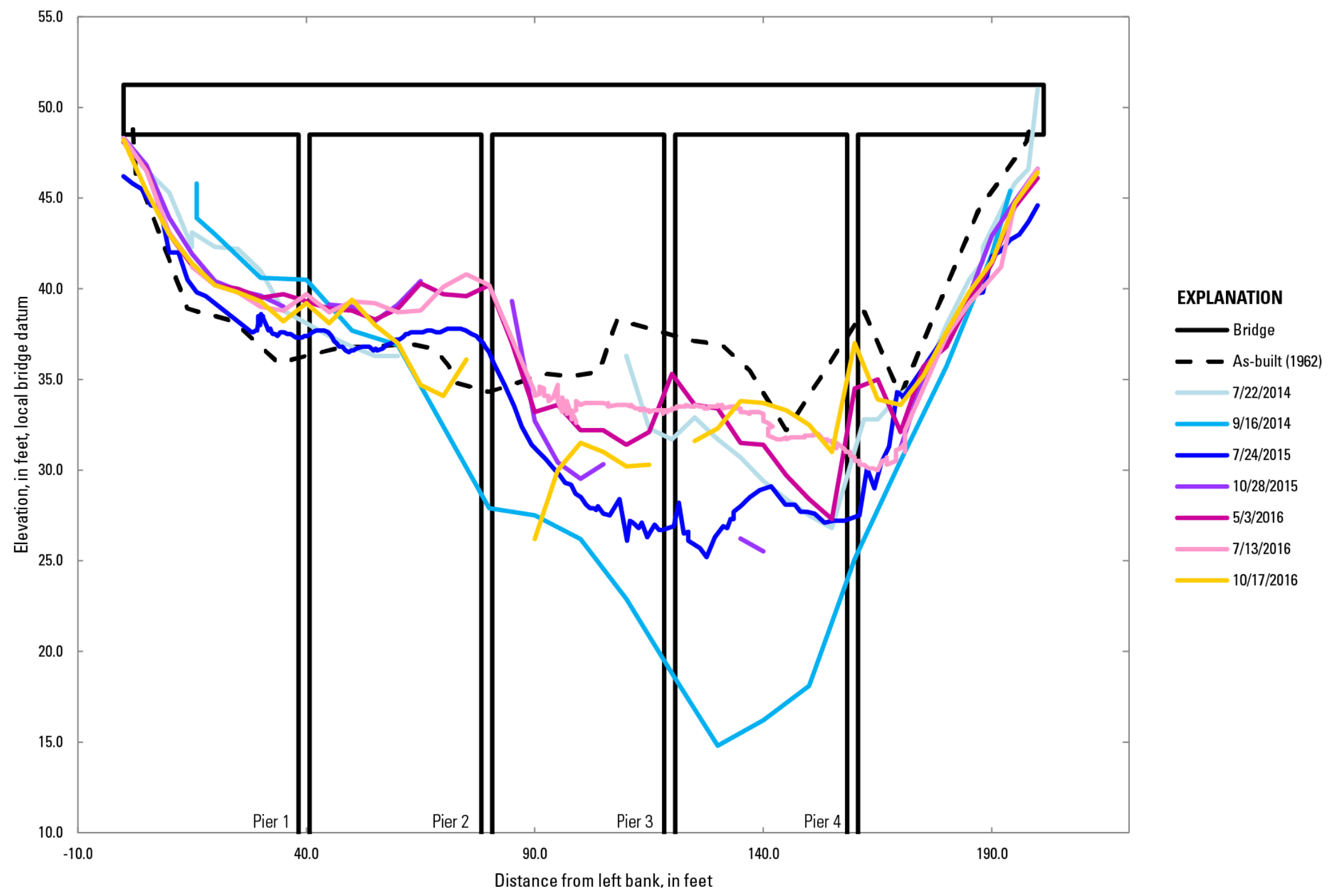

Figure 16. Cross sections showing upstream soundings at bridge 230, Sheridan River, Alaska, 2014-16. 
BN 230 Sheridan River, downstream

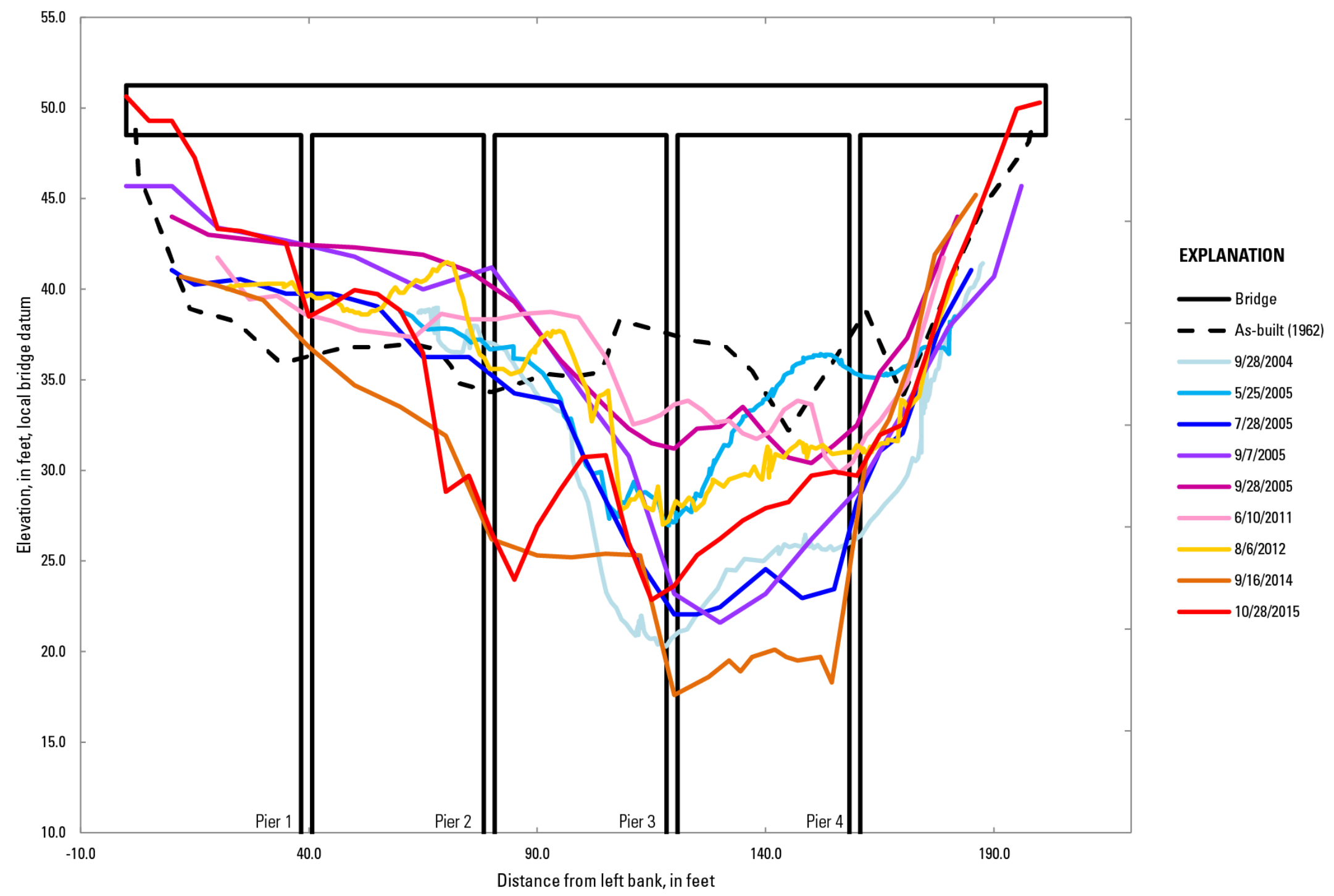

Figure 17. Cross sections showing downstream soundings at bridge 230, Sheridan River, Alaska, 2004-15. 
Bridges $331,332,333,334,336,339^{*}, 340^{*}, 342^{*}$, and $1187^{\star}$, Copper River Delta

The Copper River Basin is the sixth largest river basin in Alaska, with headwaters originating in multiple glaciated mountain ranges. The Copper River Highway crosses the Copper River Delta over 12 bridges, 9 of which have been monitored for scour to varying degrees (figs. 18-43). Large-scale channel migration across the Copper River Delta has changed flow distribution through the bridges several times since the highway was constructed (Conaway, 2007). In 2006, 15-24 percent of the Copper River flowed through bridge 339, and 53-81 percent flowed through bridge 342. By 2012, flow through bridge 339 increased to over 50 percent of the total flow of the Copper River and ultimately washed away the approach to the bridge. As of 2019, 84 percent of the Copper River still flows through bridge 342, bridge 339, and the former location of the highway between them. Most scour in the Copper River owes to redistribution of flow across the delta fan and the resultant concentration of flow through bridge openings. Brabets and Conaway (2009) detailed the complex dynamics of the Copper River Delta and the effect on the bridges. 
BN 331 Copper River Delta, downstream

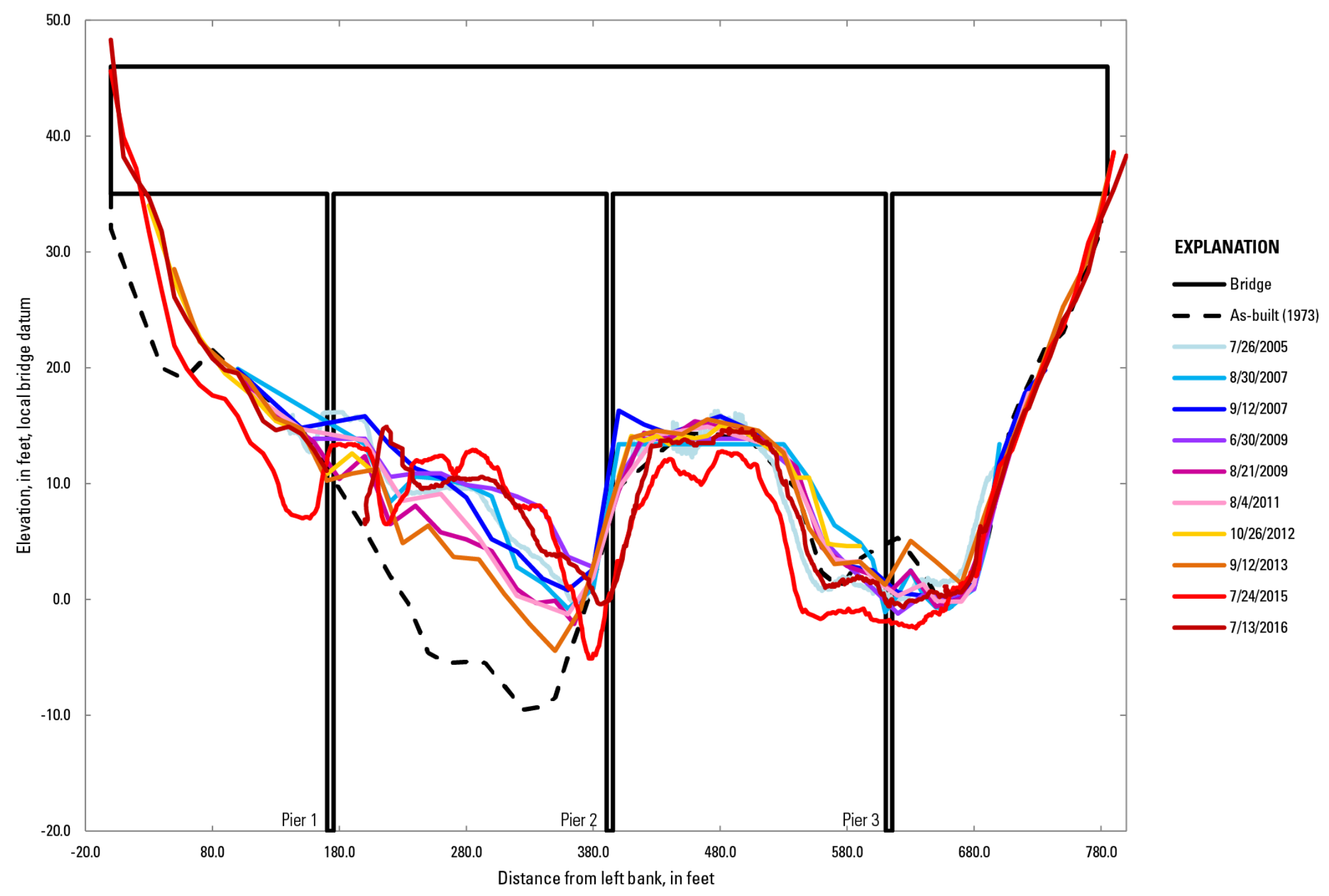

Figure 18. Cross sections showing downstream soundings at bridge 331, Copper River Delta, Alaska, 2005-16. 
BN 331 Copper River Delta, upstream

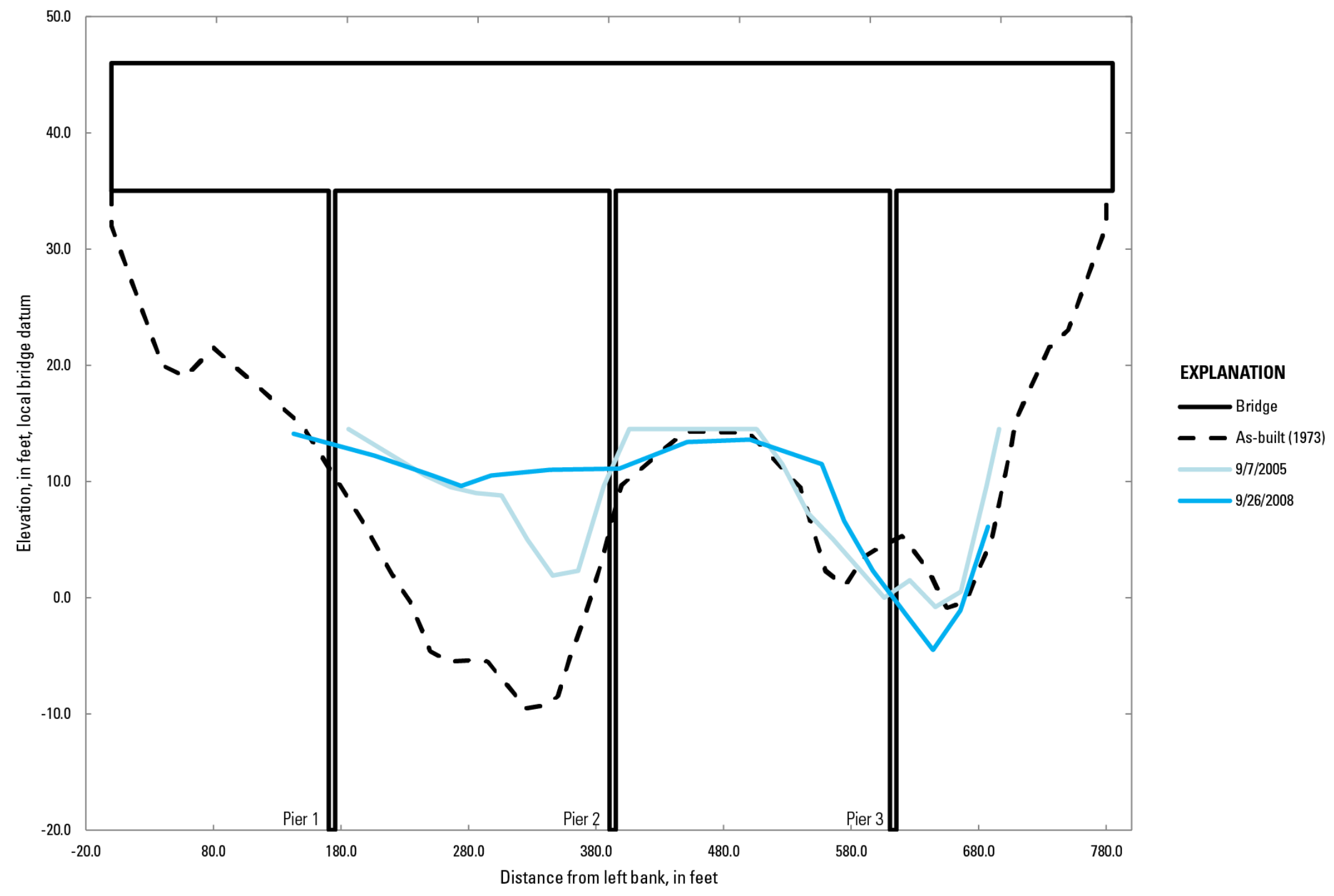

Figure 19. Cross sections showing upstream soundings at bridge 331, Copper River Delta, Alaska, 2005-08. 
BN 332 Copper River Delta, downstream

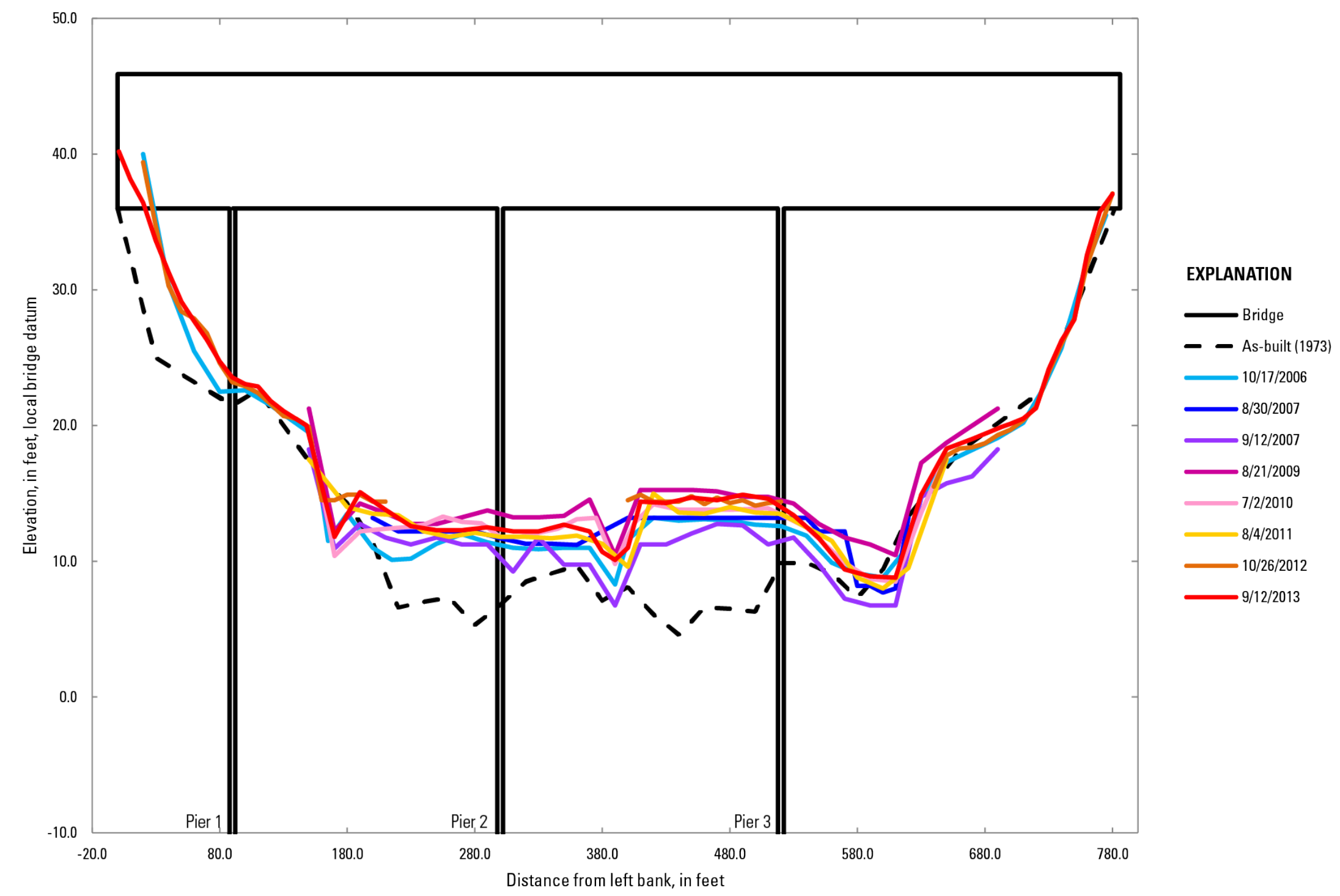

Figure 20. Cross sections showing downstream soundings at bridge 332, Copper River Delta, Alaska, 2006-13. 
BN 332 Copper River Delta, upstream

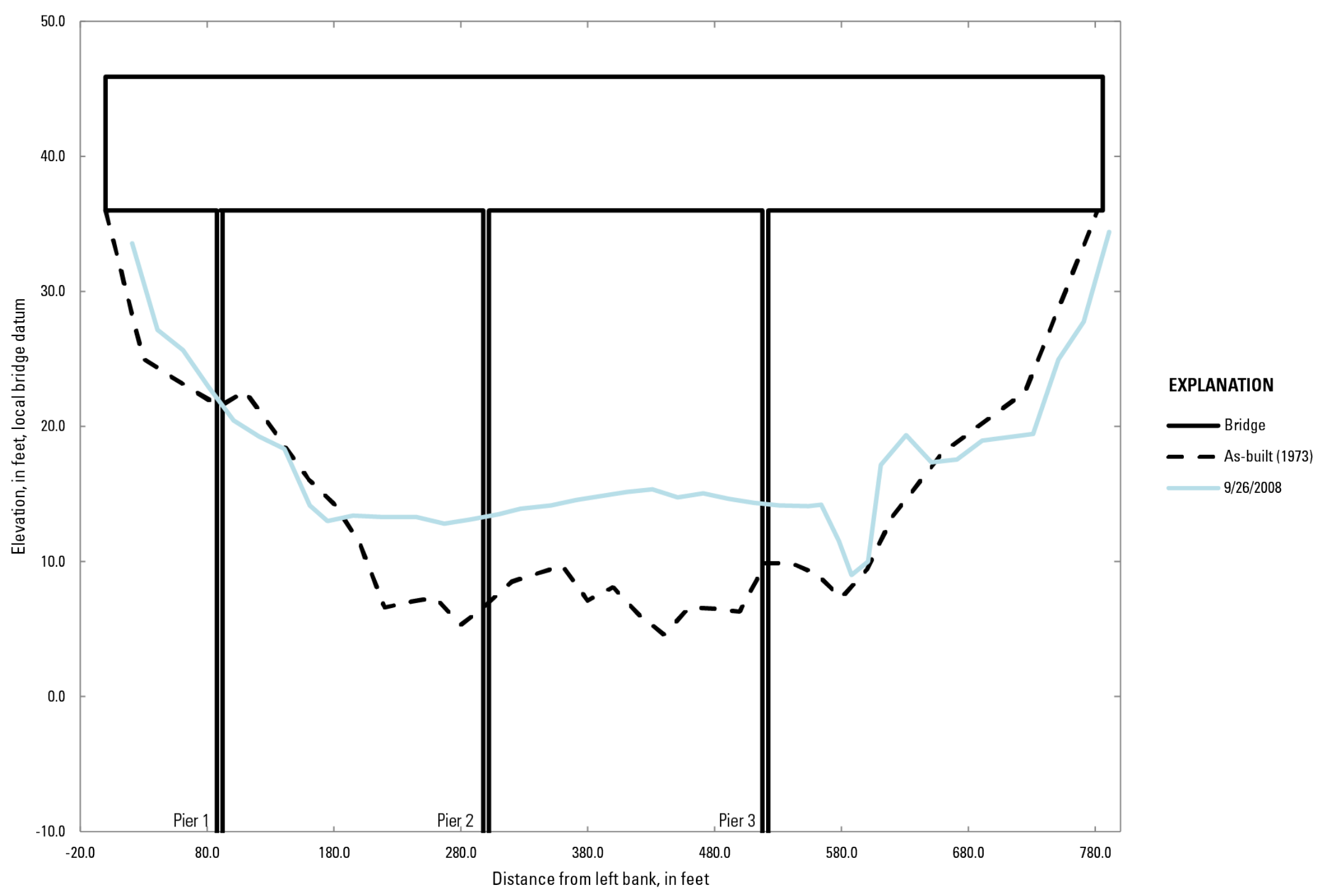

Figure 21. Cross sections showing upstream soundings at bridge 332, Copper River Delta, Alaska, 2008. 
BN 333 Copper River Delta, upstream

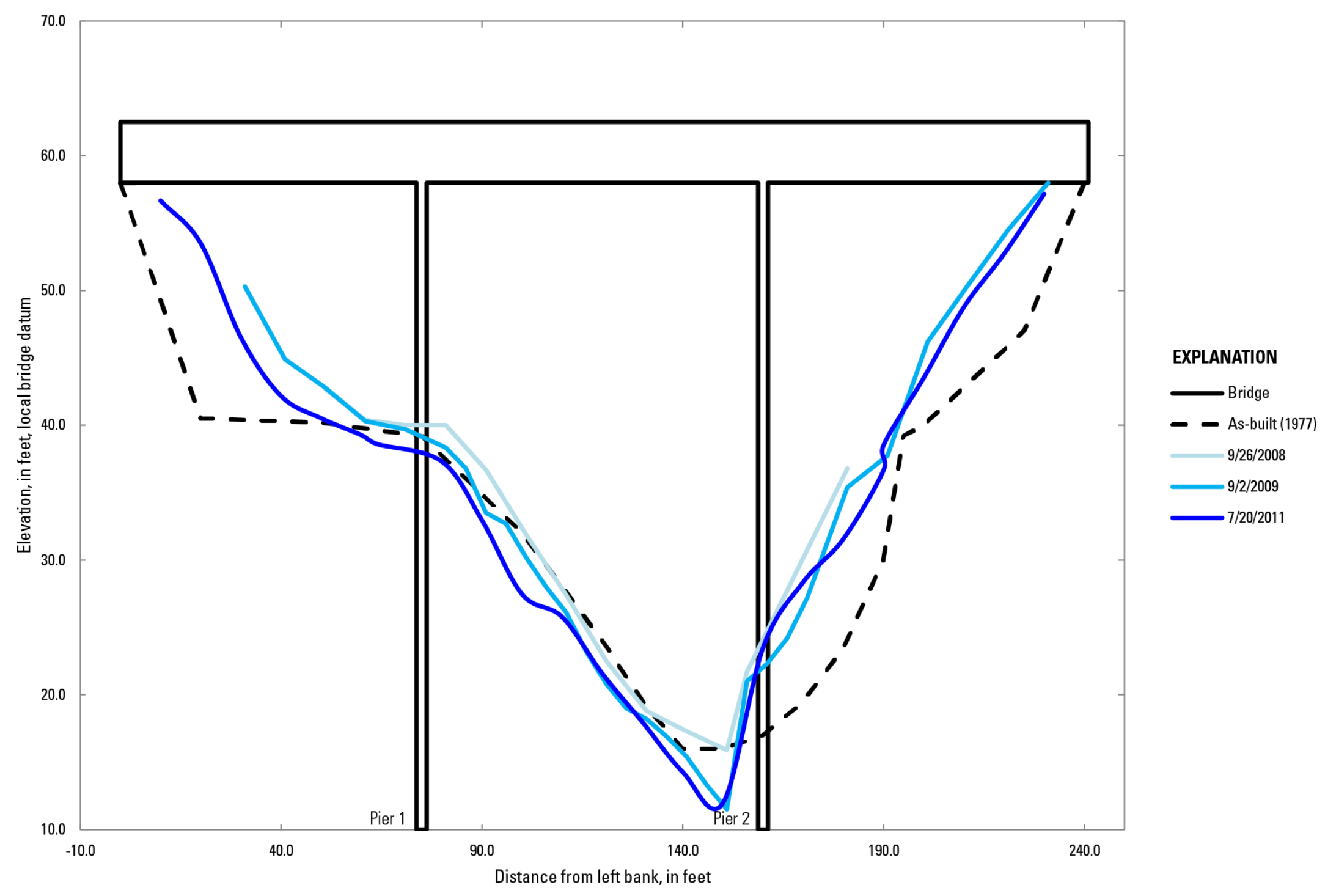

Figure 22. Cross sections showing upstream soundings at bridge 333, Copper River Delta, Alaska, 2008-11. 
BN 333 Copper River Delta, downstream

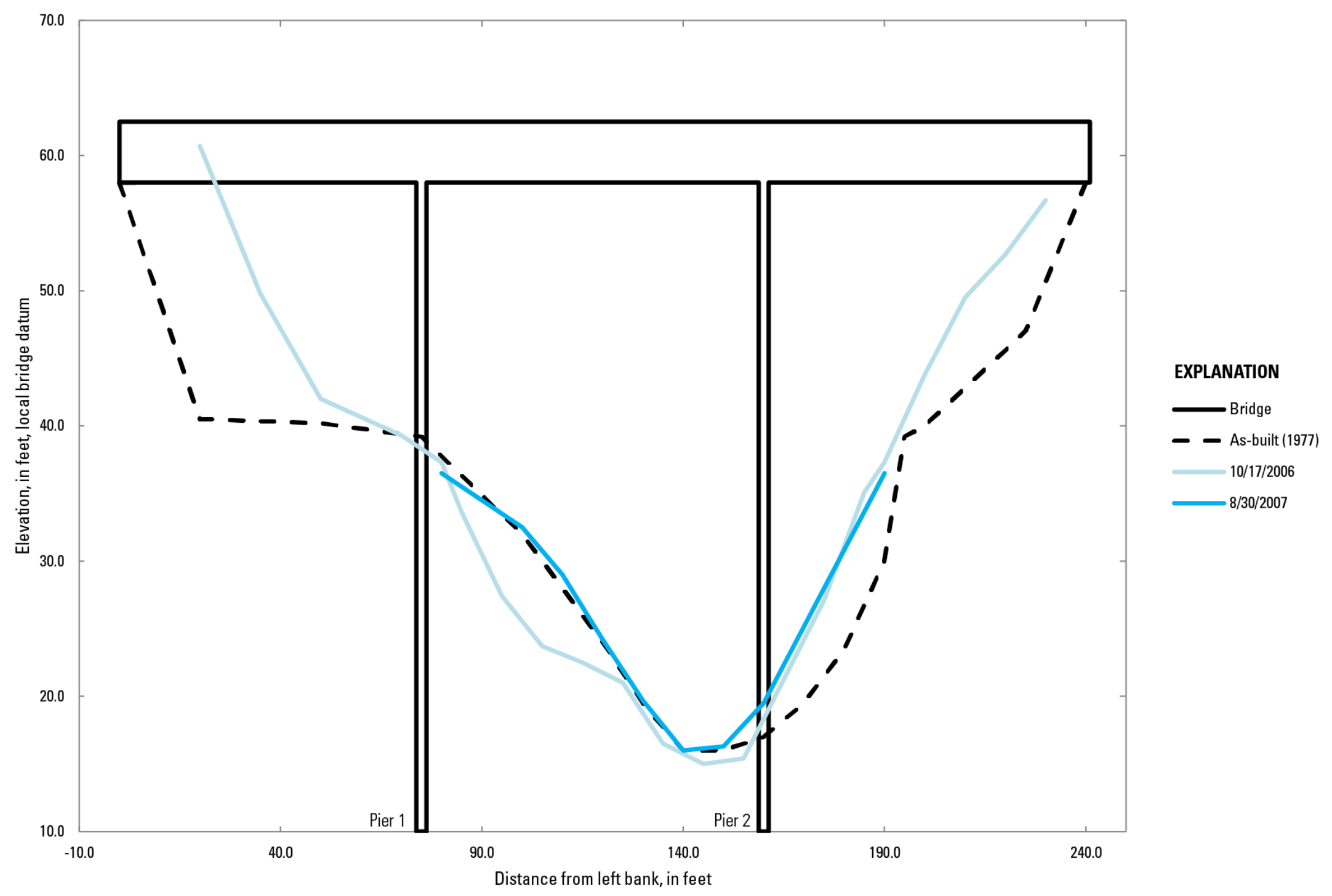

Figure 23. Cross sections showing downstream soundings at bridge 333, Copper River Delta, Alaska, 2006-07. 


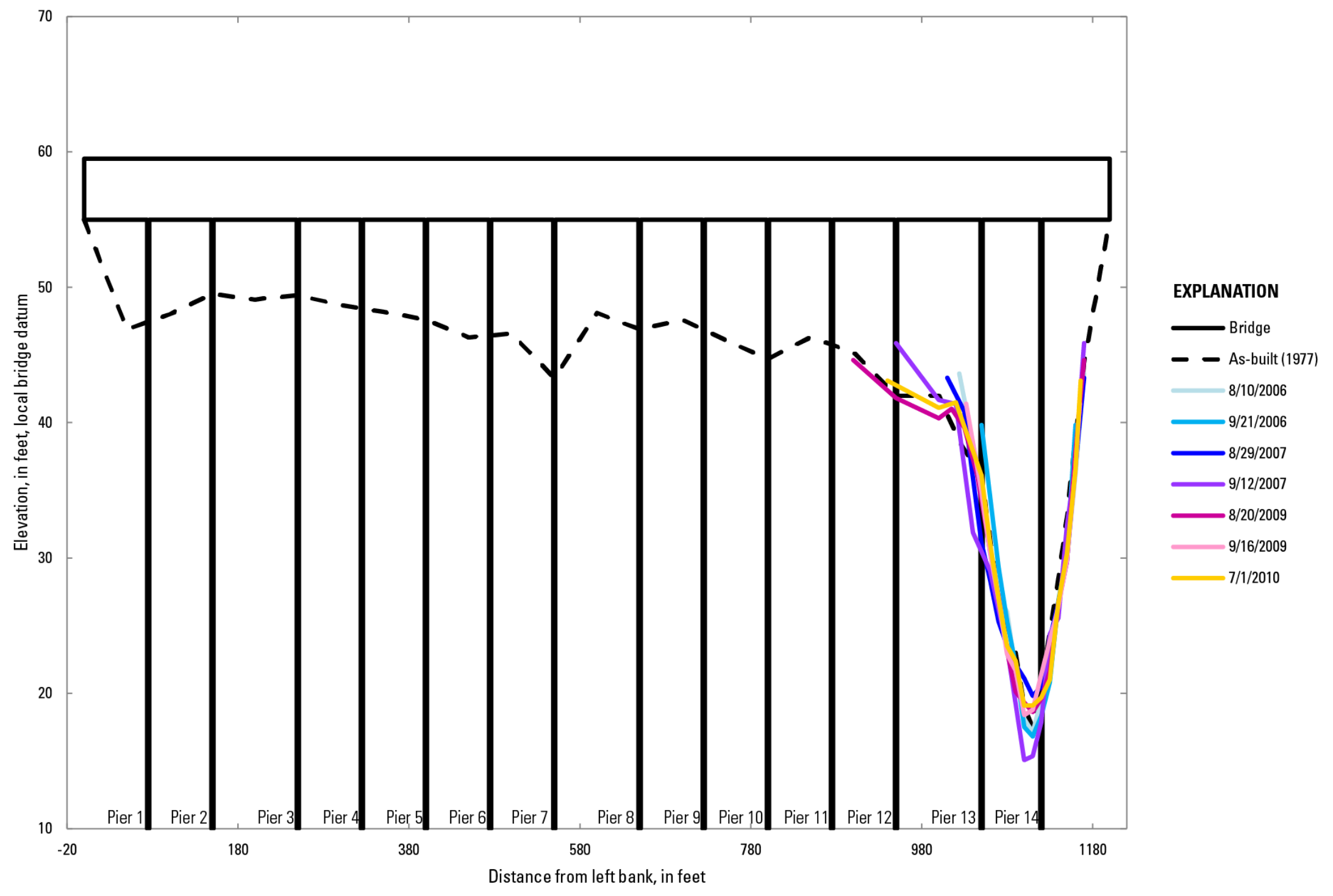

Figure 24. Cross sections showing downstream soundings at bridge 334, Copper River Delta, Alaska, 2006-10. 
BN 334 Copper River Delta, upstream

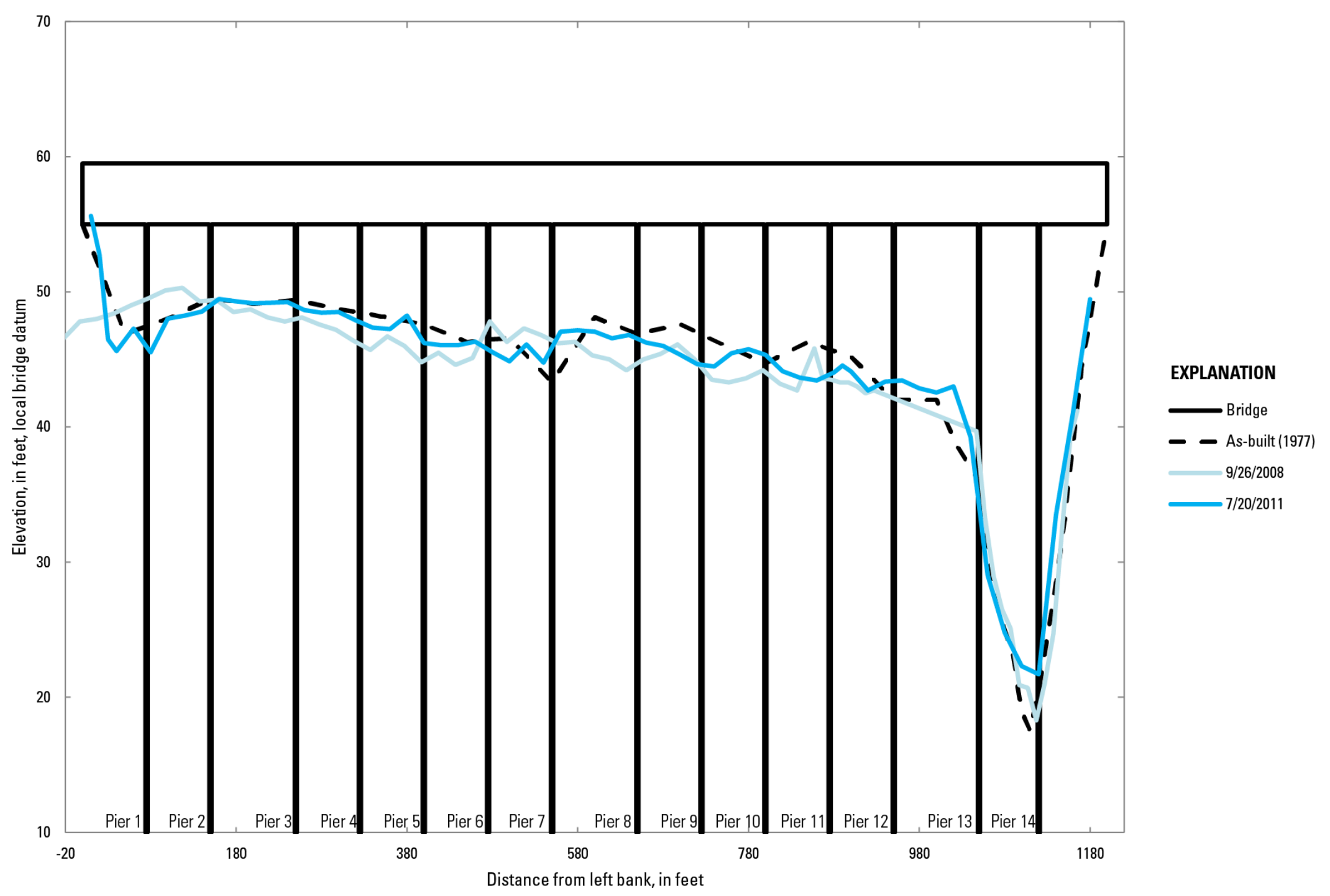

Figure 25. Cross sections showing upstream soundings at bridge 334, Copper River Delta, Alaska, 2008-11. 
BN 336 Copper River Delta, downstream

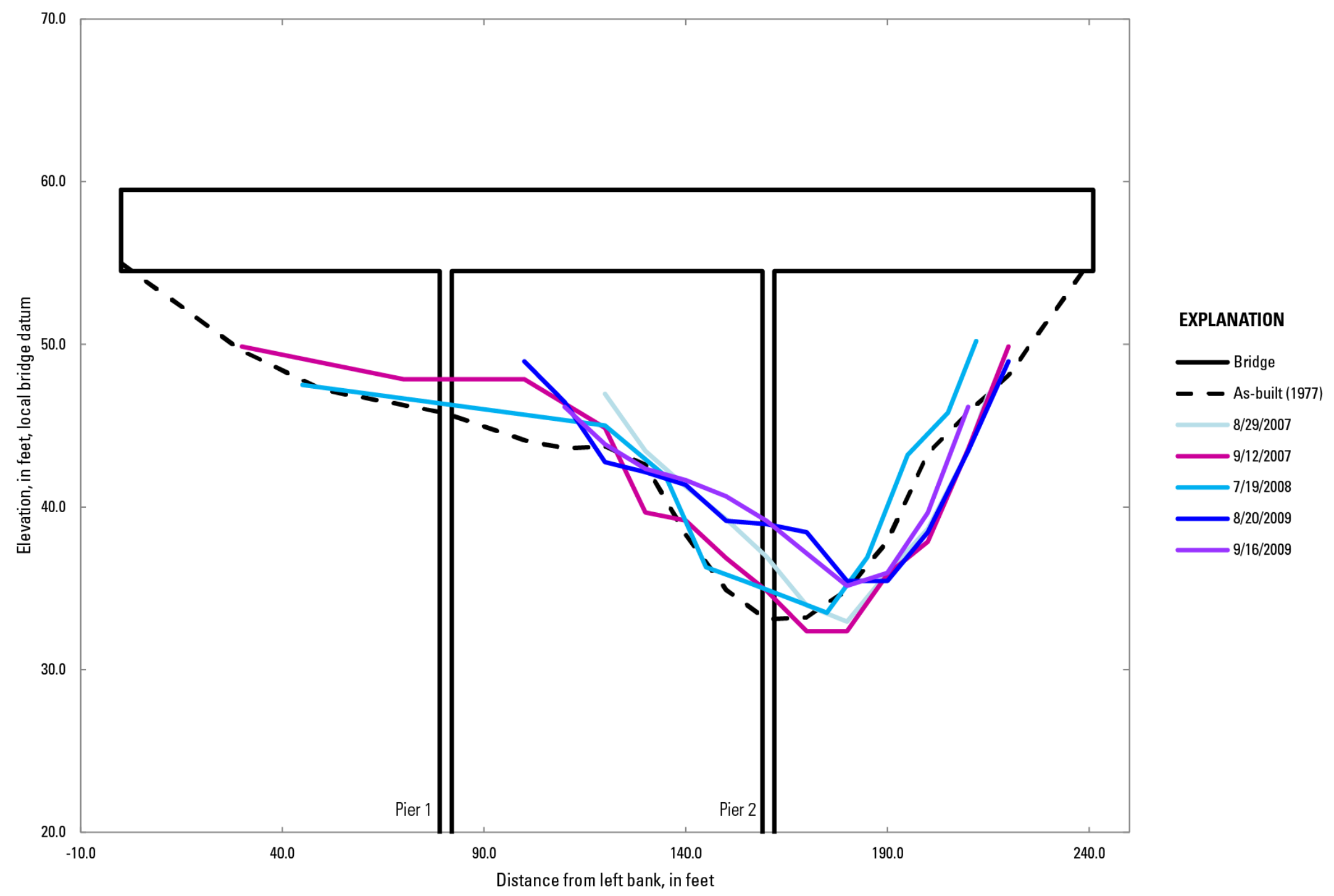

Figure 26. Cross sections showing downstream soundings at bridge 336, Copper River Delta, Alaska, 2007-09. 
BN 339 Copper River Delta, upstream

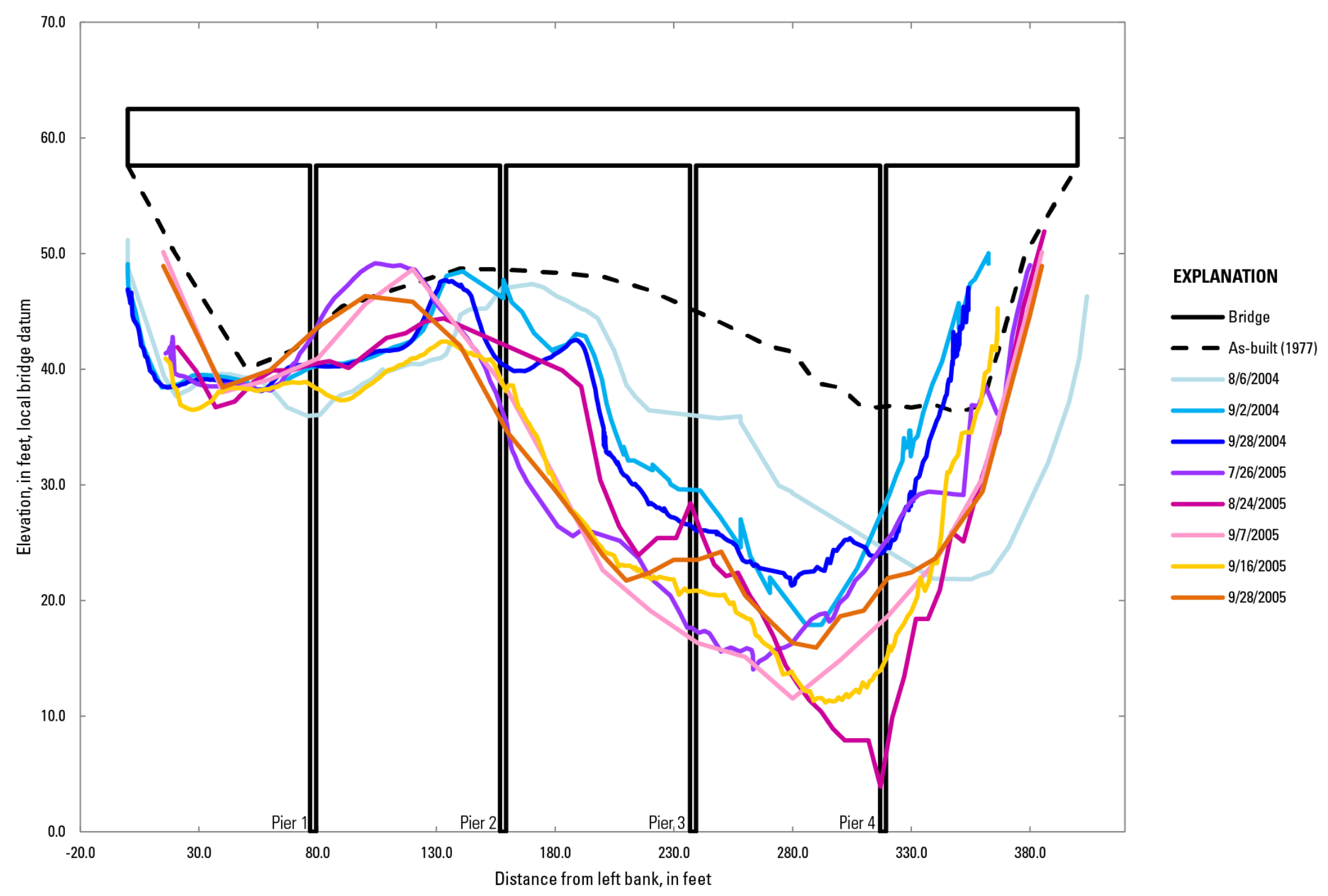

Figure 27. Cross sections showing upstream soundings at bridge 339, Copper River Delta, Alaska, 2004-05. 


\section{BN 339 Copper River Delta, upstream}

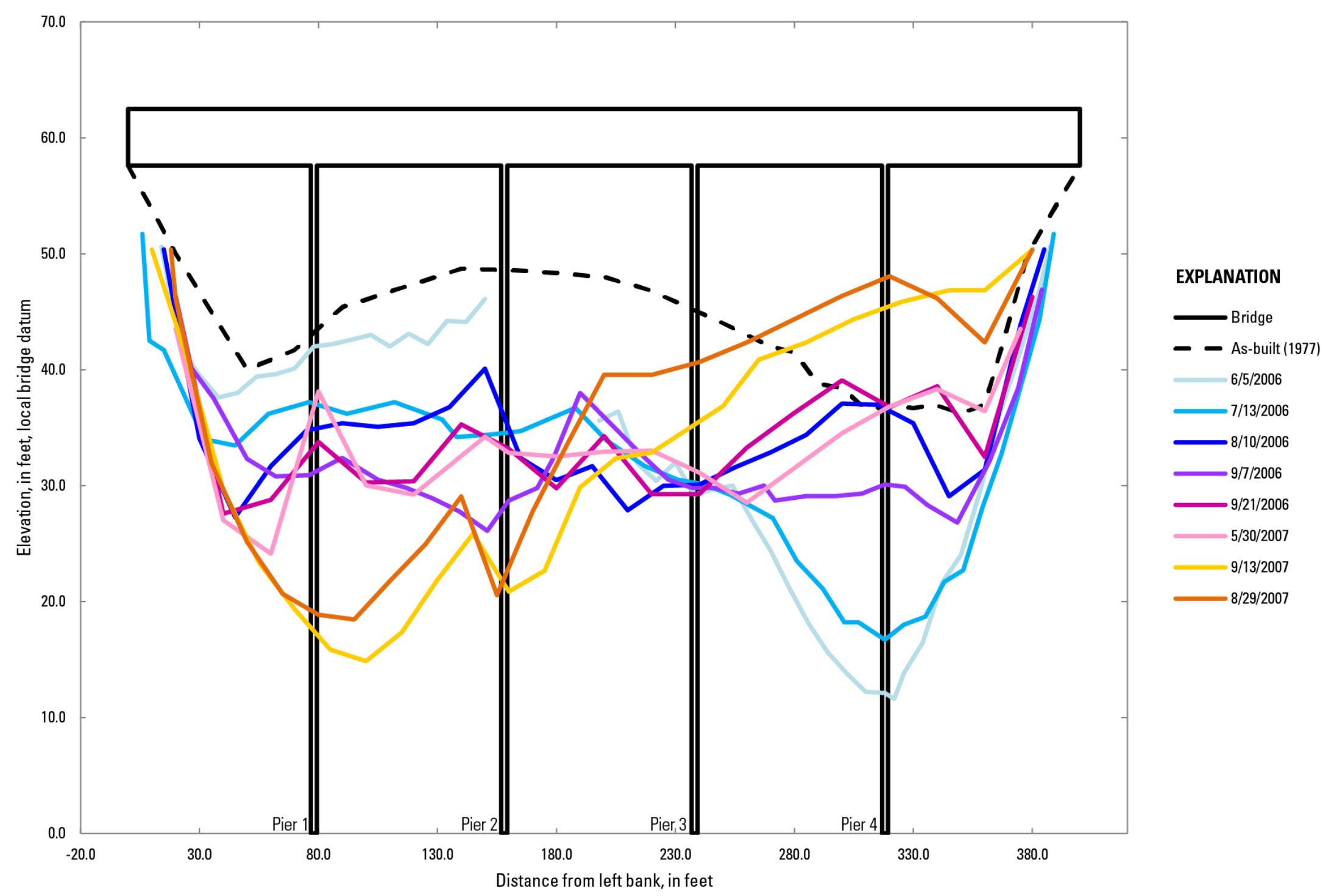

Figure 28. Cross sections showing upstream soundings at bridge 339, Copper River Delta, Alaska, 2006-07. 
BN 339 Copper River Delta, upstream

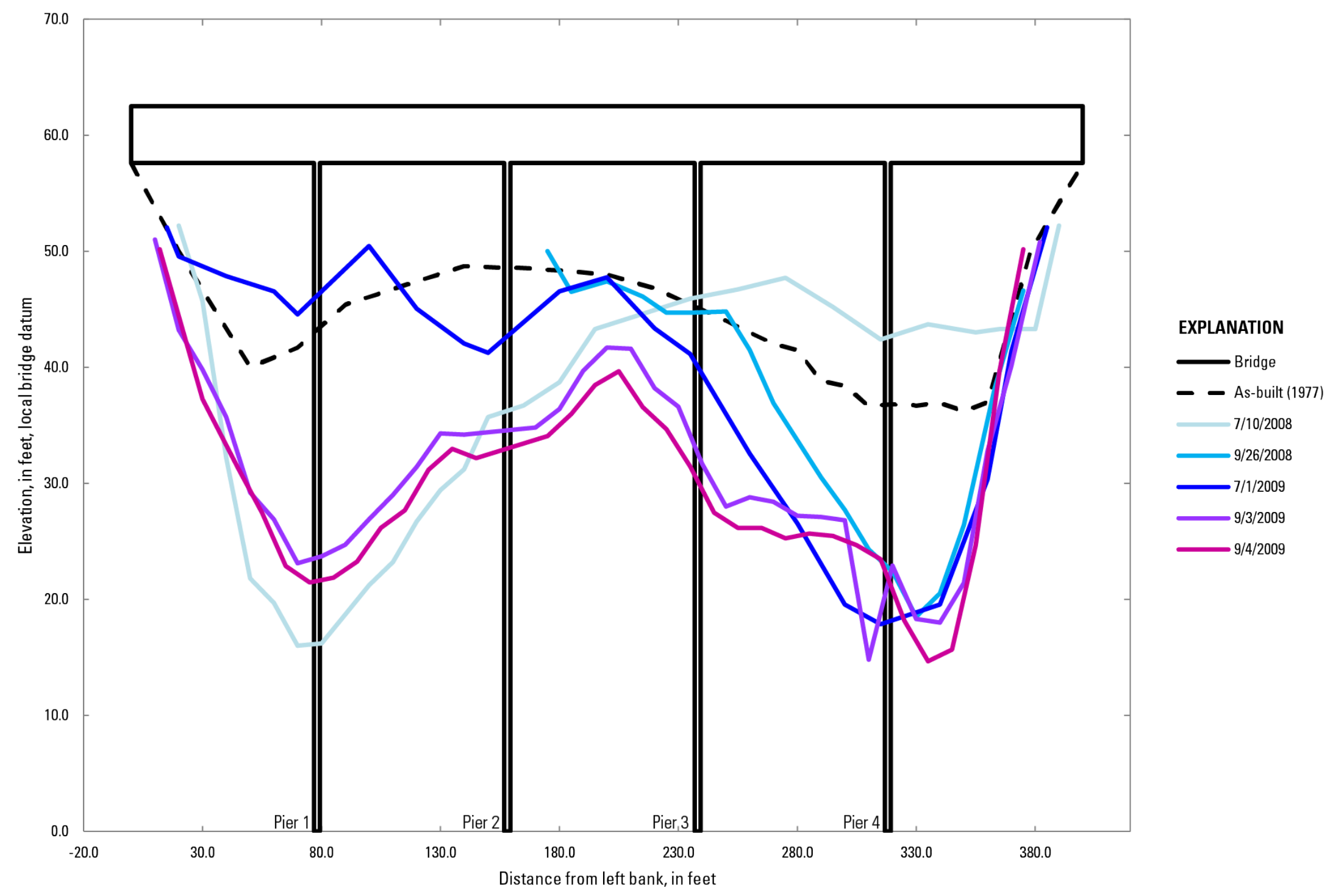

Figure 29. Cross sections showing upstream soundings at bridge 339, Copper River Delta, Alaska, 2008-09. 
BN 339 Copper River Delta, upstream

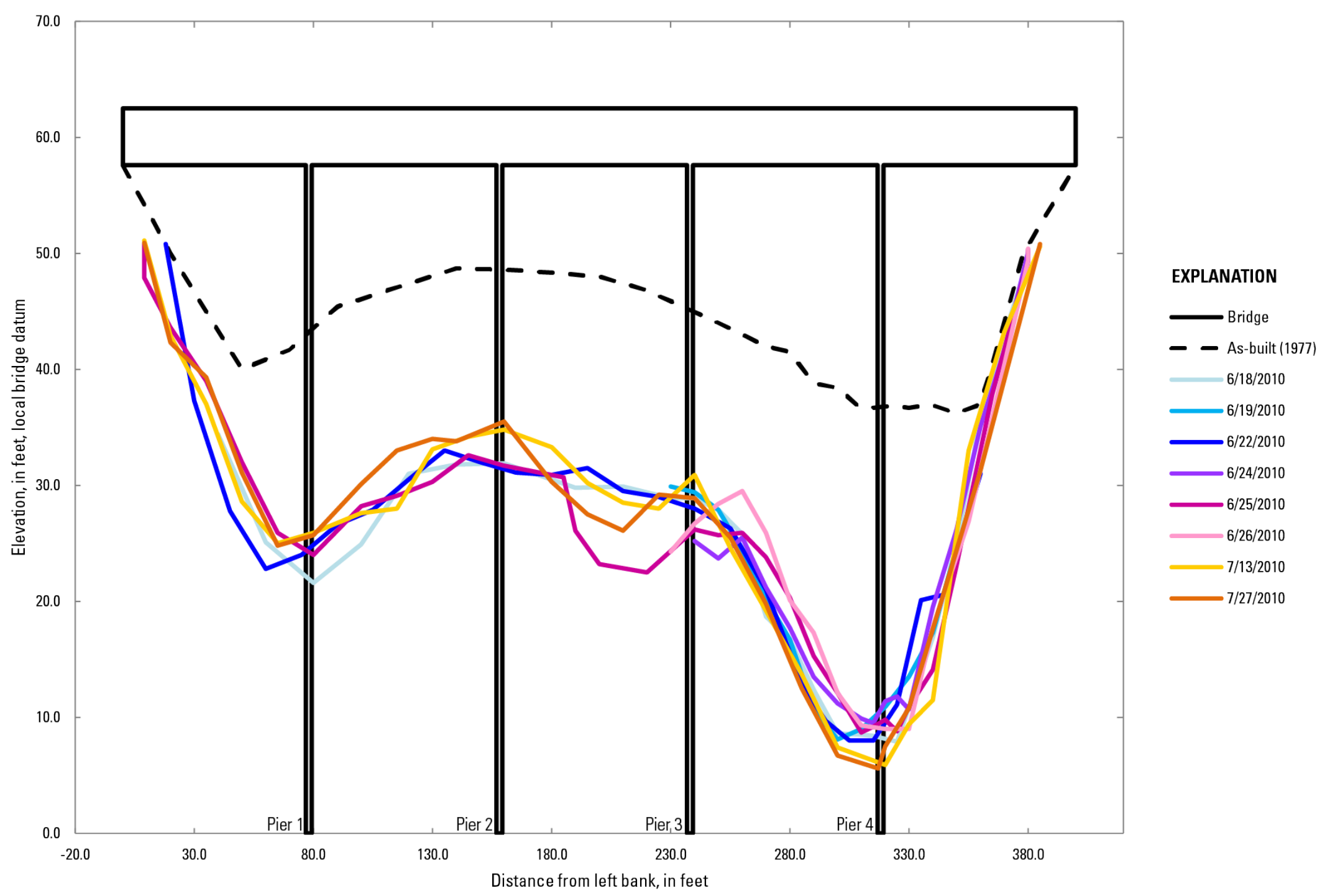

Figure 30. Cross sections showing upstream soundings at bridge 339, Copper River Delta, Alaska, 2010. 
BN 339 Copper River Delta, upstream

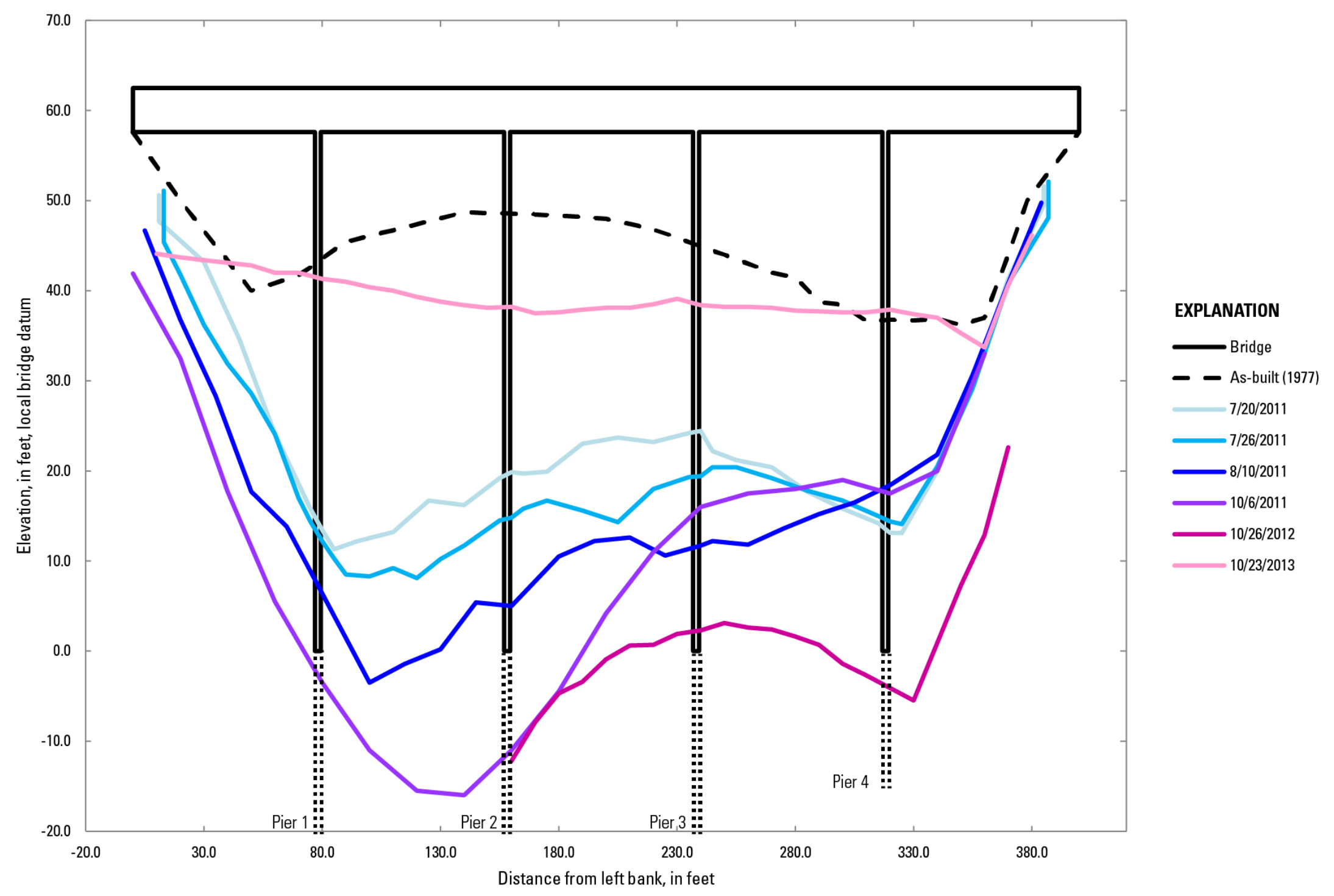

Figure 31. Cross sections showing upstream soundings at bridge 339, Copper River Delta, Alaska, 2011-13. 
BN 339 Copper River Delta, downstream

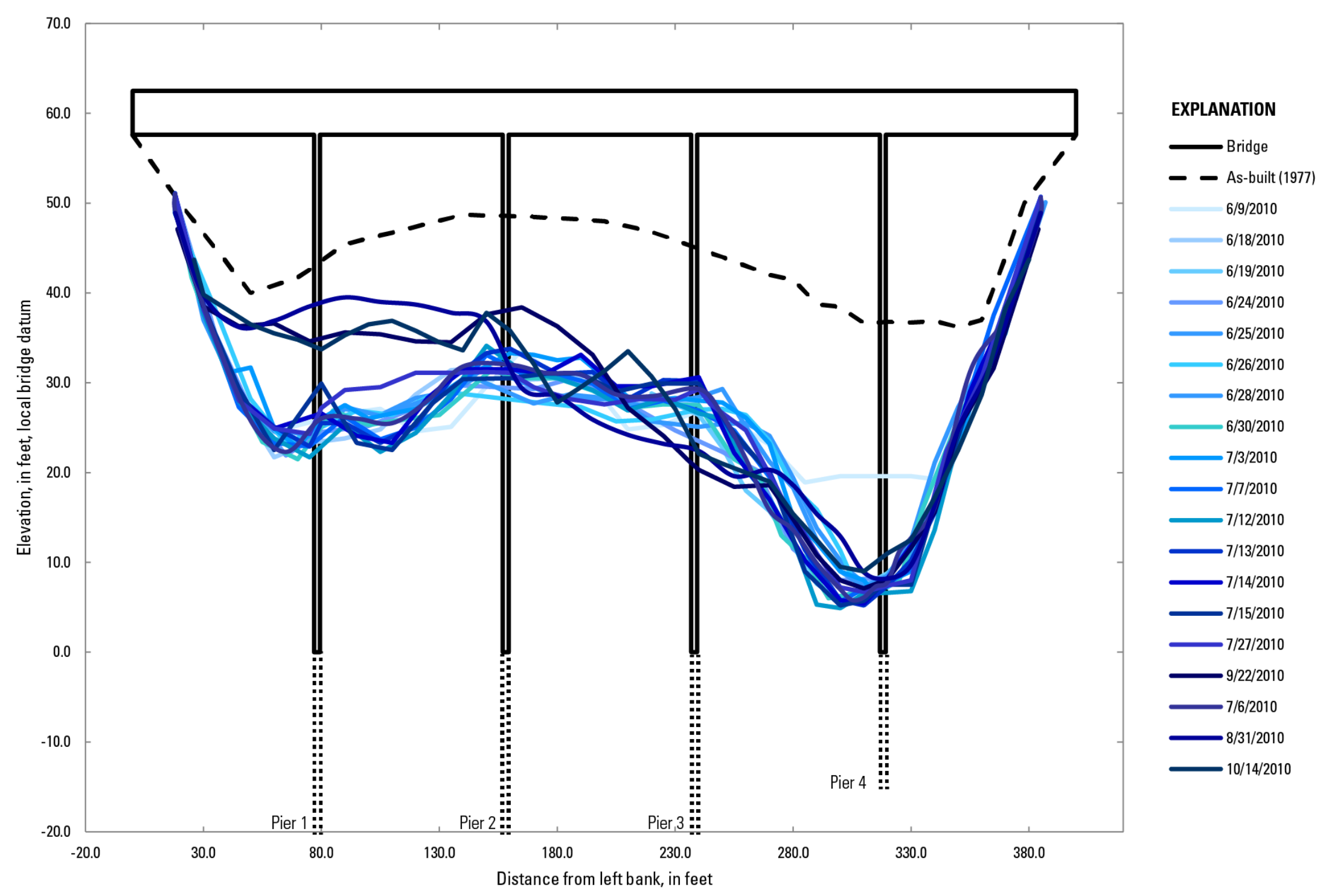

Figure 32. Cross sections showing downstream soundings at bridge 339, Copper River Delta, Alaska, 2010. 
BN 339 Copper River Delta, downstream

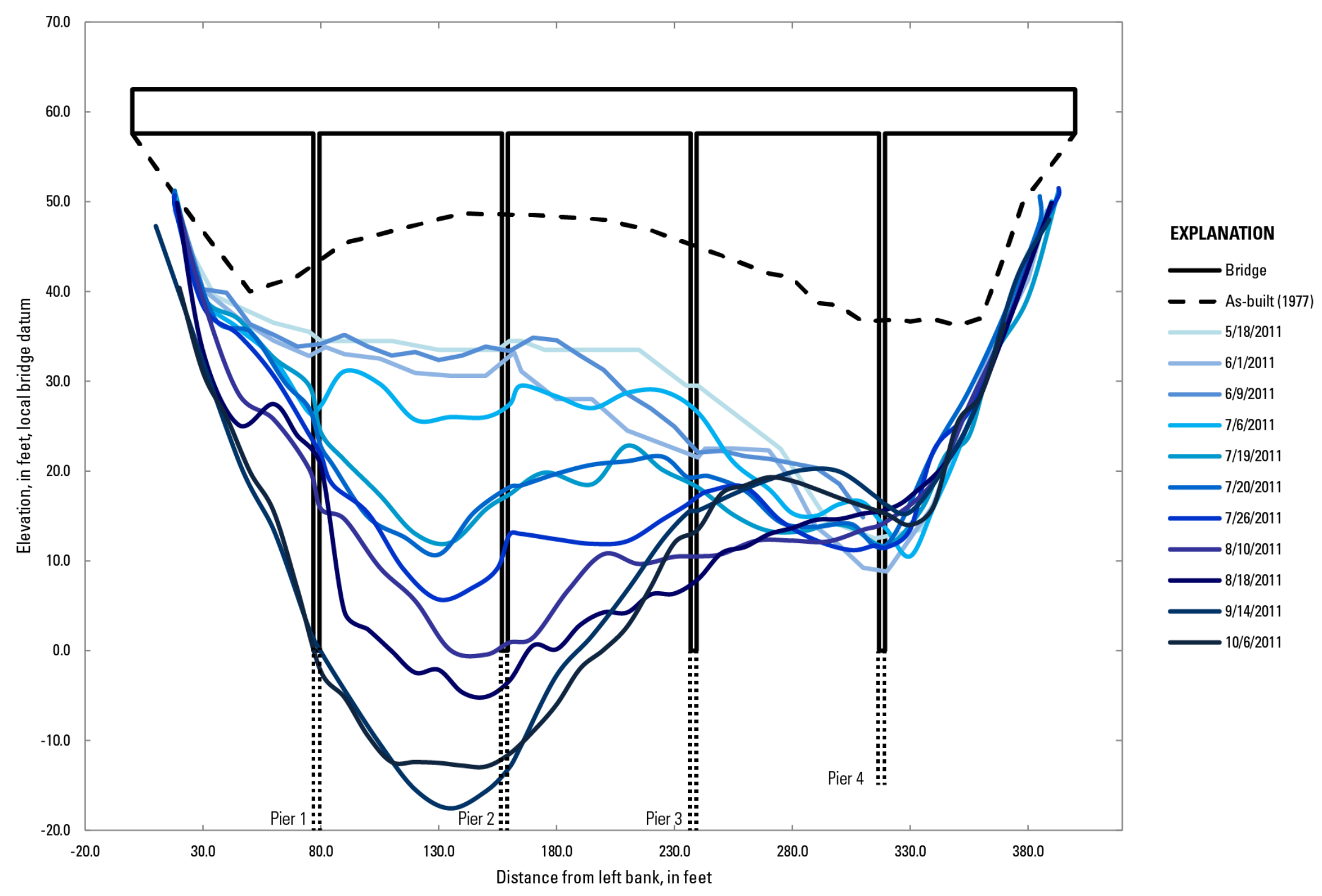

Figure 33. Cross sections showing downstream soundings at bridge 339, Copper River Delta, Alaska, 2011. 
BN 340 Copper River Delta, downstream

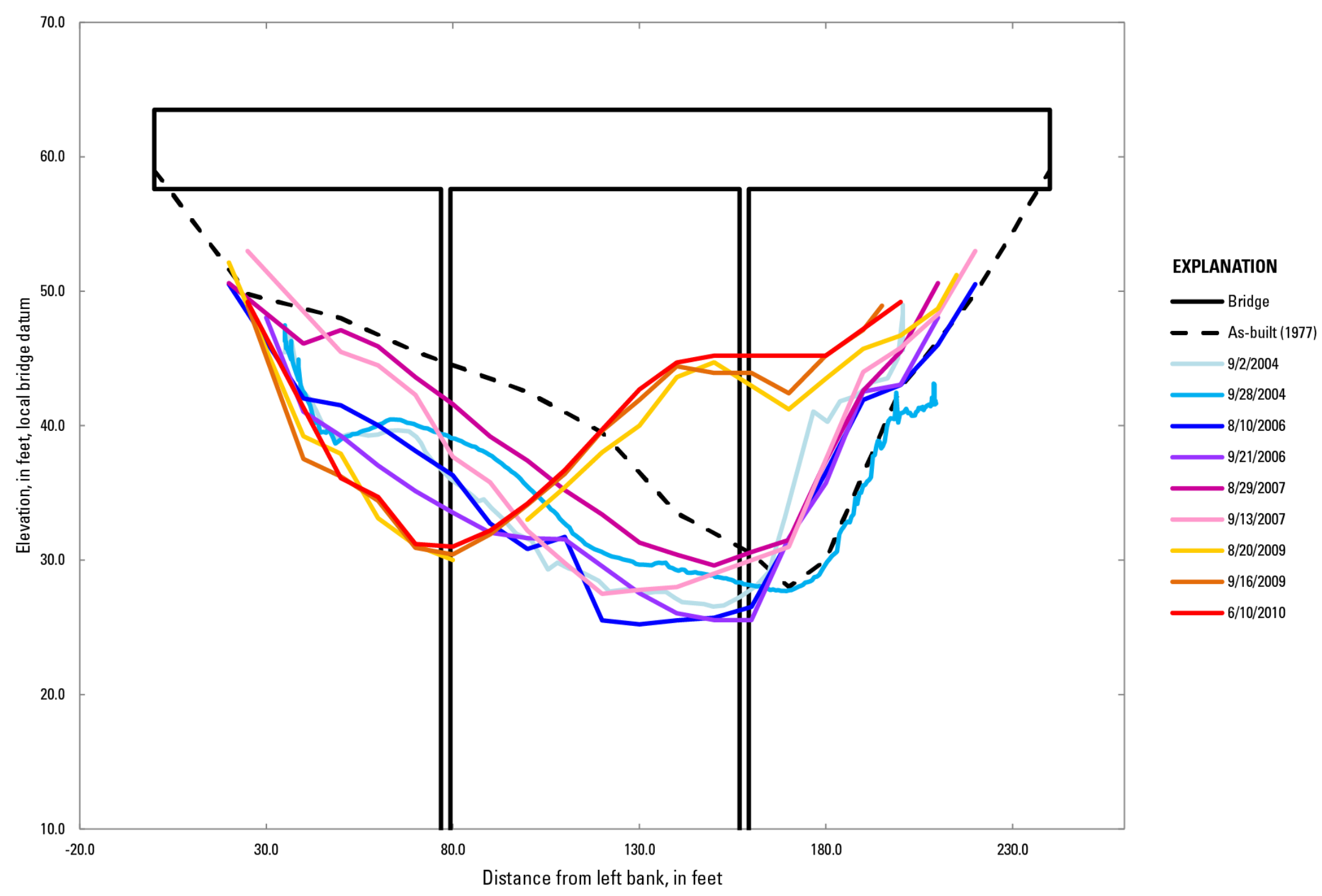

Figure 34. Cross sections showing downstream soundings at bridge 340, Copper River Delta, Alaska, 2004-10. 
BN 340 Copper River Delta, upstream

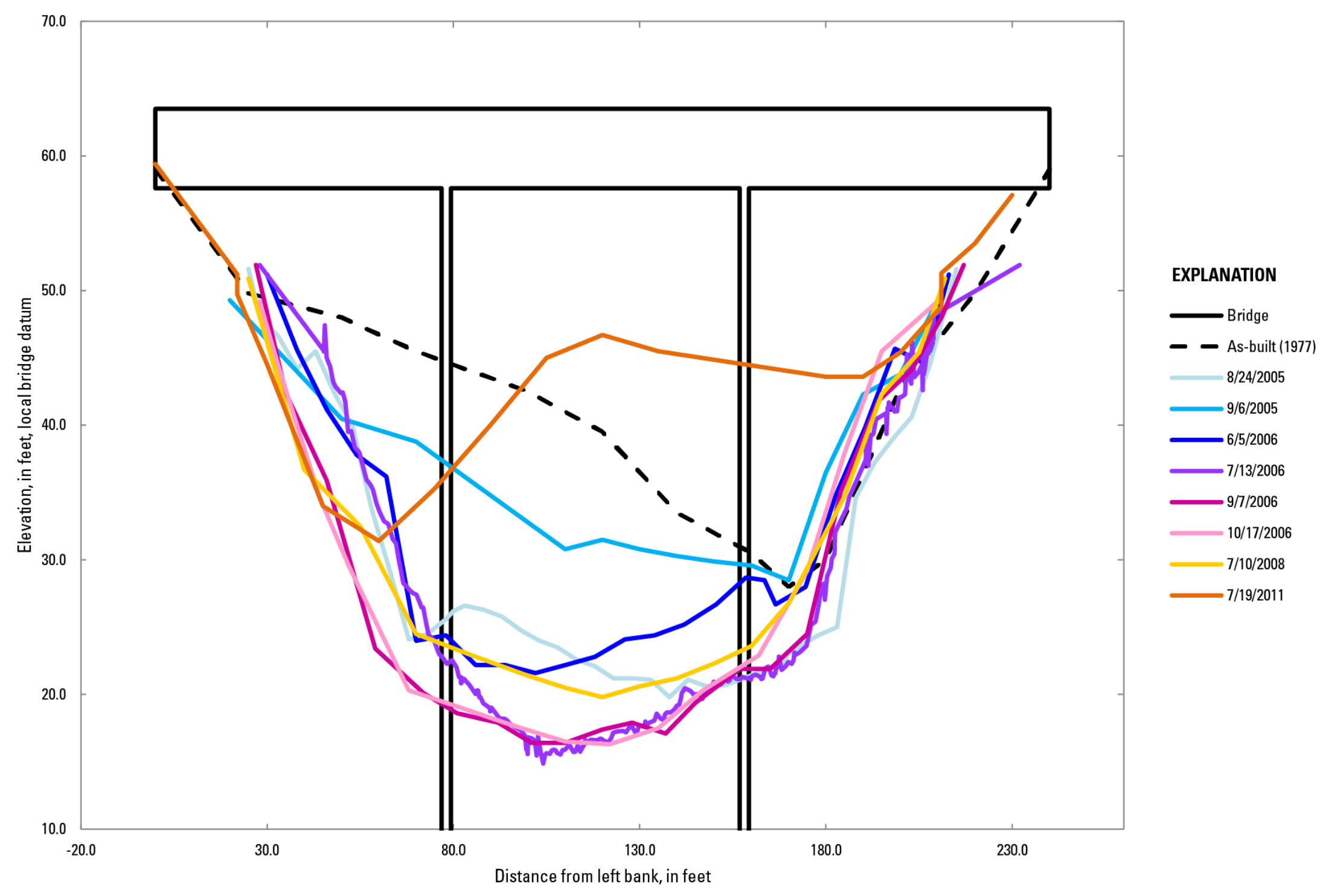

Figure 35. Cross sections showing upstream soundings at bridge 340, Copper River Delta, Alaska, 2005-11. 


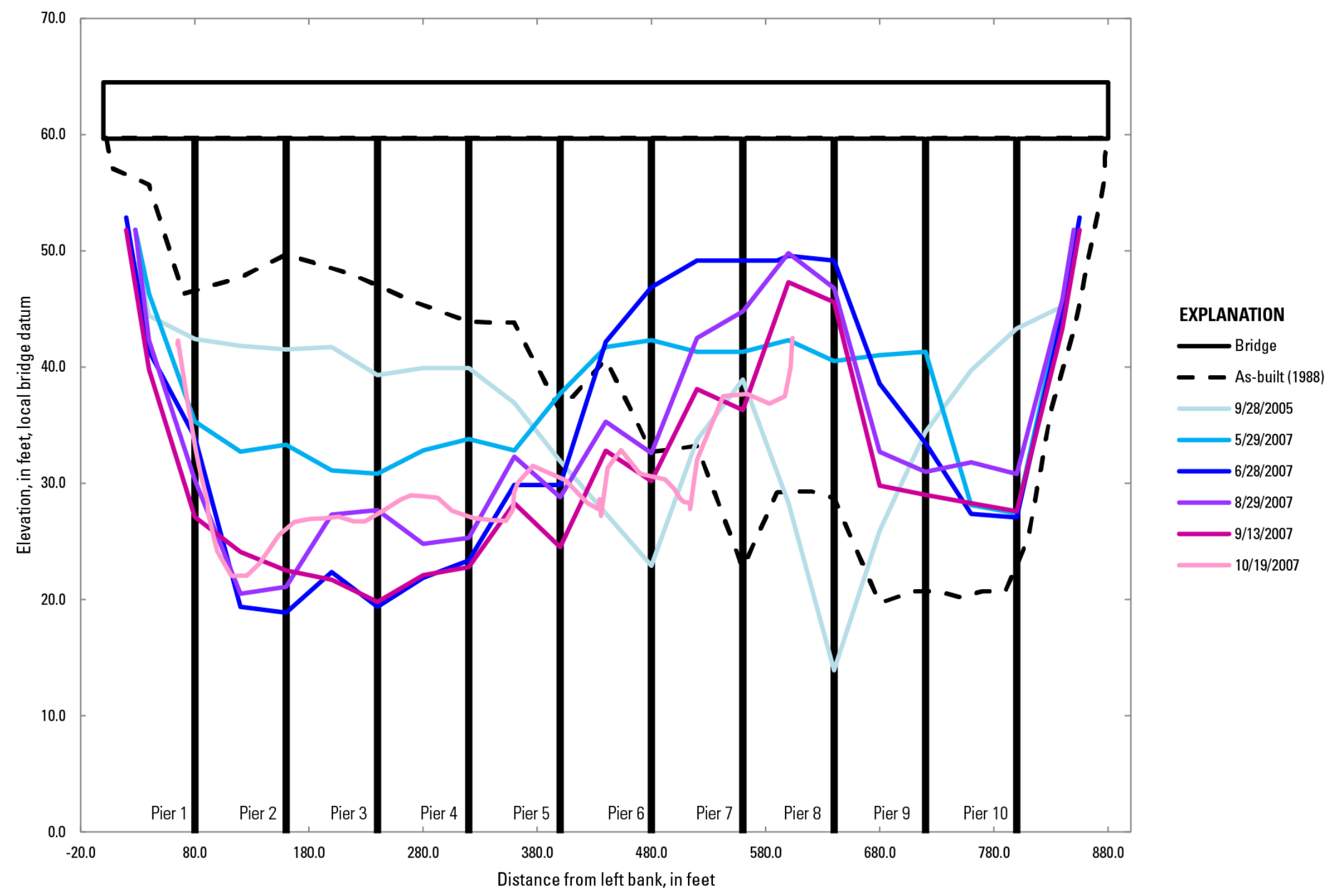

Figure 36. Cross sections showing downstream soundings at bridge 342, Copper River Delta, Alaska, 2005-07. 
BN 342 Copper River Delta, downstream

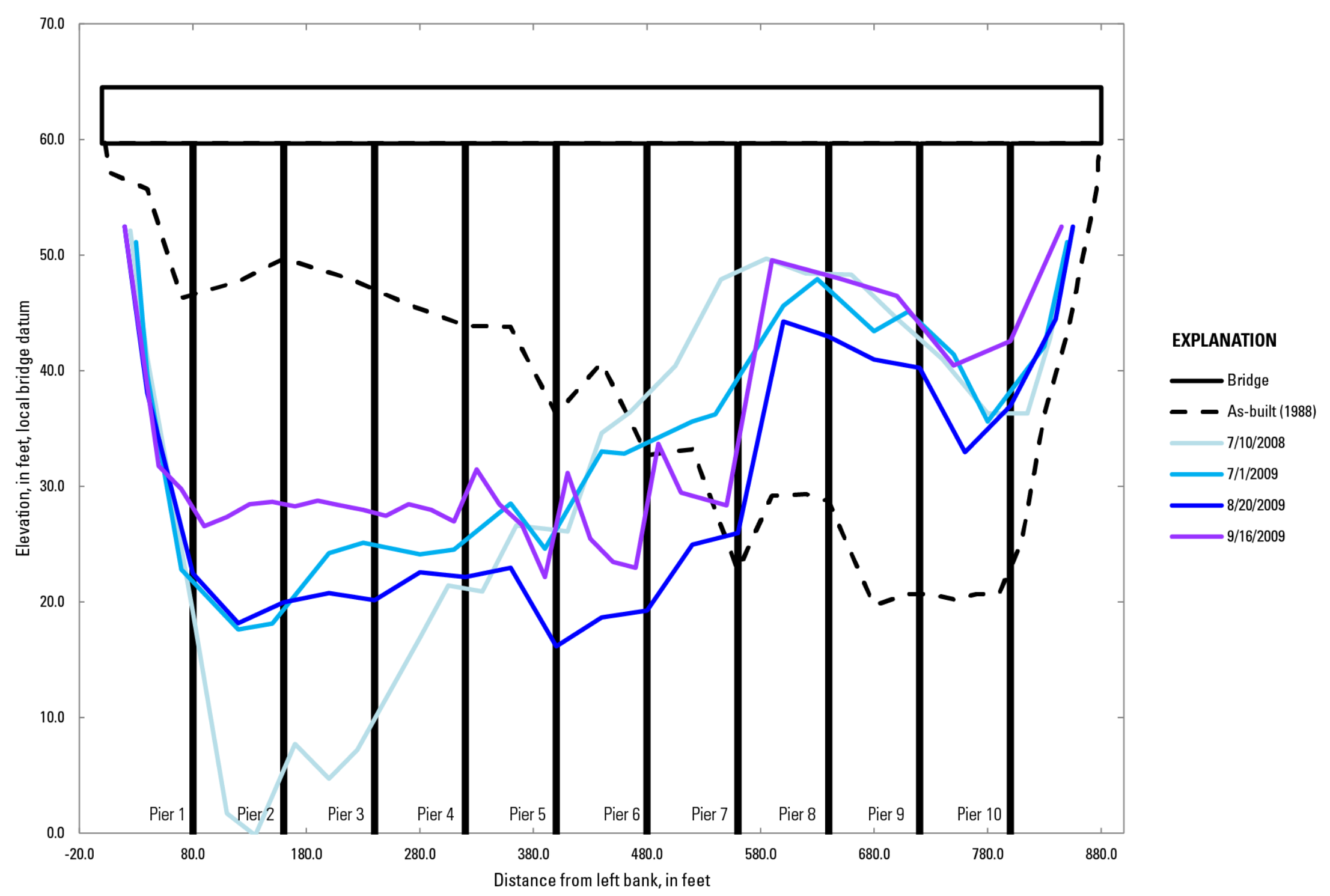

Figure 37. Cross sections showing downstream soundings at bridge 342, Copper River Delta, Alaska, 2008-09. 
BN 342 Copper River Delta, downstream

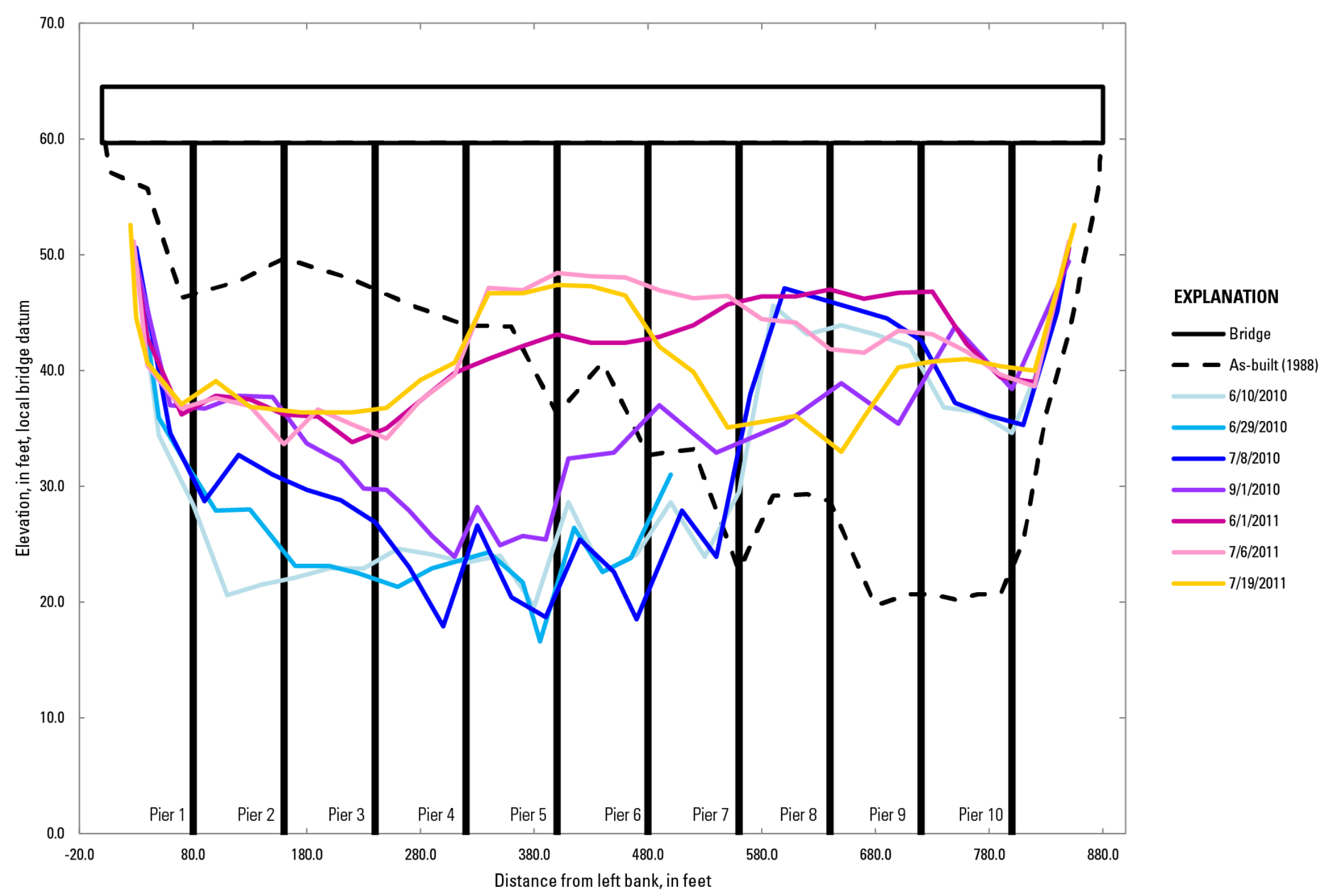

Figure 38. Cross sections showing downstream soundings at bridge 342, Copper River Delta, Alaska, 2010-11. 
BN 342 Copper River Delta, upstream

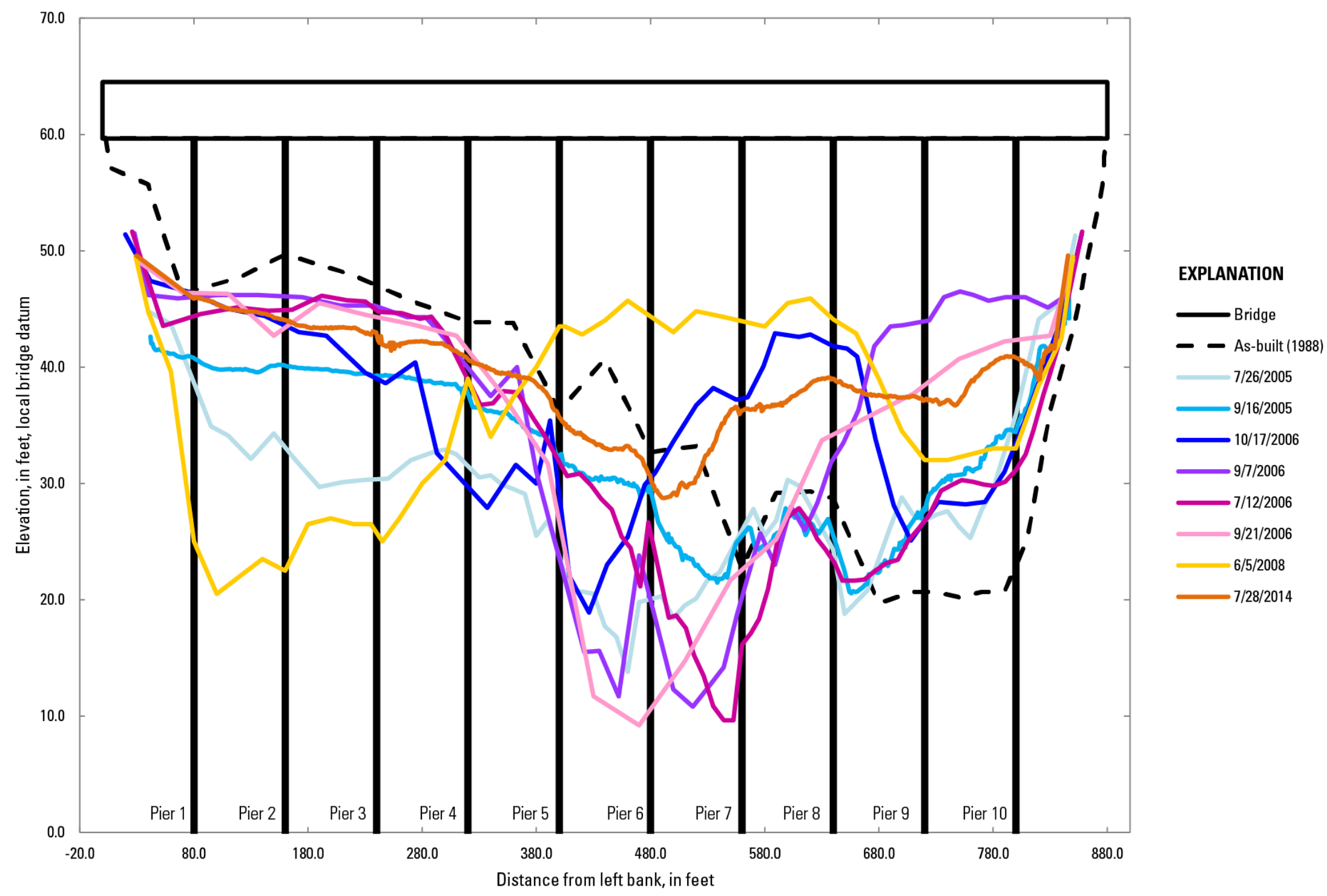

Figure 39. Cross sections showing upstream soundings at bridge 342, Copper River Delta, Alaska, 2005--14. 
BN 1187 Copper River Delta, downstream

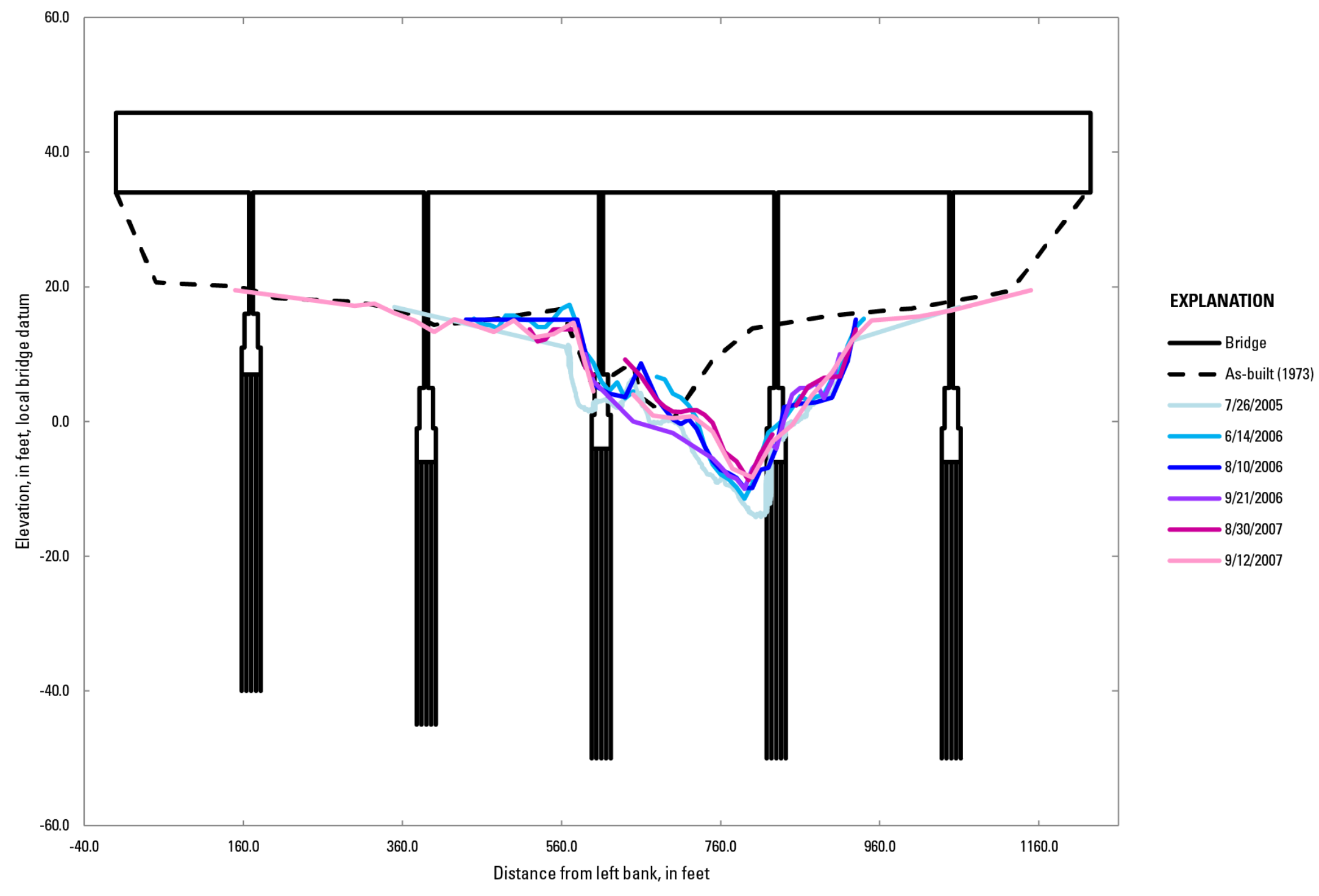

Figure 40. Cross sections showing downstream soundings at bridge 1187, Copper River Delta, Alaska, 2005-07. 
BN 1187 Copper River Delta, downstream

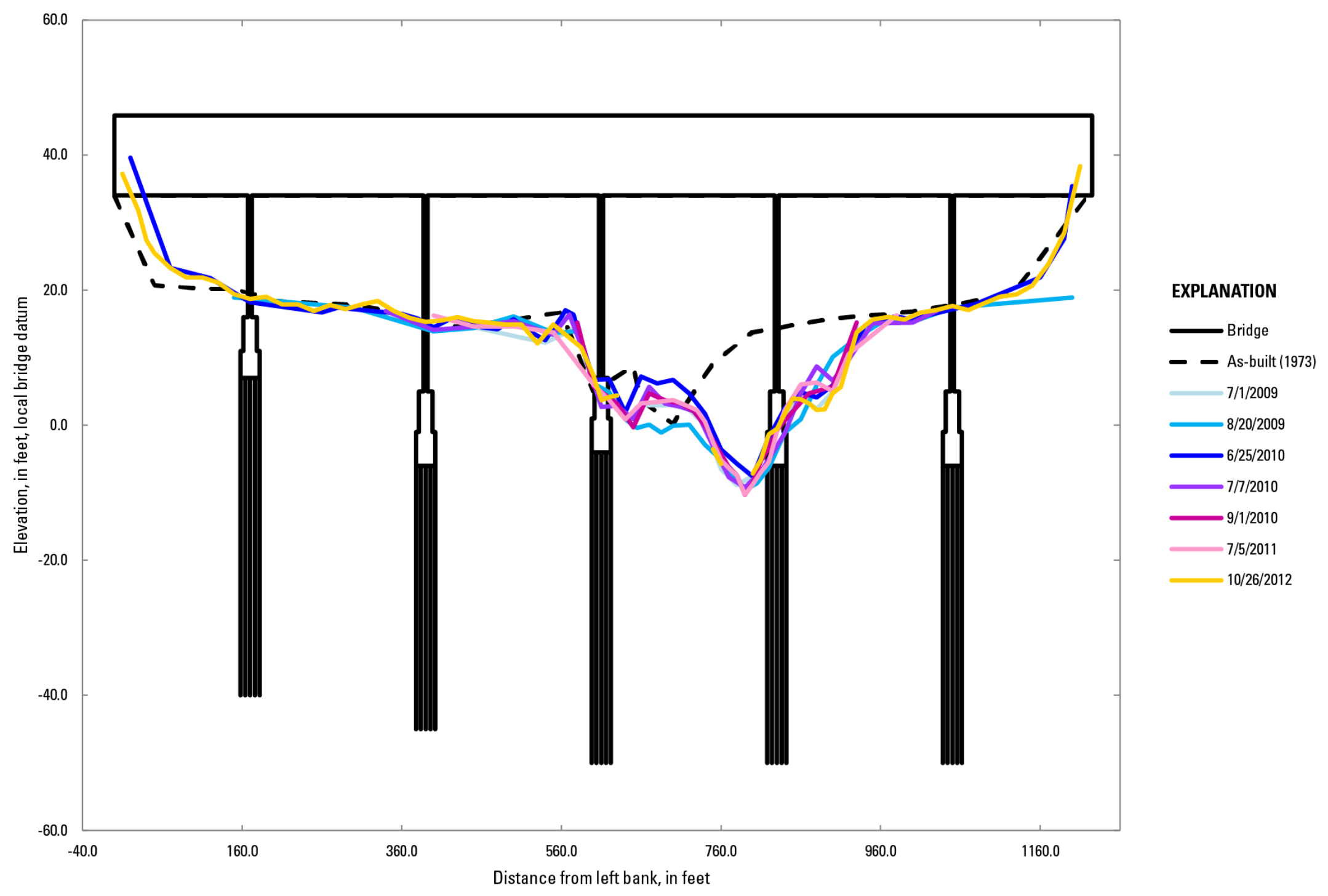

Figure 41. Cross sections showing downstream soundings at bridge 1187, Copper River Delta, Alaska, 2009-12. 


\section{BN 1187 Copper River Delta, downstream}

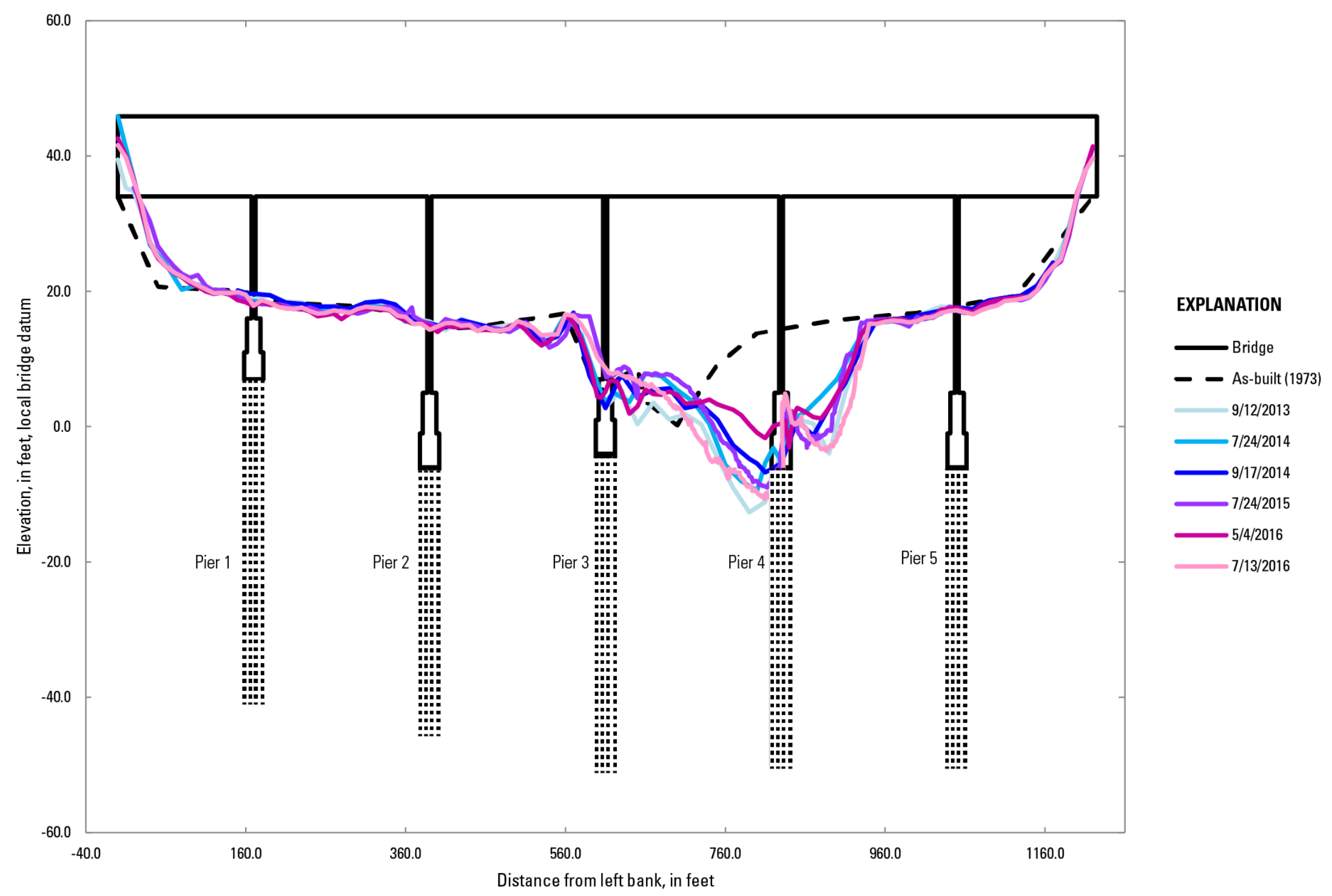

Figure 42. Cross sections showing downstream soundings at bridge 1187, Copper River Delta, Alaska, 2013-16. 
BN 1187 Copper River Delta, upstream

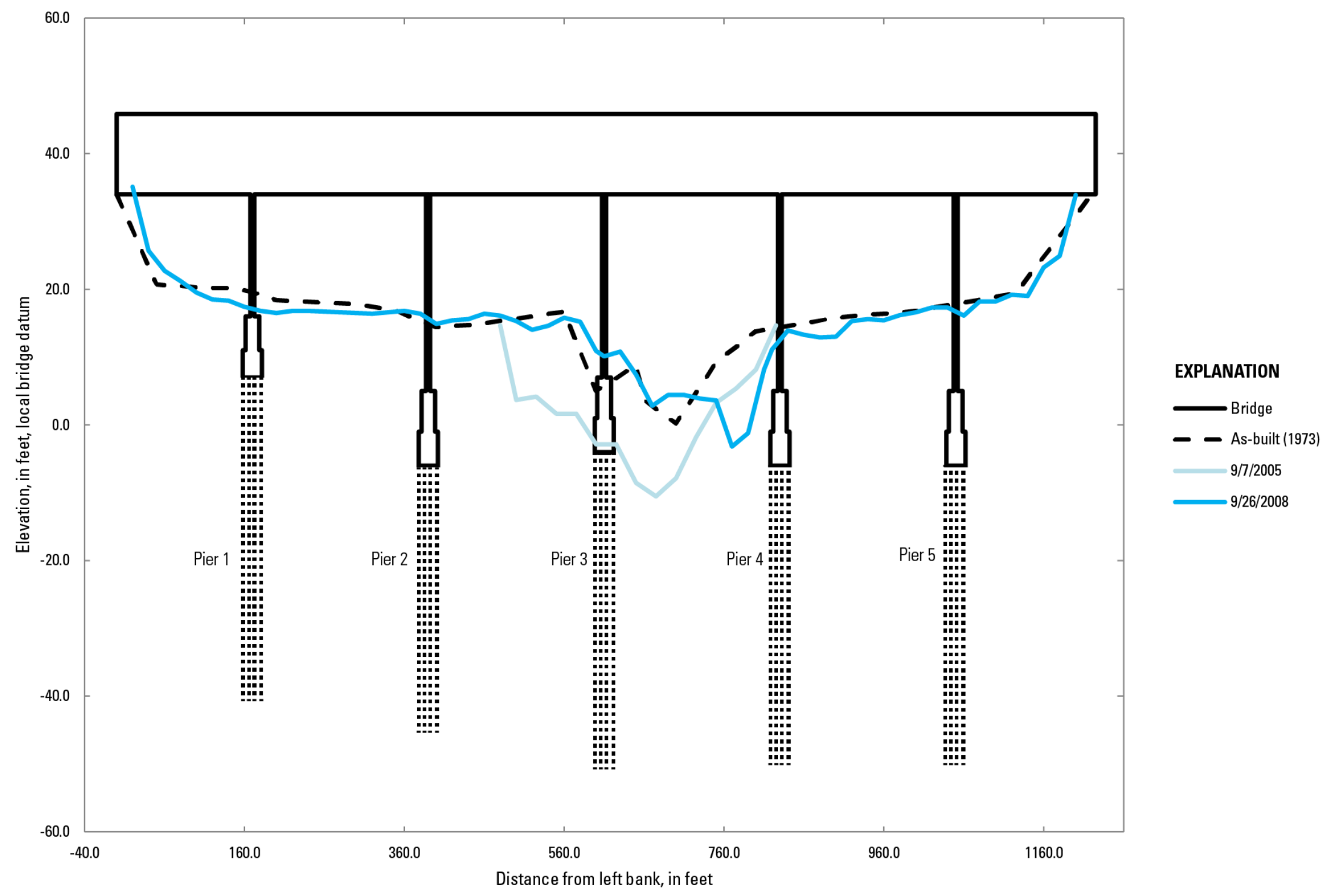

Figure 43. Cross sections showing upstream soundings at bridge 1187, Copper River Delta, Alaska, 2005-08. 


\section{Bridge 505, Tanana River at Tok}

The bridge is located where the Tanana River enters a natural constriction that is controlled by bedrock along the right bank and is a single channel at all but low flows. The river has migrated in the direction of the right bank since the bridge was built and is directed by the bedrock into the bridge reach at an angle. This flow angle of attack on the pier was observed to be about 40-45 degrees (Conaway and Moran, 2004). The high angle of attack on the pier and position of the bridge at the head of a natural constriction, where flow velocities and sediment transport capacity are the highest, made this structure particularly susceptible to streambed scour (Conaway and Moran, 2004). An extensive bathymetric survey was done at Bridge 505 in 2003 (Conaway and Moran, 2004) to build a two-dimensional hydrodynamic model. Annual cross-sectional surveys were done from 2002 to 2010 (figs. 44 and 45). Scour of as much as $22 \mathrm{ft}$ was concentrated at the right-bank pier. Some lesser contraction scour of $9 \mathrm{ft}$ also was noticed at this crossing. The bridge was ultimately replaced in 2010, $250 \mathrm{ft}$ downstream of the original bridge (Conaway, 2010). 
BN 505 Tanana River at Tok, upstream

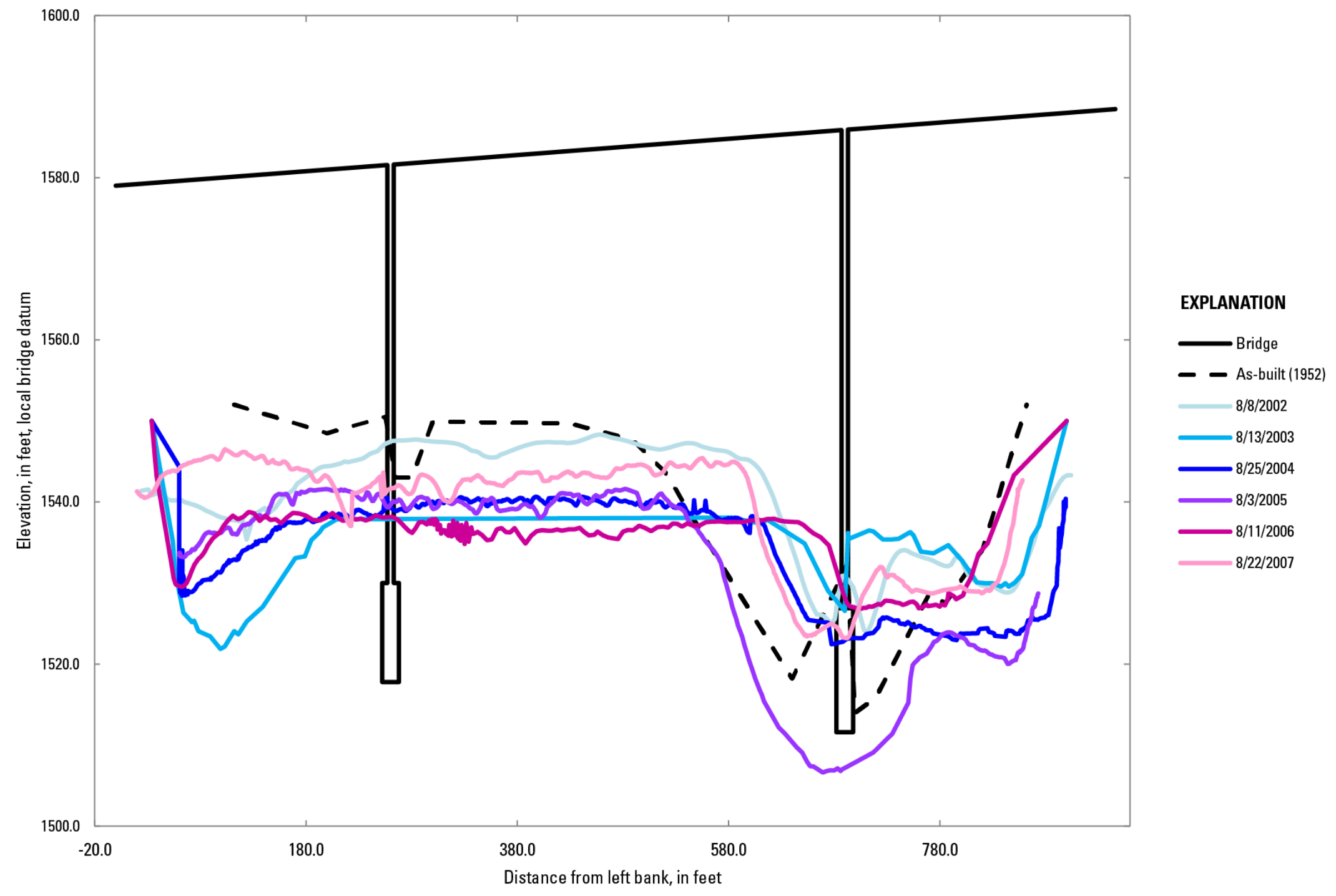

Figure 44. Cross sections showing upstream soundings at bridge 505, Tanana River at Tok, Alaska, 2002-07. 
BN 505 Tanana River at Tok, upstream

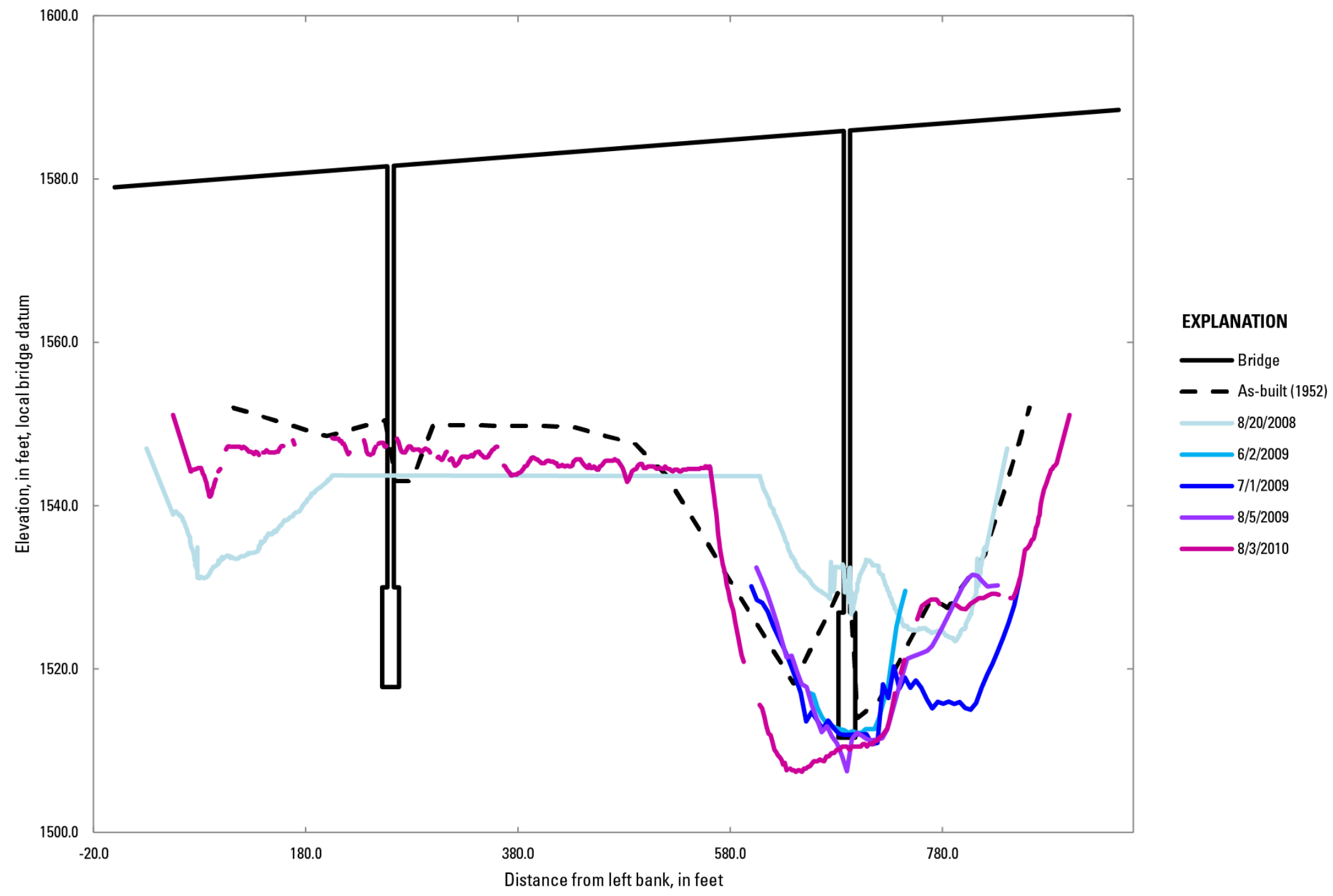

Figure 45. Cross sections showing upstream soundings at bridge 505, Tanana River at Tok, Alaska, 2008-10. 


\section{Bridge 524, Tanana River at Big Delta*}

The Tanana River is in a single channel at the bridge 524 crossing and is joined by the Delta River immediately downstream of the bridge. The USGS initially identified the potential for streambed scour at the bridge in 1975 (Norman, 1975). A detailed hydraulic survey and scour investigation in 1996 quantified the effects of the downstream confluence of the Delta River on scour at bridge 524 and described the processes responsible for channel erosion (Heinrichs and others, 2006). The angle of approaching flow on the piers is partially controlled by the Delta River and a maximum value of 35 degrees has been observed (Norman, 1975). Cross-sectional surveys were done in 1971 and during 2004-10 and 2012-14 (figs. 46 and 47) and indicate that contraction scour and pier scour occur at this bridge. Erosion along the right bank has been attributed to the complex hydraulics associated with a slough that enters the Tanana River immediately upstream of the bridge and backwater from the Delta River entering immediately downstream of the bridge (Heinrichs and others, 2006). Local scour at the piers is evident in several of the cross sections during years 1971, 2007 and 2010. Pier footings were exposed during 2004 and 2007 soundings. Scour was deepest on the downstream edge of the bridge piers, a finding that is consistent with Norman (1975) who also reported that the minimum streambed elevation at all piers occurred slightly downstream at Big Delta. Channel degradation in more recent years has been confined to the right section of the channel. Between the last pier and the right bank, a maximum of $16 \mathrm{ft}$ of scour was observed. The left section of the channel has some aggradation and degradation over the years but is relatively the same the as-built plan. Maximum localized scour of $11 \mathrm{ft}$ occurred around the piers. 
BN 524 Tanana River at Big Delta, upstream

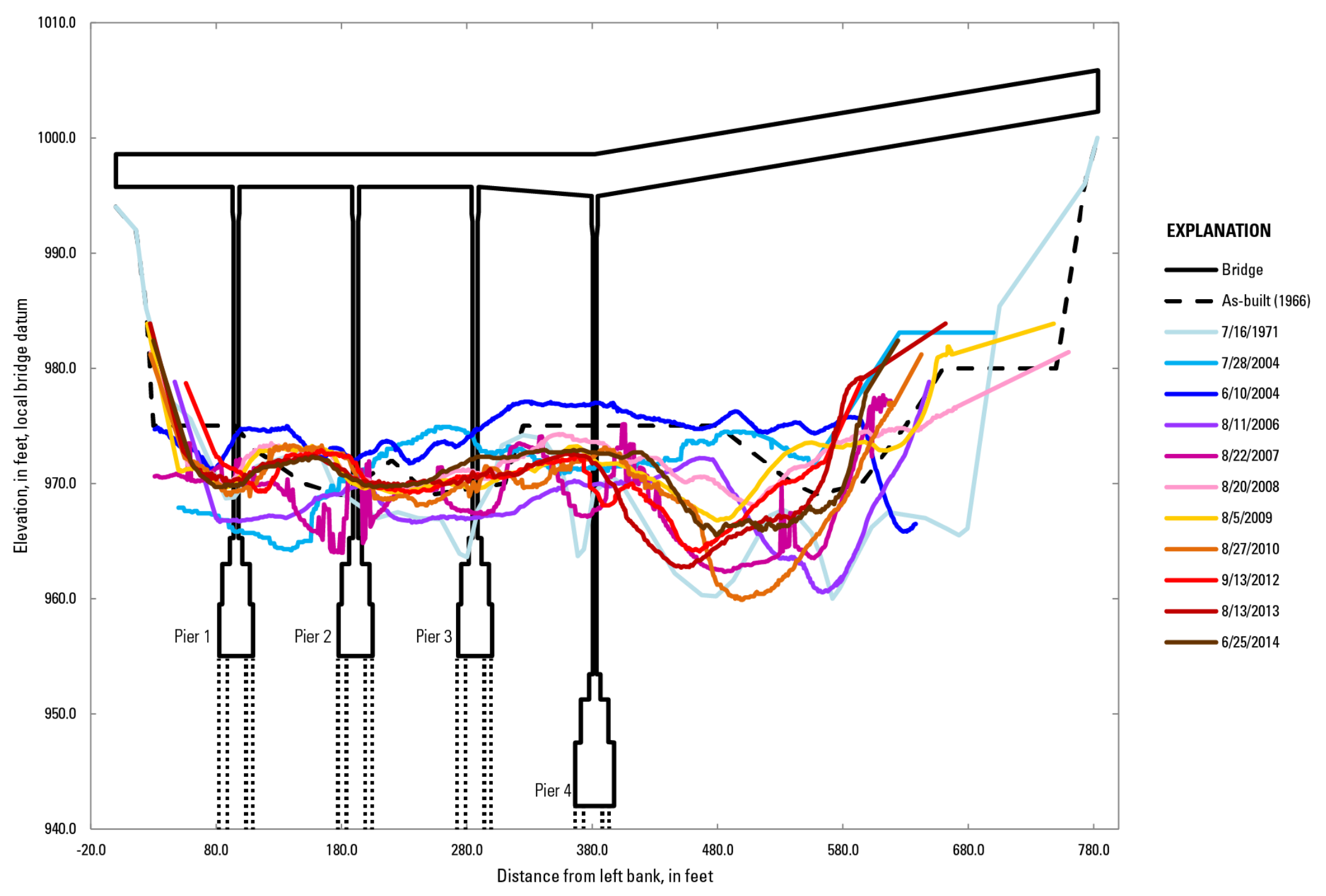

Figure 46. Cross sections showing upstream soundings at bridge 524, Tanana River at Big Delta, Alaska, 1971-2014. 
BN 524 Tanana River at Big Delta, downstream

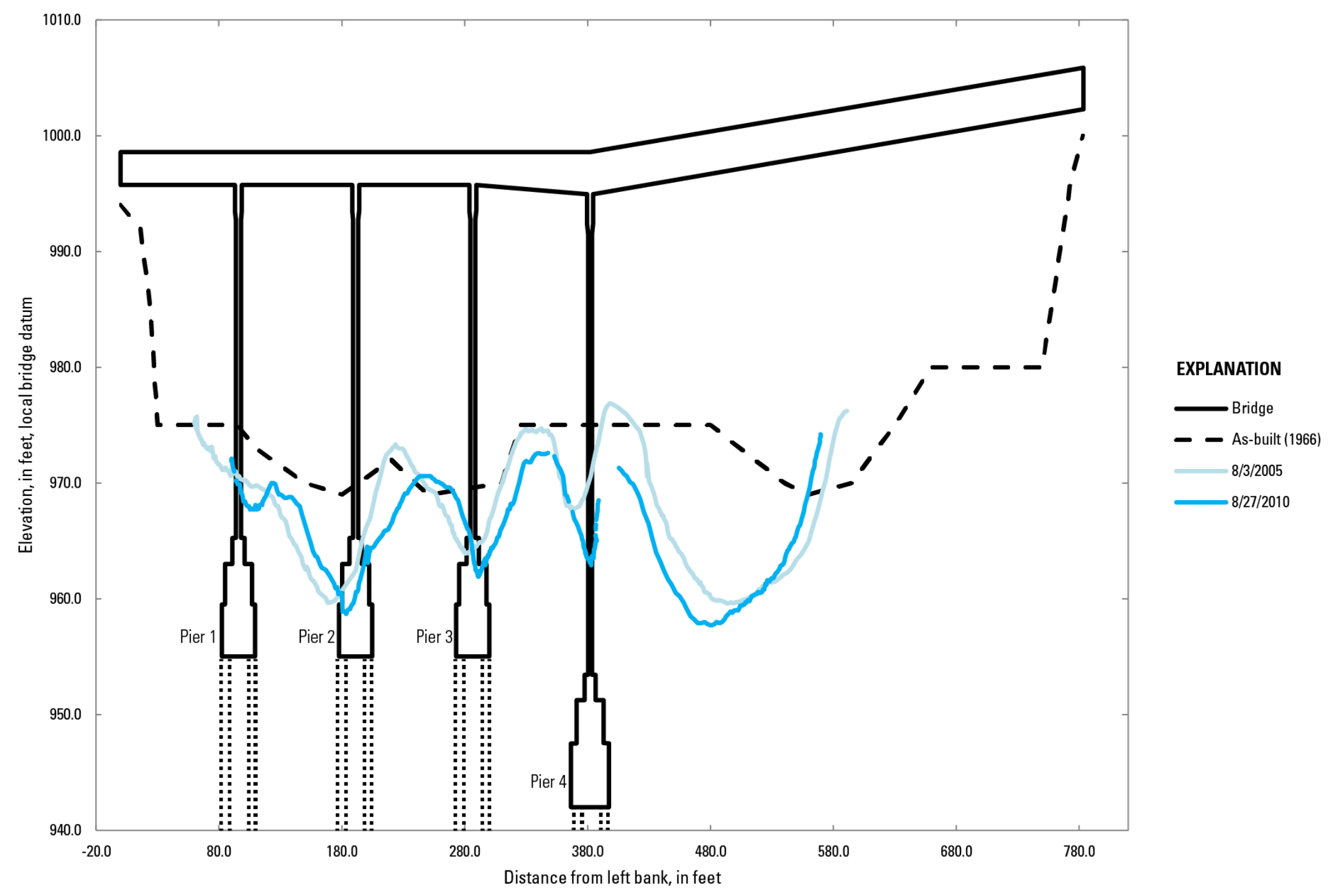

Figure 47. Cross sections showing downstream soundings at bridge 524, Tanana River at Big Delta, Alaska, 2005-10. 


\section{Bridge 527, Salcha River*}

The Salcha River is a major tributary of the Tanana River. It is a single strand through the bridge reach, with slight bends both upstream and downstream of the bridge. The main flow typically is confined to the left side of the channel and the right side of the channel is composed of brushy lowlands that are exposed during low-to-moderate flow but flooded during high flow. There is no substantial angle of attack on the piers; however, the piers tend to collect large amounts of debris during high flows that contribute to the localized scour around the piers (Norman, 1975). Cross-sectional surveys were done during 1995 and 2001-16 (figs. 48-52). The soundings across the channel generally indicate very little long-term aggradation or degradation. Maximum localized scour around the piers of as much as 8 $\mathrm{ft}$ has occurred, and a pier footing was exposed during a channel survey in 2002. 
BN 527 Salcha River, upstream

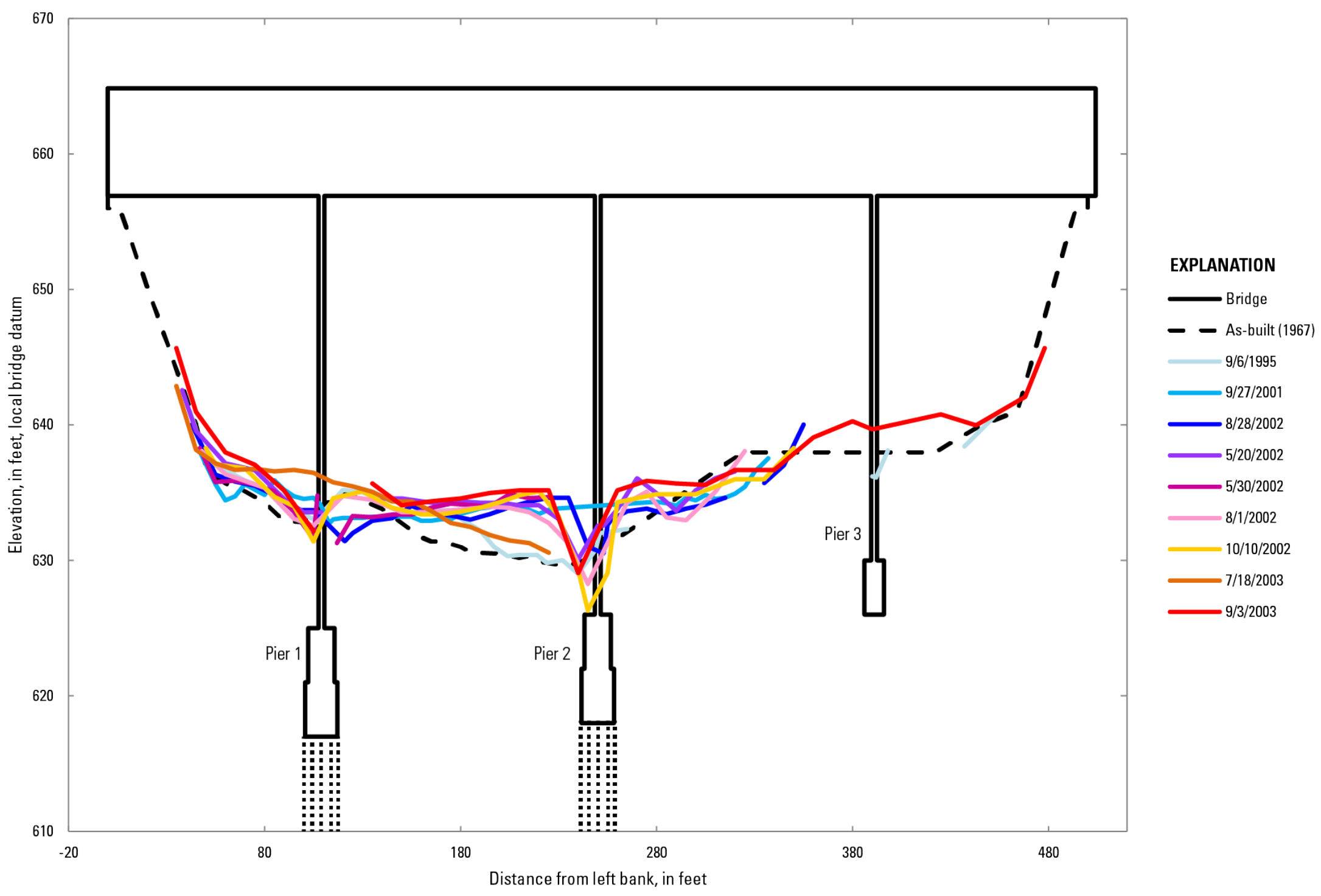

Figure 48. Cross sections showing upstream soundings at bridge 527, Salcha River, Alaska, 1995-2003. 
BN 527 Salcha River, upstream

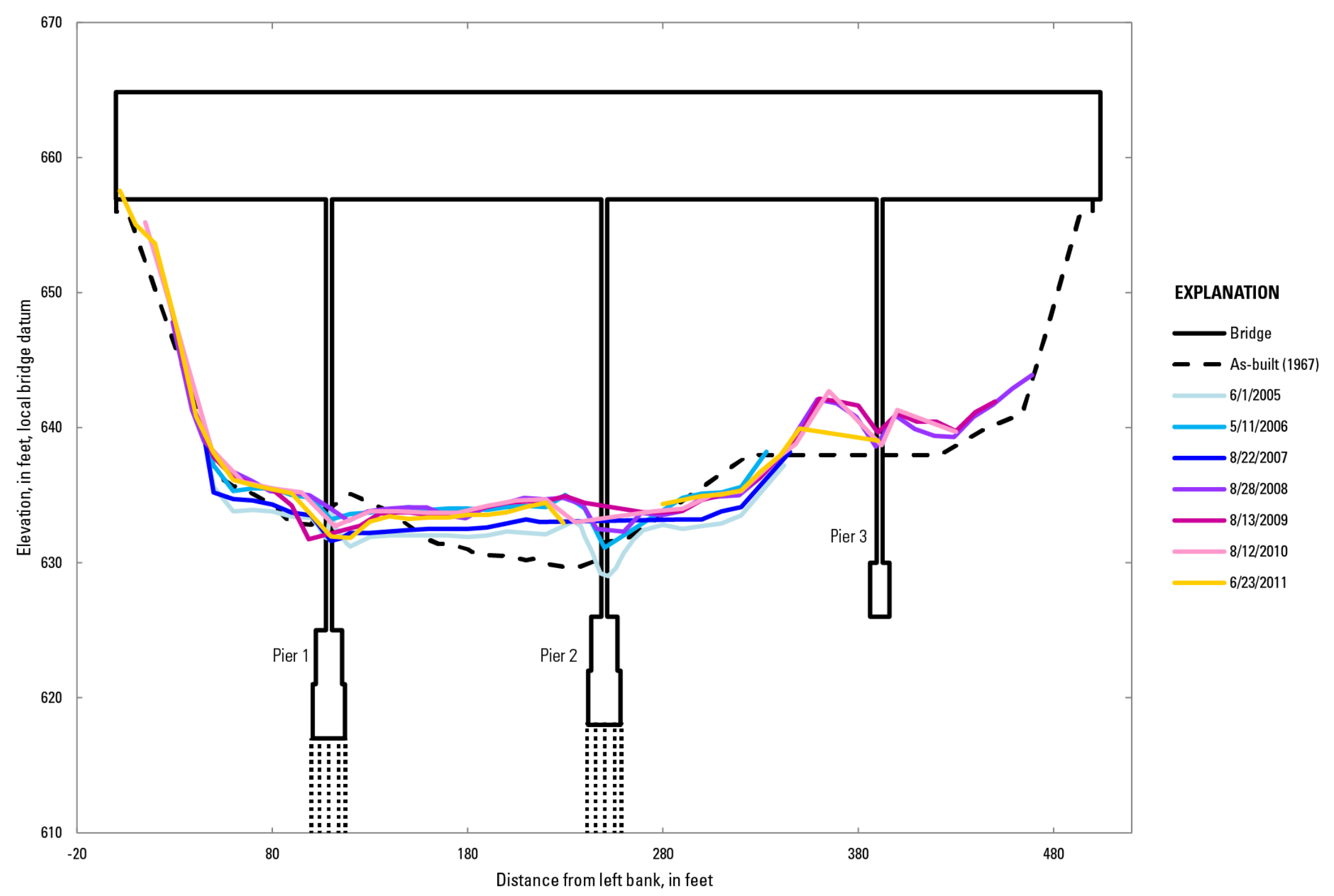

Figure 49. Cross sections showing upstream soundings at bridge 527, Salcha River, Alaska, 2005-11. 
BN 527 Salcha River, upstream

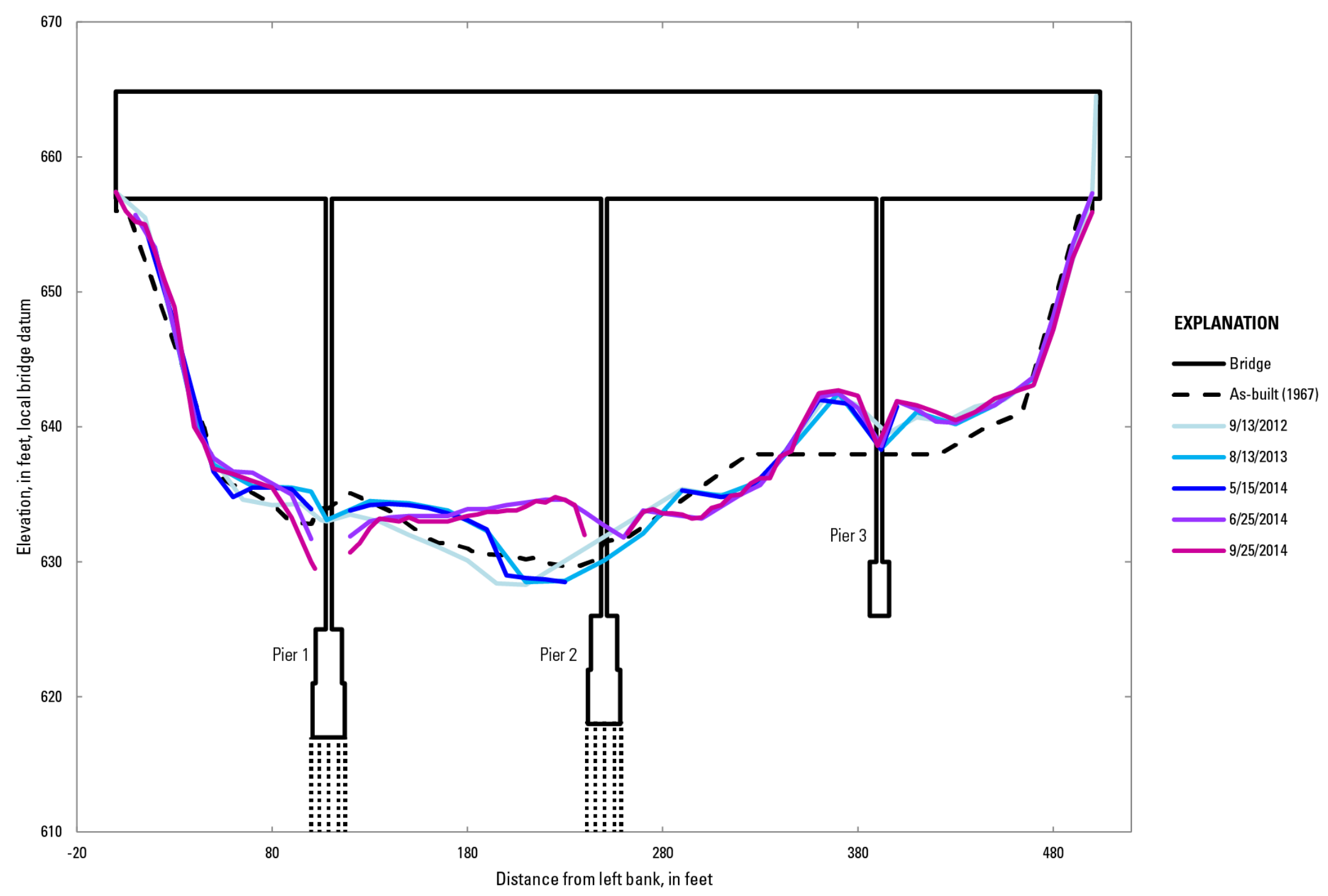

Figure 50. Cross sections showing upstream soundings at bridge 527, Salcha River, Alaska, 2012-14. 
BN 527 Salcha River, upstream

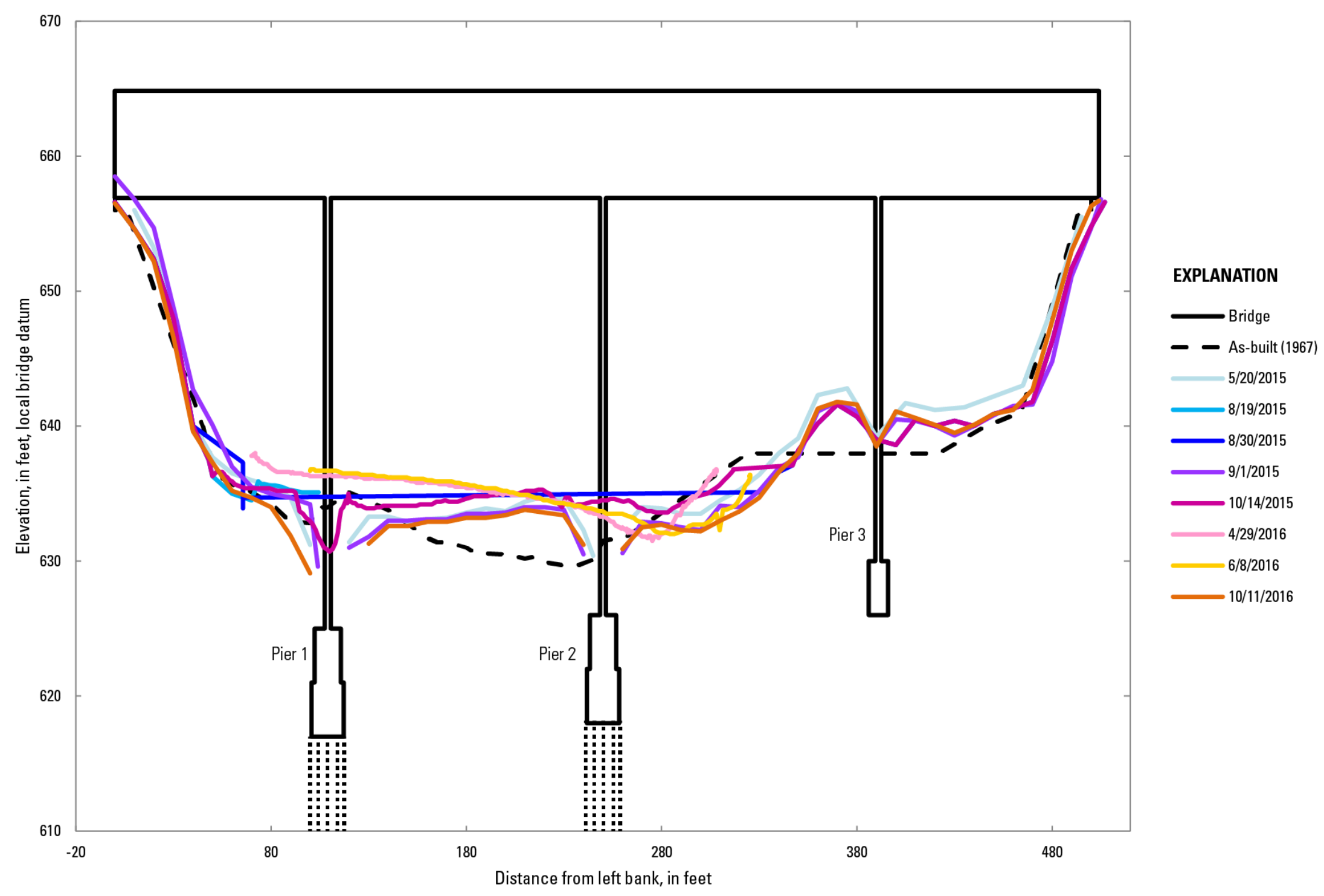

Figure 51. Cross sections showing upstream soundings at bridge 527, Salcha River, Alaska, 2015-16. 


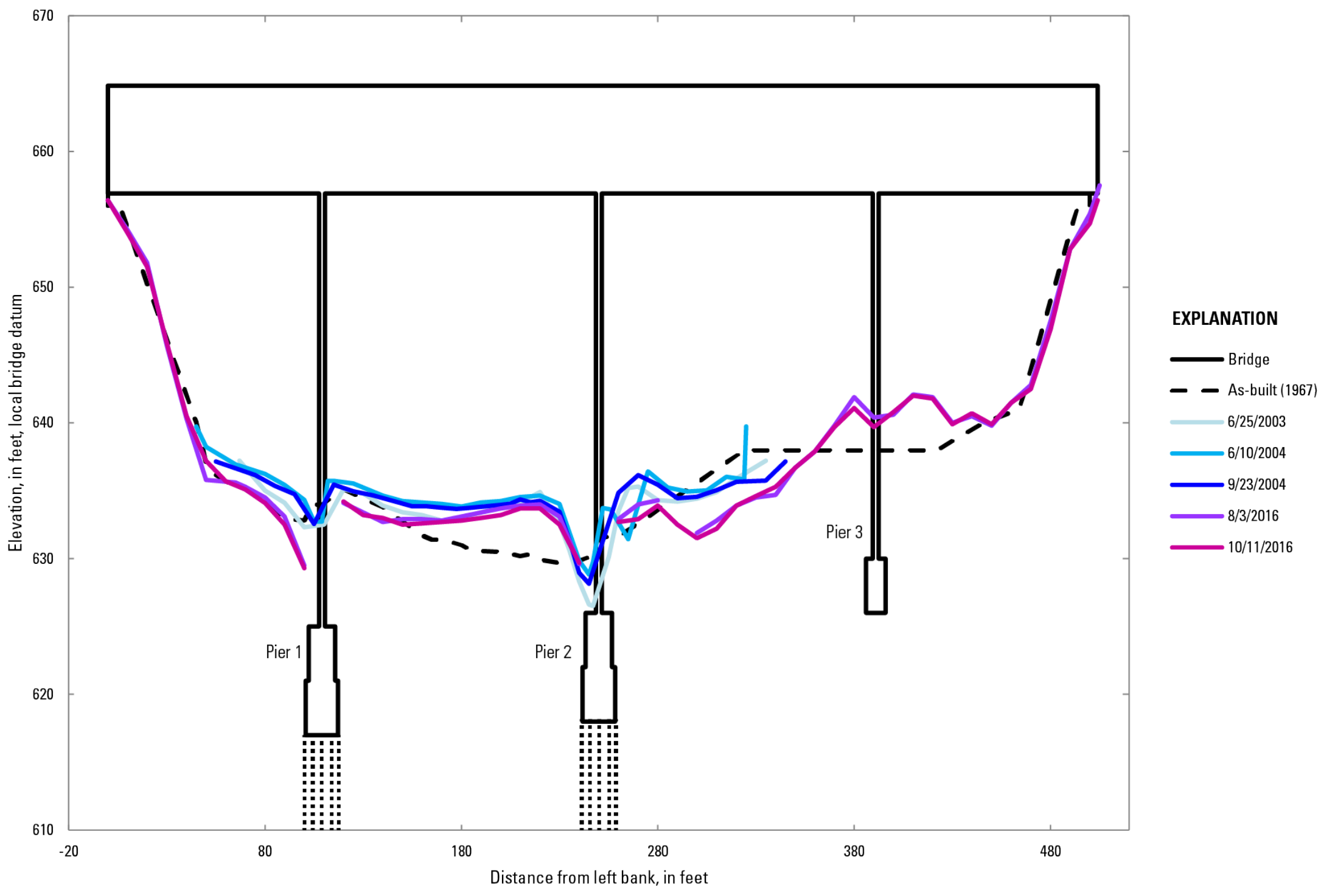

Figure 52. Cross sections showing downstream soundings at bridge 527, Salcha River, Alaska, 2003-16. 


\section{Bridge 539, Knik River*}

The Knik River is a braided, sand-and-gravel channel that transports large quantities of sediment from the Knik Glacier, which is about 6 mi upstream. Pier-mounted sonar data record an annual pattern of channel aggradation and degradation punctuated by shorter periods of scour and fill related to high flows and the complex pier geometry (Conaway, 2007). Large annual changes in bed elevation across the channel are partially attributed to the nearly 4-to-1 channel contraction at the bridge during high flows. An older bridge and piers directly upstream of the bridge add to the complexity and extent of the scour. Other factors influencing scour at this site are the upstream guide banks and changes in sediment supply. Substantial scour has occurred during every year of monitoring from 2005 to 2013 and 2014 to 2016 (figs. 53-57). Conaway (2006) noted a pattern of increased scour during warm phases of the Pacific Decadal Oscillation due to increased discharge from glacial melt. At higher flows, scour occurs as flow is routed along both guide banks, although in more recent years increasingly more scour has been observed along the right bank. The most substantial scour (as much as $13 \mathrm{ft}$ ) tends to occur during July-August, whereas data collected in spring and later in autumn show that bed elevations across the channel return to about the same elevation of those in the as-built cross section. Scour reaches critical levels when the footing and sub-footing of the right bank pier are exposed by the cumulative effects of local scour at the pier and contraction scour. 
BN 539 Knik River, upstream

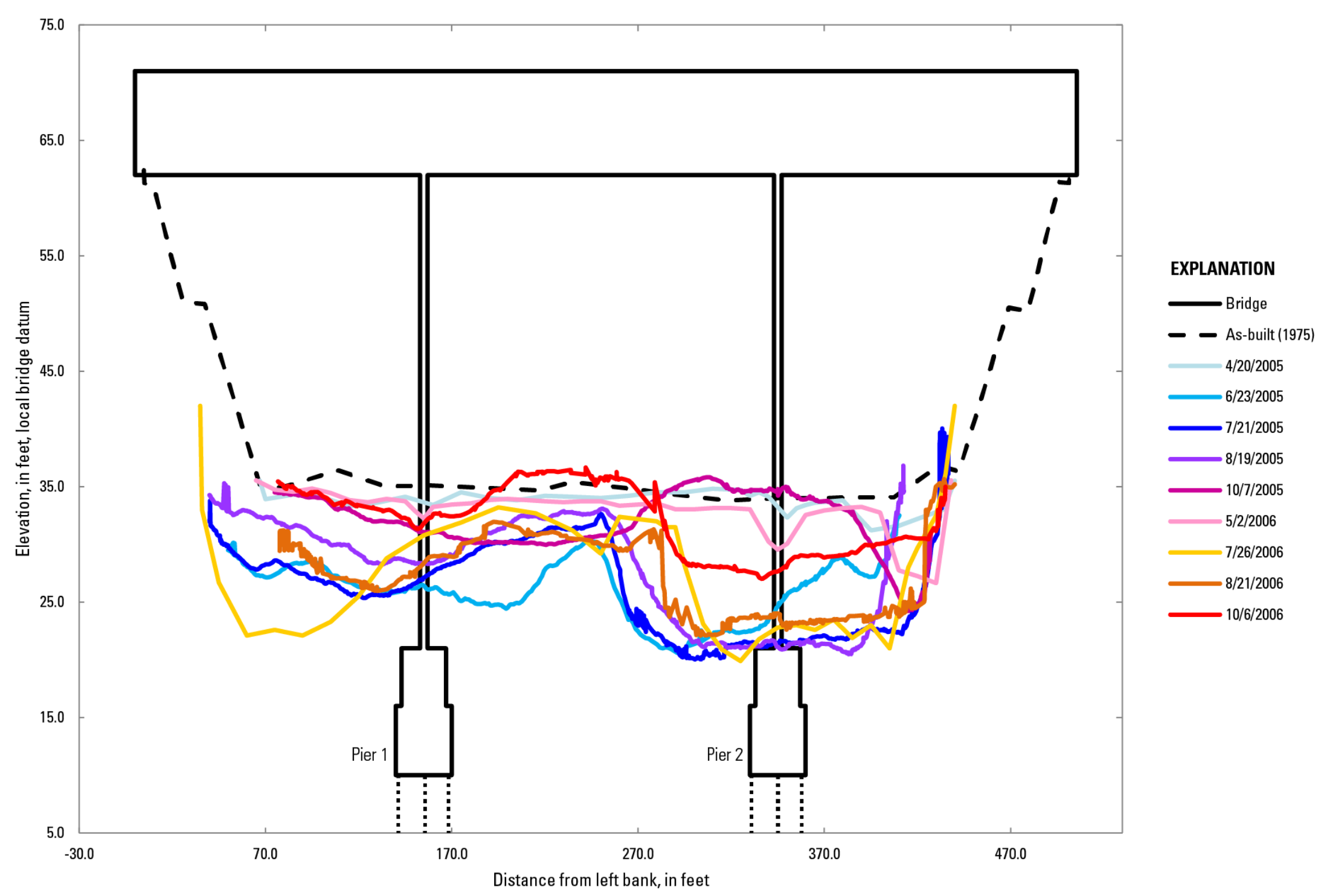

Figure 53. Cross sections showing upstream soundings at bridge 539, Knik River, Alaska, 2005-06. 
BN 539 Knik River, upstream

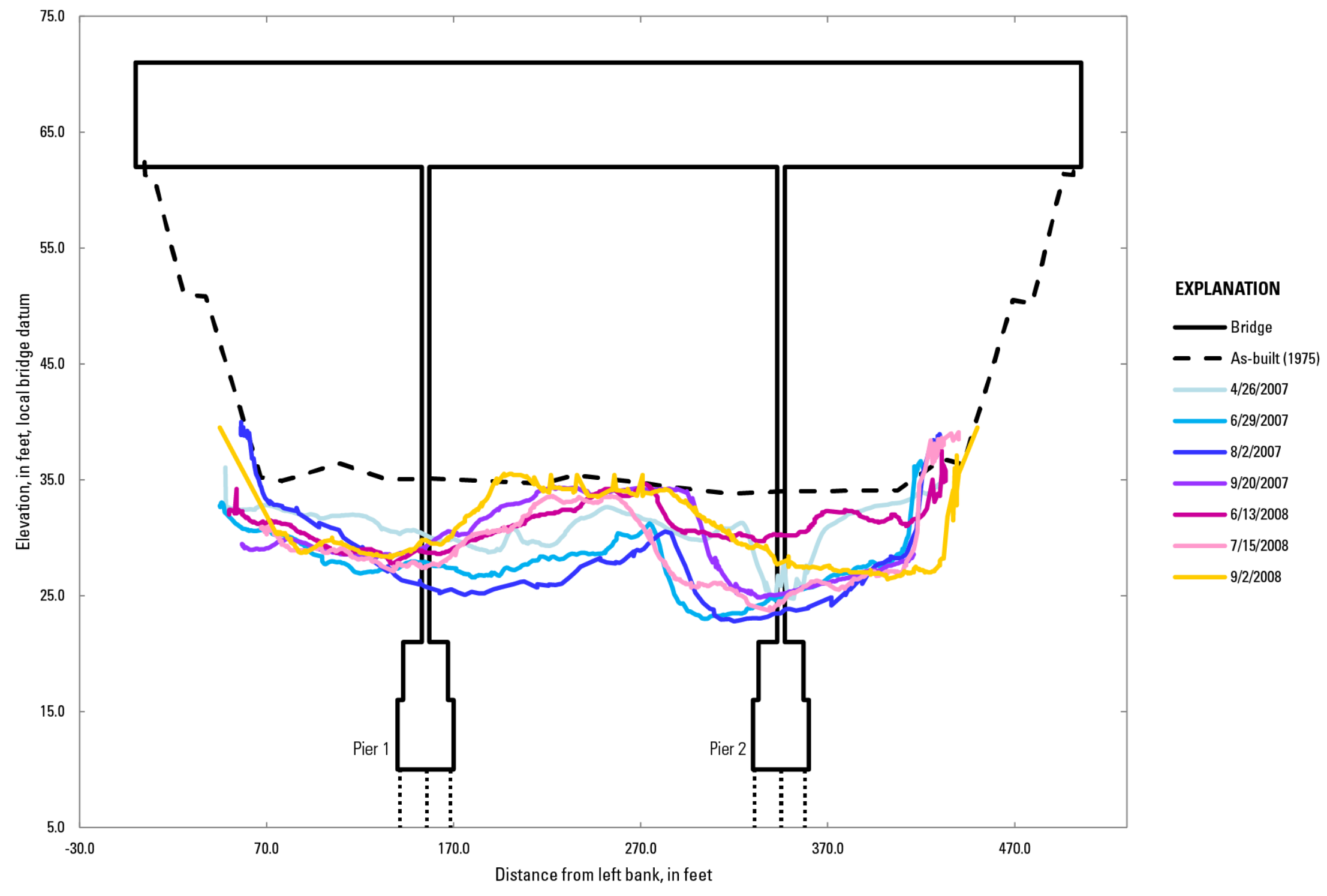

Figure 54. Cross sections showing upstream soundings at bridge 539 Knik River, Alaska, 2007-08. 
BN 539 Knik River, upstream

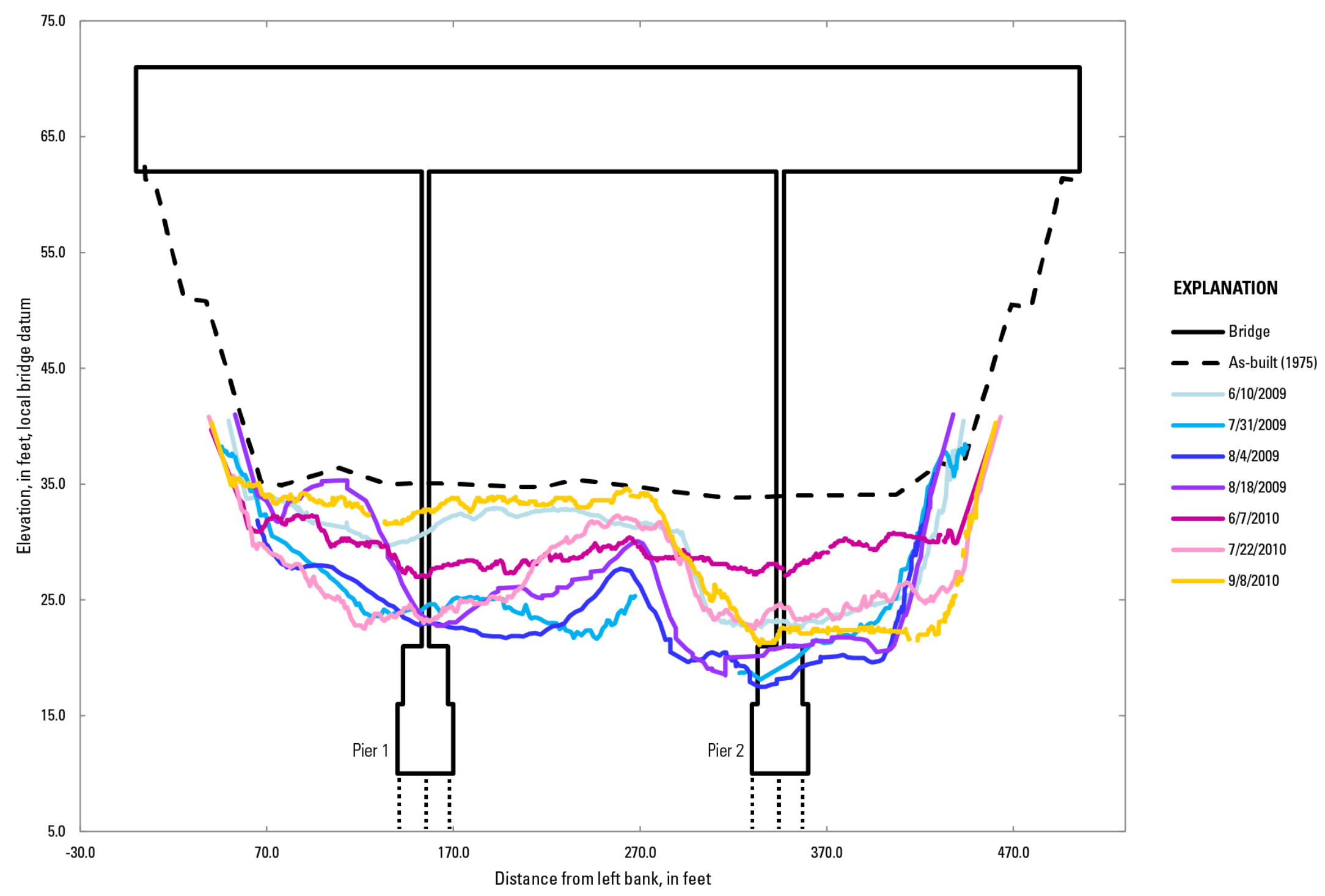

Figure 55. Cross sections showing upstream soundings at bridge 539, Knik River, Alaska, 2009-10. 
BN 539 Knik River, upstream

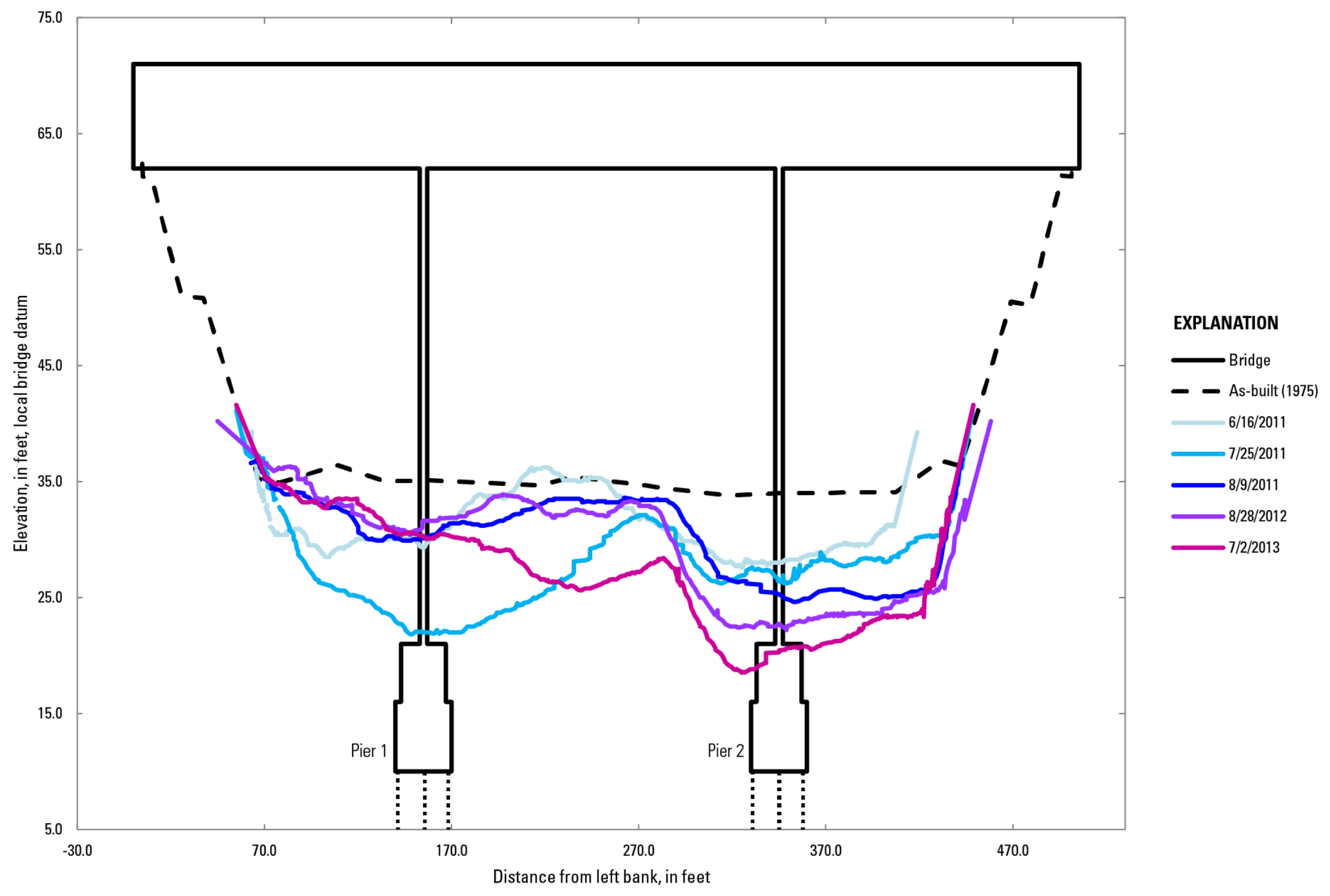

Figure 56. Cross sections showing upstream soundings at bridge 539, Knik River, Alaska, 2011-13. 
BN 539 Knik River, upstream

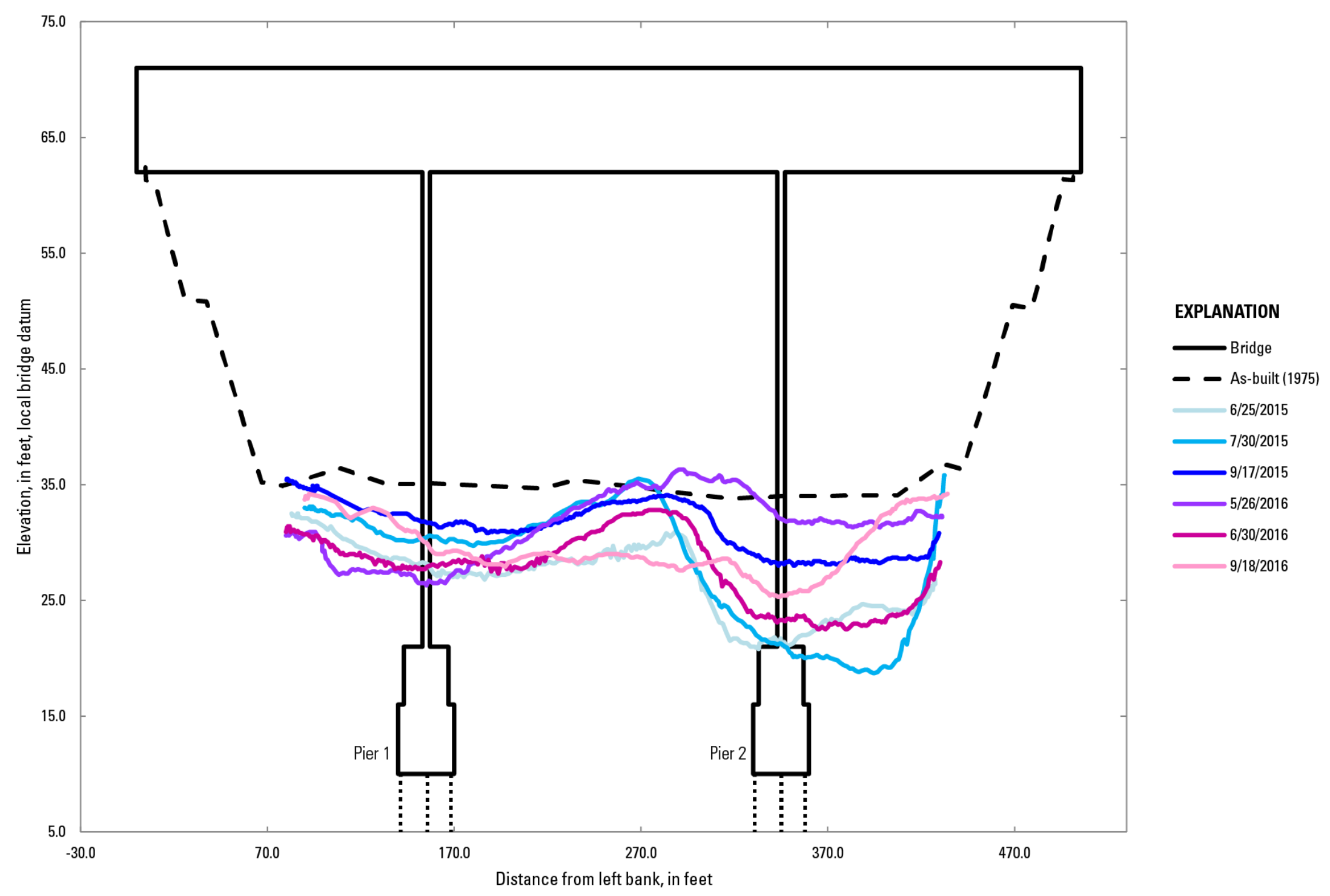

Figure 57. Cross sections showing upstream soundings at bridge 539, Knik River, Alaska, 2015-16. 


\section{Bridge 573, Tazlina River*}

The Tazlina River, draining from glacially fed Tazlina Lake, is a tributary of the Copper River. The right pier, the channel to the right of the pier, and both banks are extensively armored with riprap as a countermeasure to scour. Guide banks on both sides direct flow through the bridge. The river makes a slight bend to the left through the bridge and most of the flow is between the right pier and the right bank. Outburst floods occur every 1-3 years from one or more of four lakes that form behind the Tazlina River by the Tazlina and Nelchina Glaciers. The outburst floods typically are the highest peak discharges at the site and have the greatest effect on channel change.

Cross sections were collected along the upstream side of the bridge during 1997, 1999, 2002-07, 2012, 2013, 2015, and 2016 (figs. 58-61). Maximum streambed scour of $14 \mathrm{ft}$ was observed in the right side of the channel. The remainder of the channel has aggraded $6 \mathrm{ft}$ since the as-built survey. Flow velocities greater than $13 \mathrm{ft} / \mathrm{s}$ have prohibited the measurement of accurate streambed depths in the right side of the channel for the past 3 years (2013-16). 
BN 573 Tazlina River, upstream

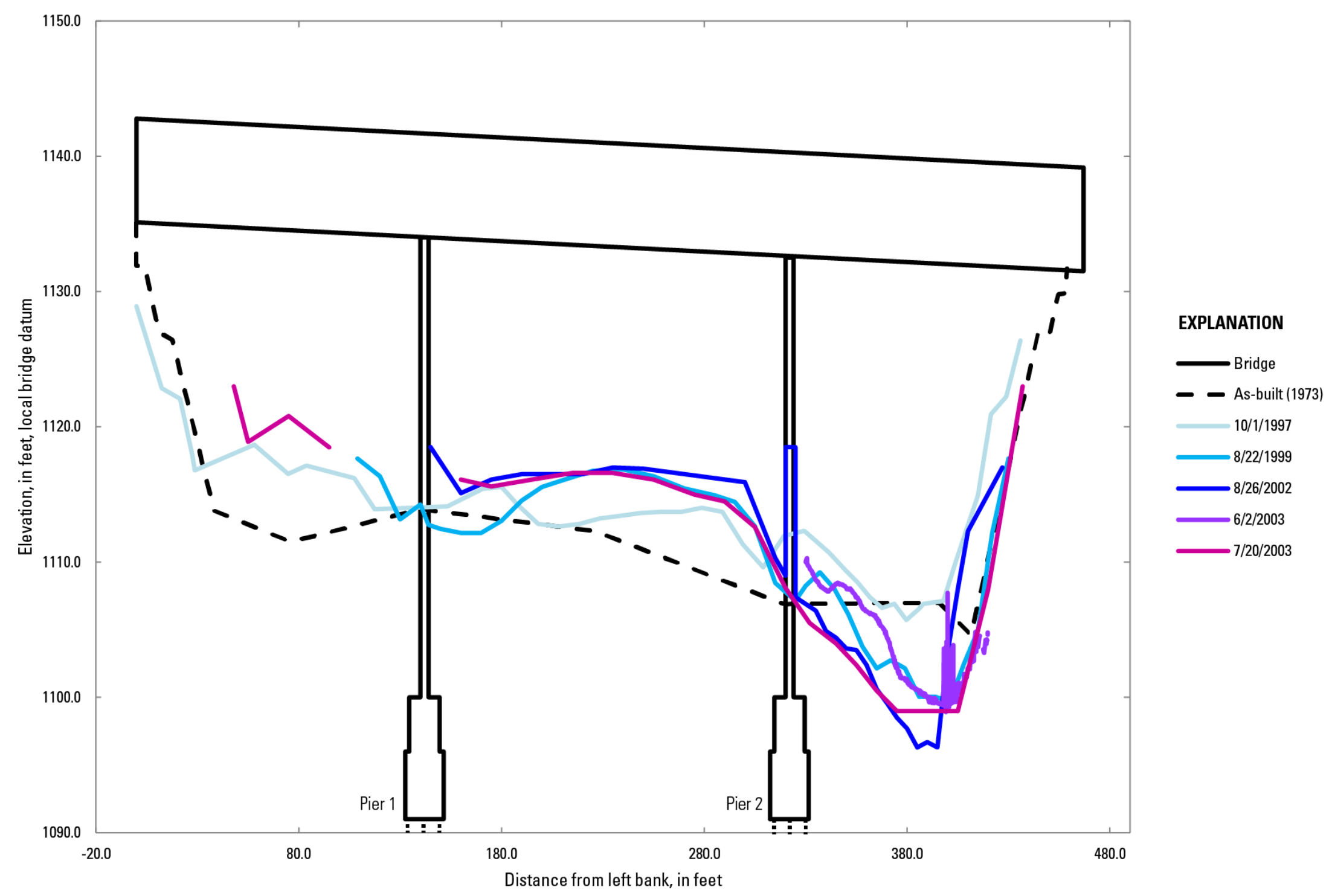

Figure 58. Cross sections showing upstream soundings at bridge 573, Tazlina River, Alaska, 1997-2003. 
BN 573 Tazlina River, upstream

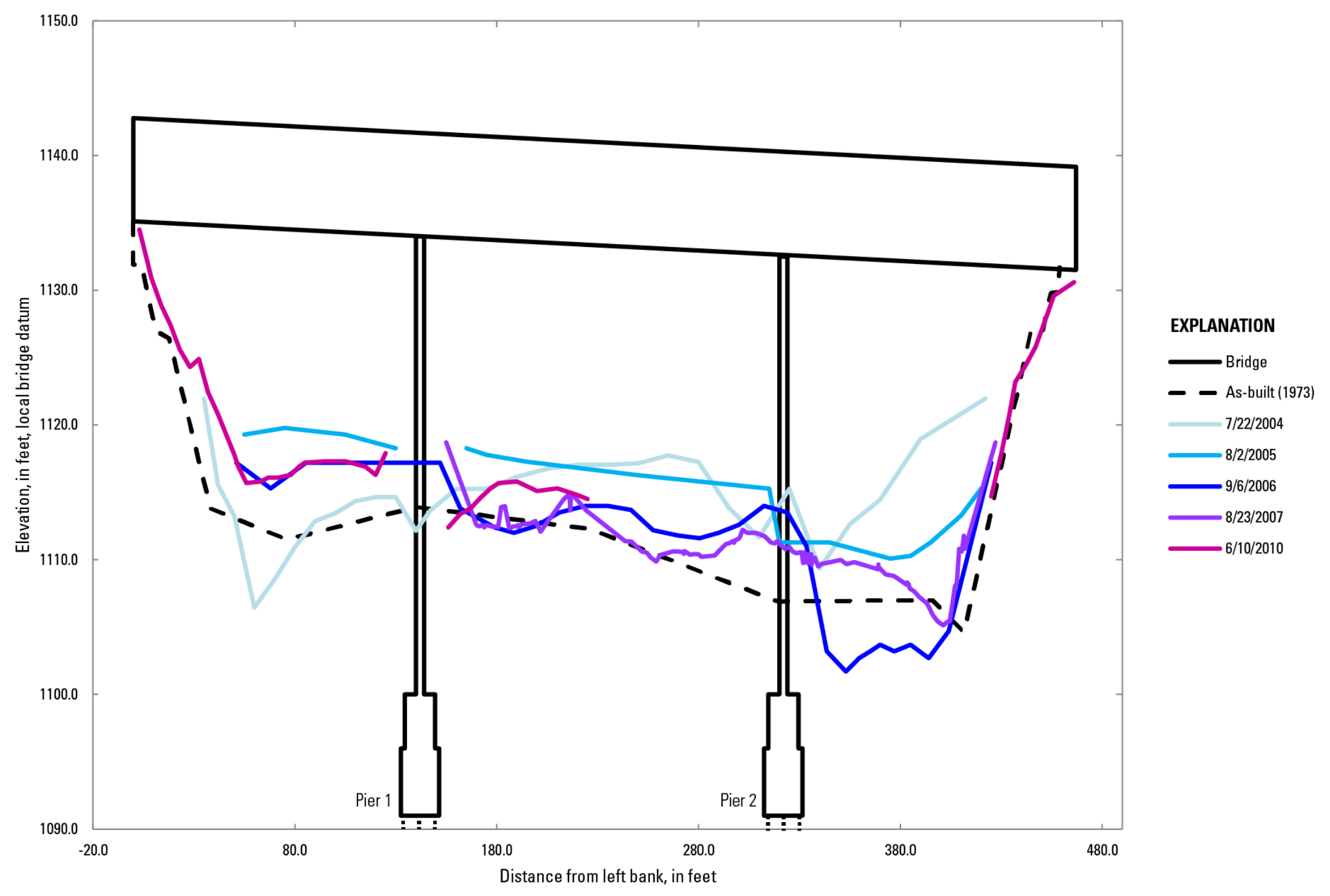

Figure 59. Cross sections showing upstream soundings at bridge 573, Tazlina River, Alaska, 2004-10. 
BN 573 Tazlina River, upstream

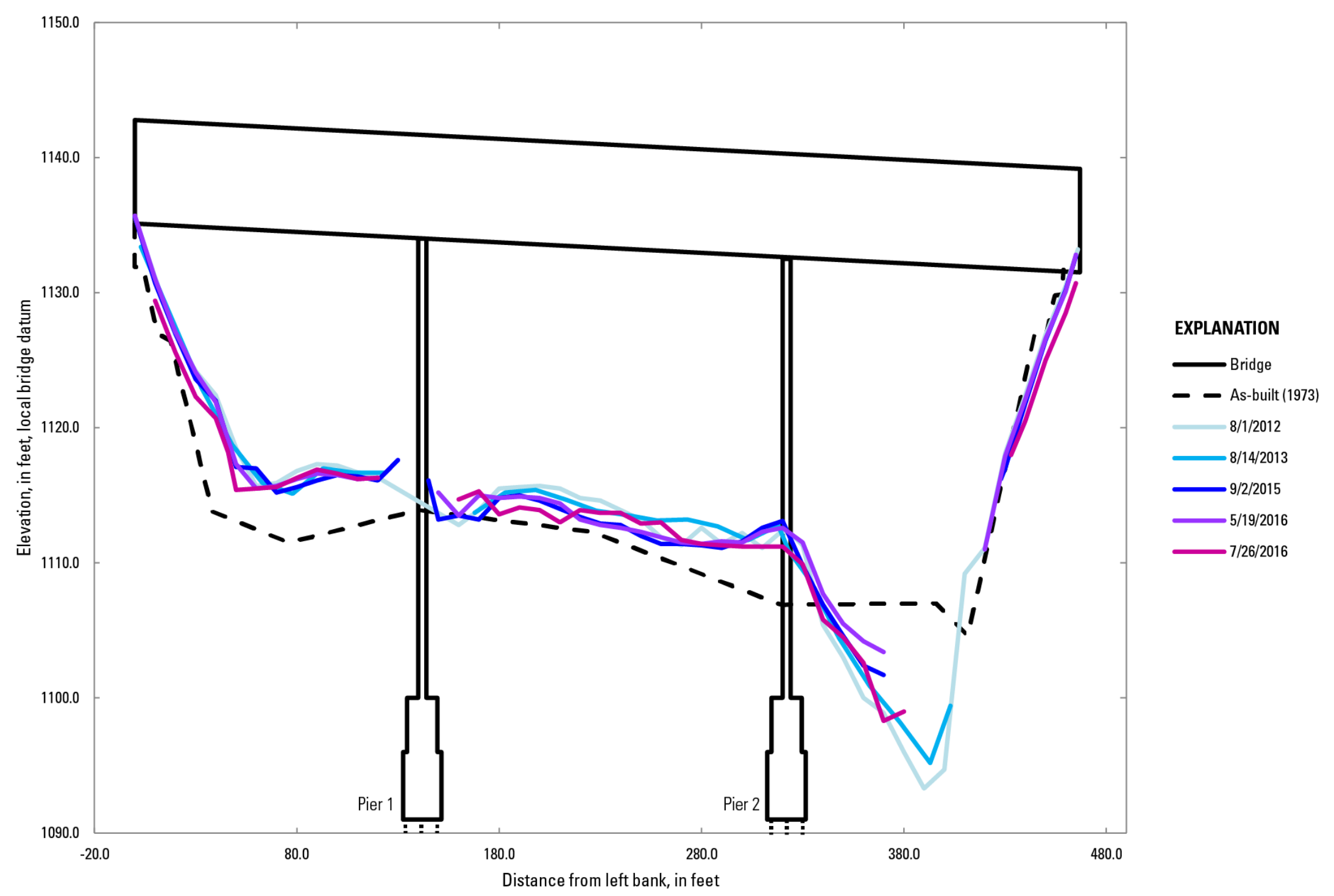

Figure 60. Cross sections showing upstream soundings at bridge 573, Tazlina River, Alaska, 2012-16. 
BN 573 Tazlina River, downstream

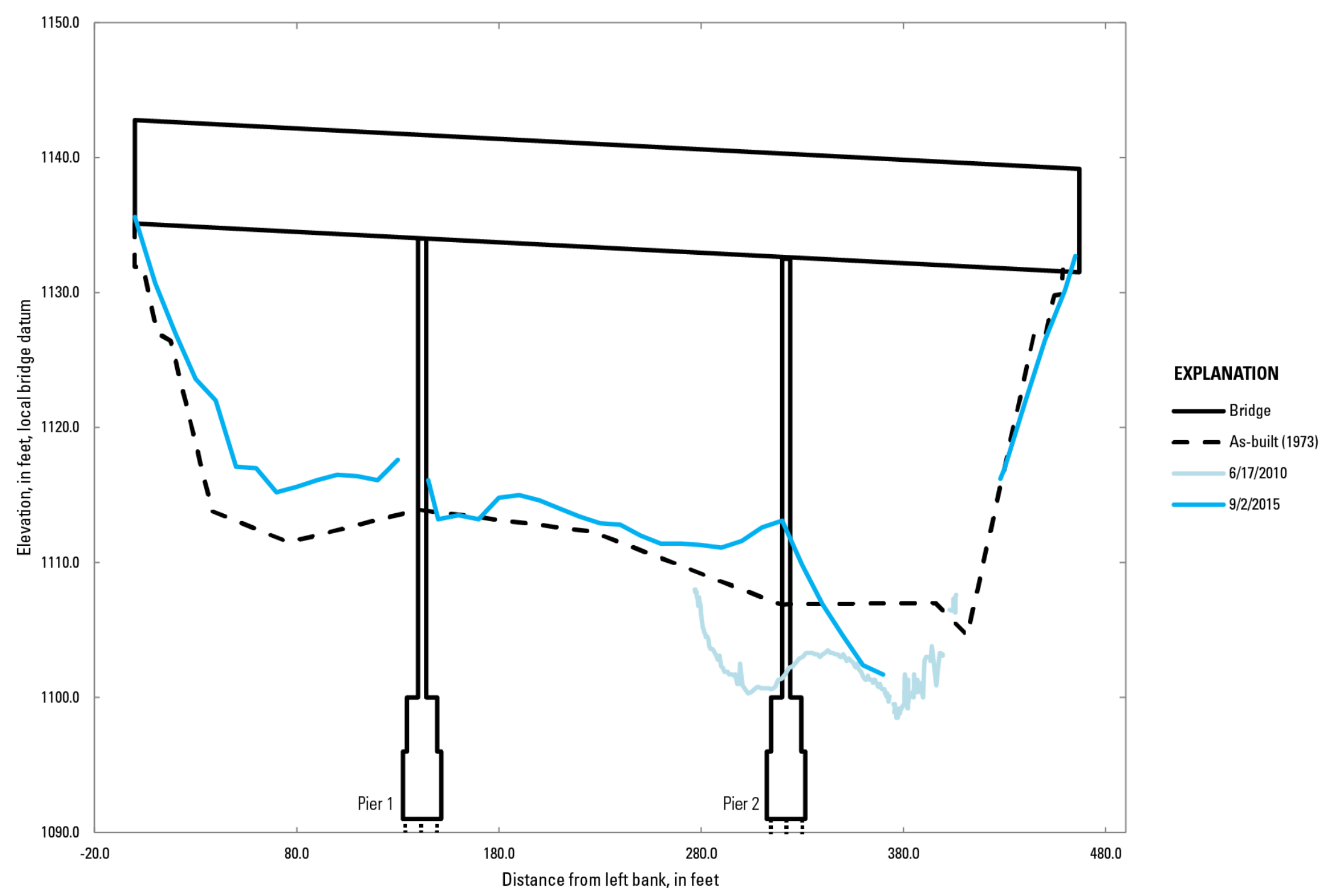

Figure 61. Cross sections showing downstream soundings at bridge 573, Tazlina River, Alaska, 2010-15. 


\section{Bridge 654, Slana River*}

The Slana River is a tributary of the Copper River, originating in the Alaska Range. It is a single meandering channel near the bridge. Cross sections were collected from 2002 to 2011 (fig. 62). The asbuilt cross section indicates a low streambed near the left most pier. Soundings indicate that an additional $3 \mathrm{ft}$ of local scour occurred around the left pier, whereas as much as $3 \mathrm{ft}$ of aggradation occurred across the right side of the channel. 
BN 654 Slana River, upstream

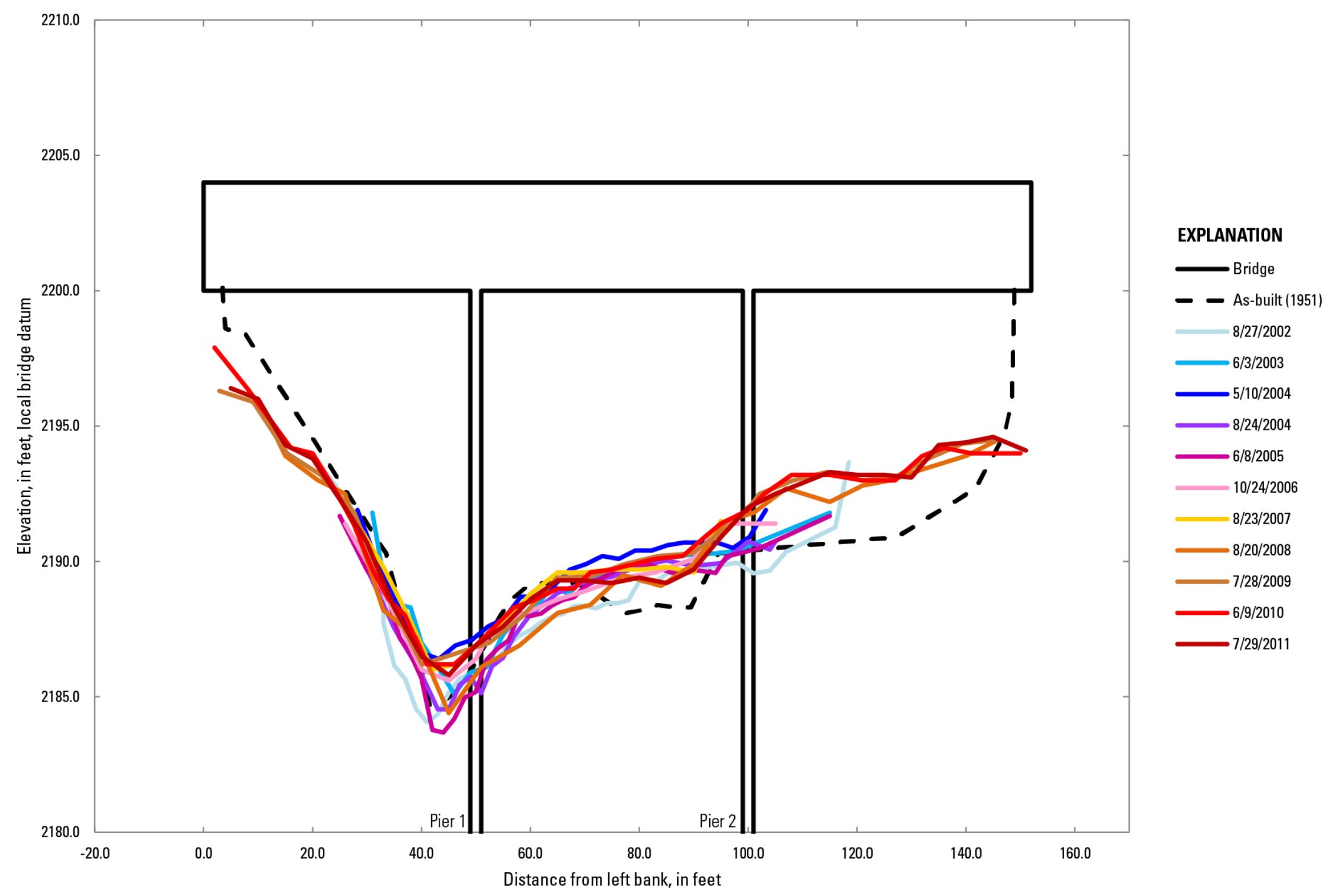

Figure 62. Cross sections showing upstream soundings at bridge 654, Slana River, Alaska, 2002-11. 
Bridge 663, Tok River*

The Tok River is a wide, braided gravel-bed river originating in the Alaska Range and is a tributary of the Tanana River. Cross sections were measured in 2002 and from 2004 until the bridge was replaced in 2014 (figs. 63 and 64). The channel had an overall aggradation of $5 \mathrm{ft}$ relative to the as-built cross-section. From 2004 to 2005, $6 \mathrm{ft}$ of localized pier scour occurred on the rightmost pier, and from 2013 to 2014, $6 \mathrm{ft}$ of scour occurred on the leftmost pier. 
BN 663 Tok River, upstream

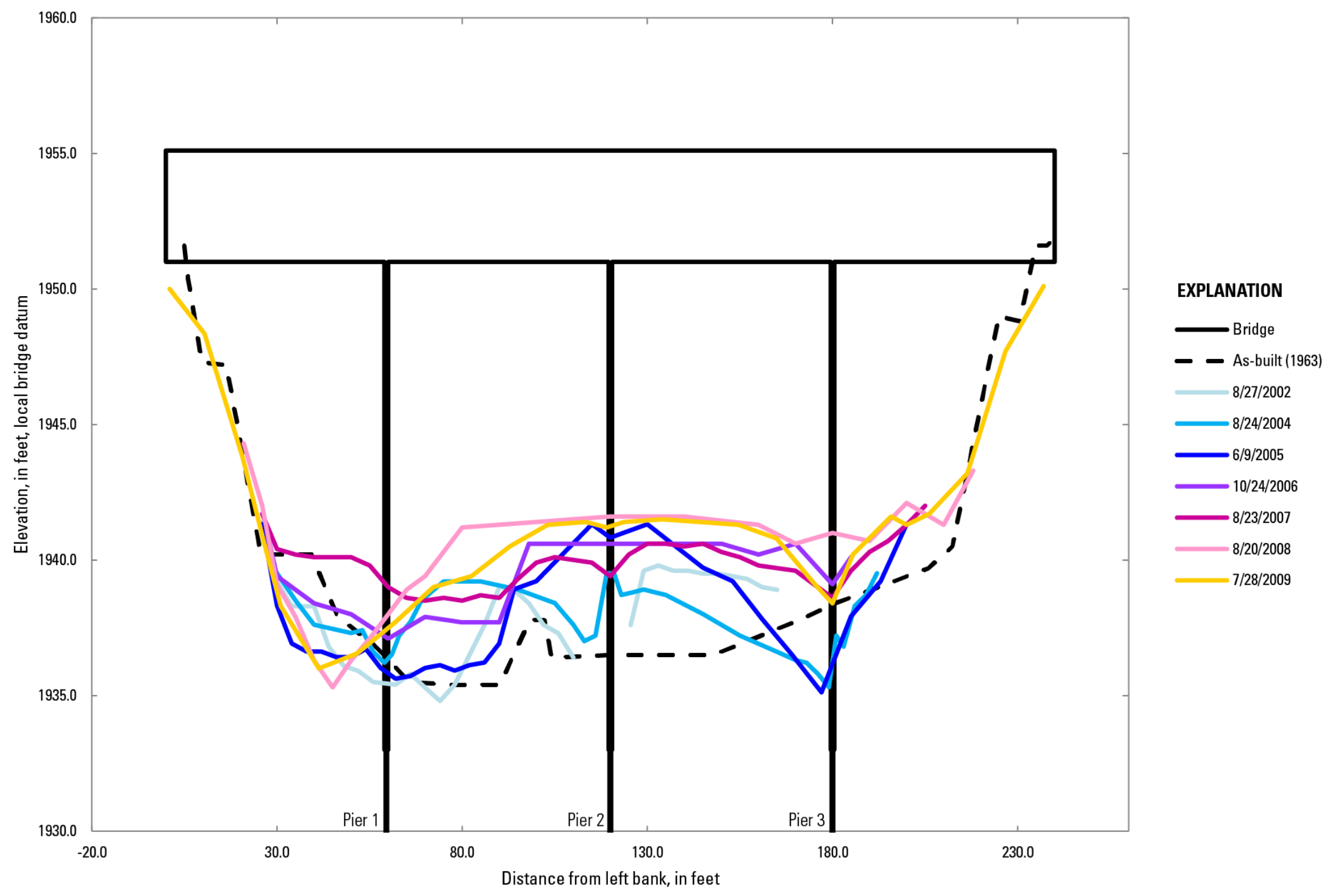

Figure 63. Cross sections showing upstream soundings at bridge 663, Tok River, Alaska, 2002-09. 
BN 663 Tok River, upstream

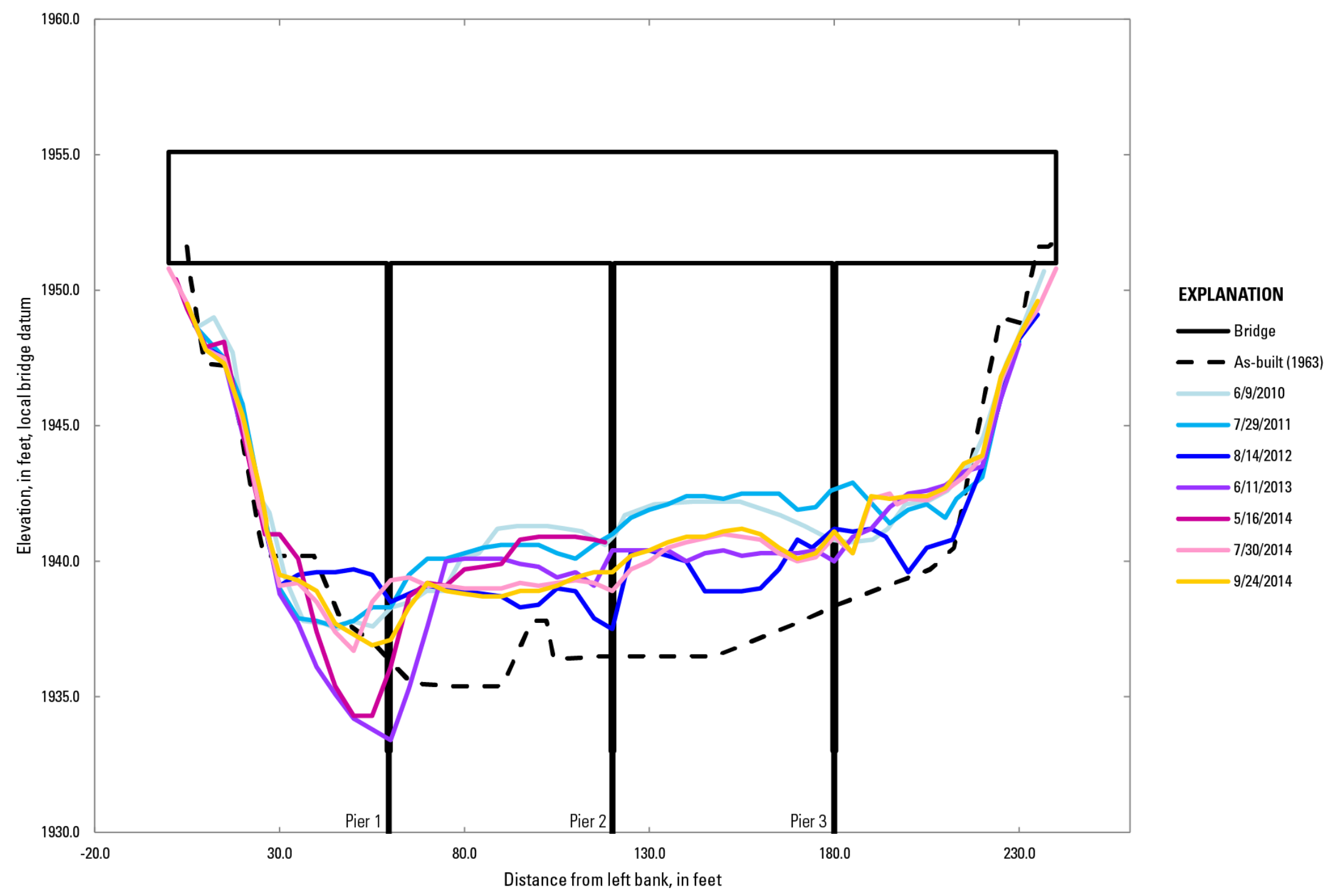

Figure 64. Cross sections showing upstream soundings at bridge 663, Tok River, Alaska, 2010-14. 


\section{Bridge 670, Kasilof River*}

The Kasilof River is a silt-rich river that originates in Tustumena Lake, a large proglacial lake draining the Kenai Mountains. The lake naturally attenuates flow in the Kasilof River; discharge slowly increases throughout the summer, typically peaking between late July and mid-August, and then slowly decreases before freeze-up unless large autumn rainstorms maintain high lake levels. Cross sections were measured during 2002, 2004, 2005, and 2007-16 (figs. 65-67). At bridge 670, the channel generally is stable with very little change occurring in streambed elevation since monitoring first began. However, relative to the as-built, as much as $6 \mathrm{ft}$ of degradation occurred on the right side of the channel. During the first few years of monitoring (2002-05), $6 \mathrm{ft}$ of localized scour occurred around the rightmost pier. In 2004, soundings at the right-bank pier indicated that the pier footing was exposed. 
BN 670 Kasilof River, upstream

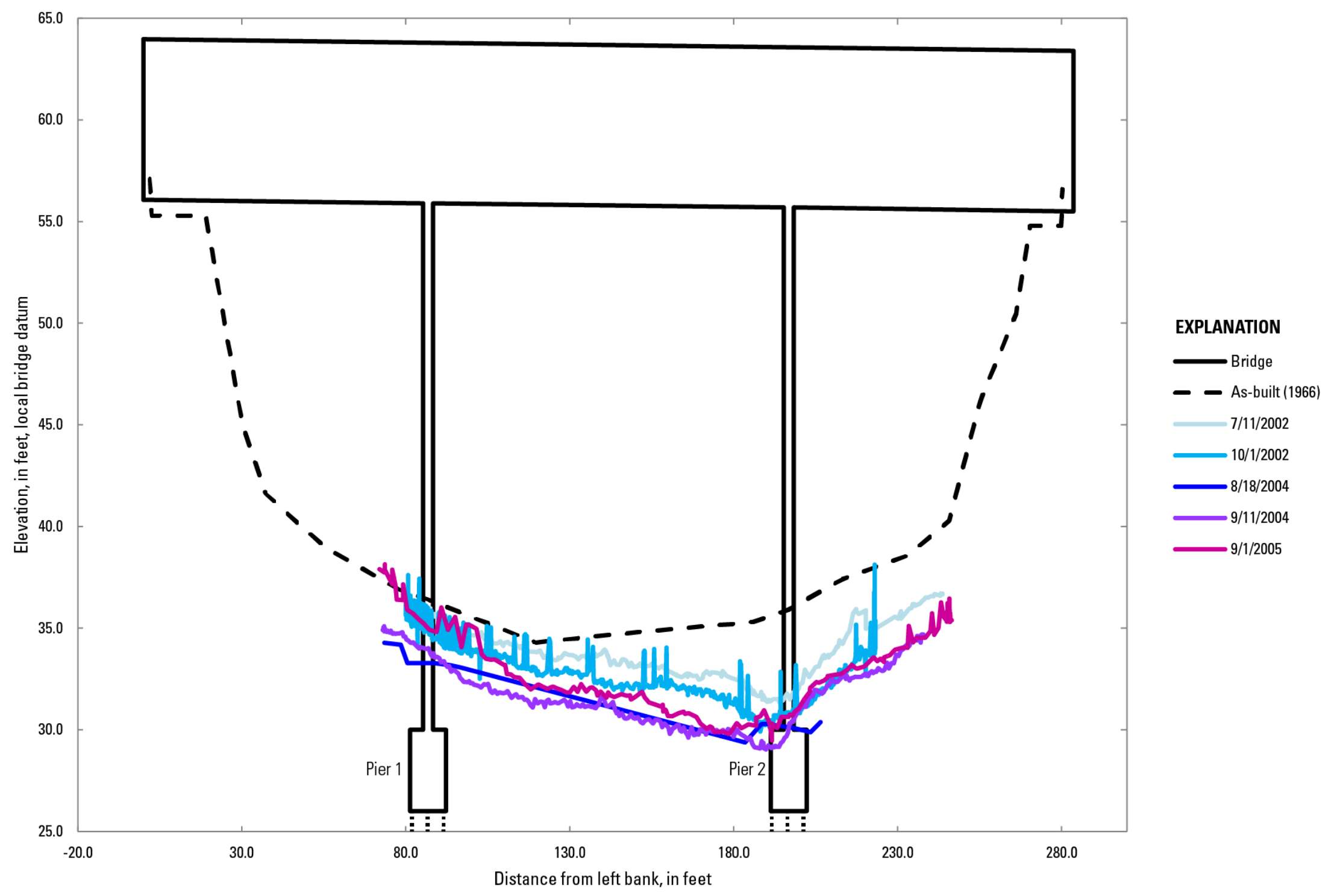

Figure 65. Cross sections showing upstream soundings at bridge 670, Kasilof River, Alaska, 2002-05. 
BN 670 Kasilof River, upstream

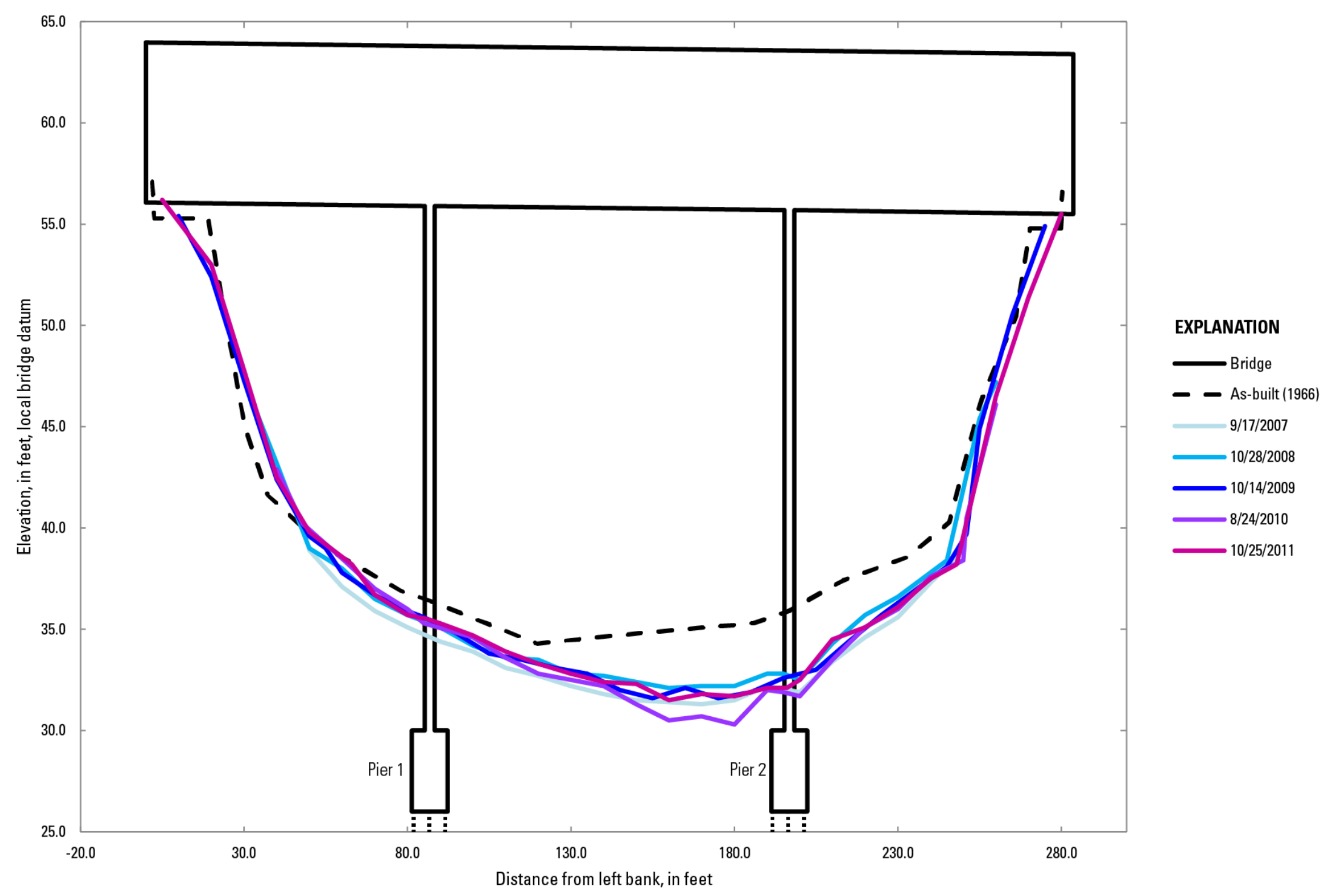

Figure 66. Cross sections showing upstream soundings at bridge 670, Kasilof River, Alaska, 2007-11. 
BN 670 Kasilof River, upstream

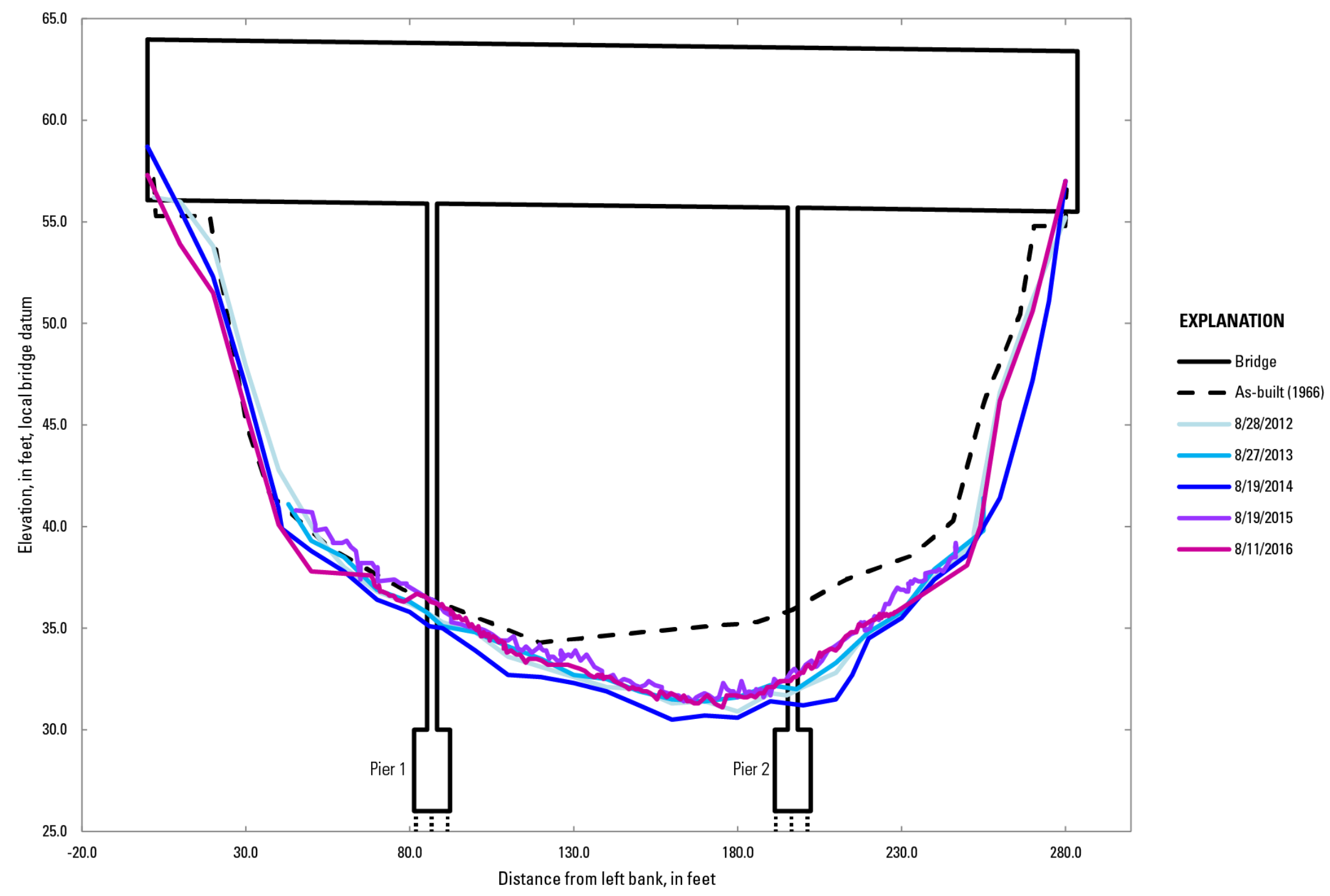

Figure 67. Cross sections showing upstream soundings at bridge 670, Kasilof River, Alaska, 2012-16. 
Bridge 742, Chilkat River*

The Chilkat River originates at Chilkat Glacier in the Coast Range on the border of Alaska and British Columbia. The river flows in a single channel through the bridge, which crosses about $1 \mathrm{mi}$ upstream of the Klehini River confluence. Cross sections were measured during 2001, 2004-11, and 2013-16 (figs. 68-70). Since the as-built survey, $7 \mathrm{ft}$ of aggradation occurred on the left bank with little to no degradation on the right bank. From 2006 to 2010, the Chilkat River scoured as much as 7 feet locally around piers 2, 5, and 6 (pier numbering starts from the left side). From 2013 to 2016, as much as $8 \mathrm{ft}$ of scour occurred between piers 2 and 4, and aggradation of as much as $6 \mathrm{ft}$ occurred around the other piers. 
BN 742 Chilkat River, upstream

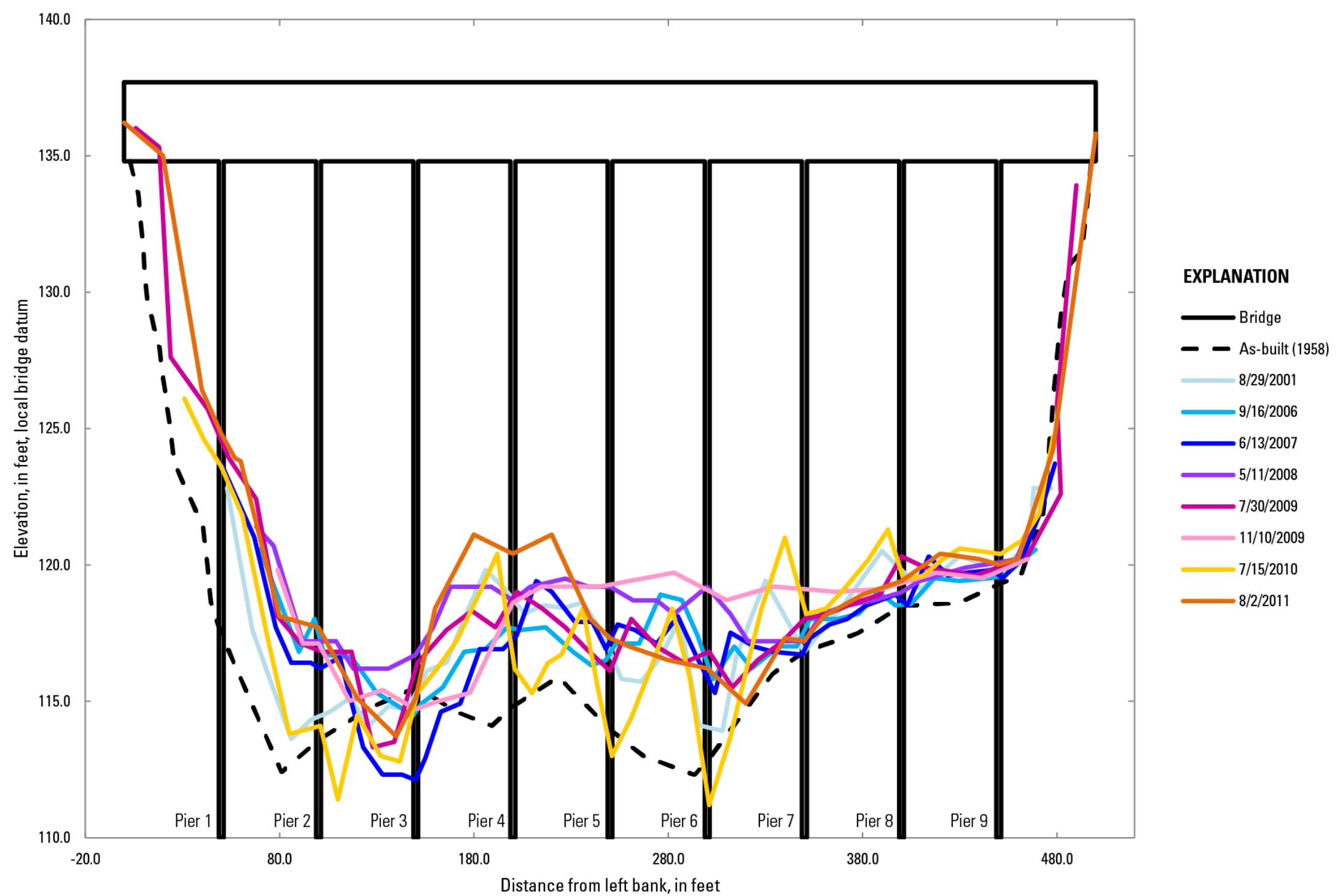

Figure 68. Cross sections showing upstream soundings at bridge 742, Chilkat River, Alaska, 2001-11. 
BN 742 Chilkat River, upstream

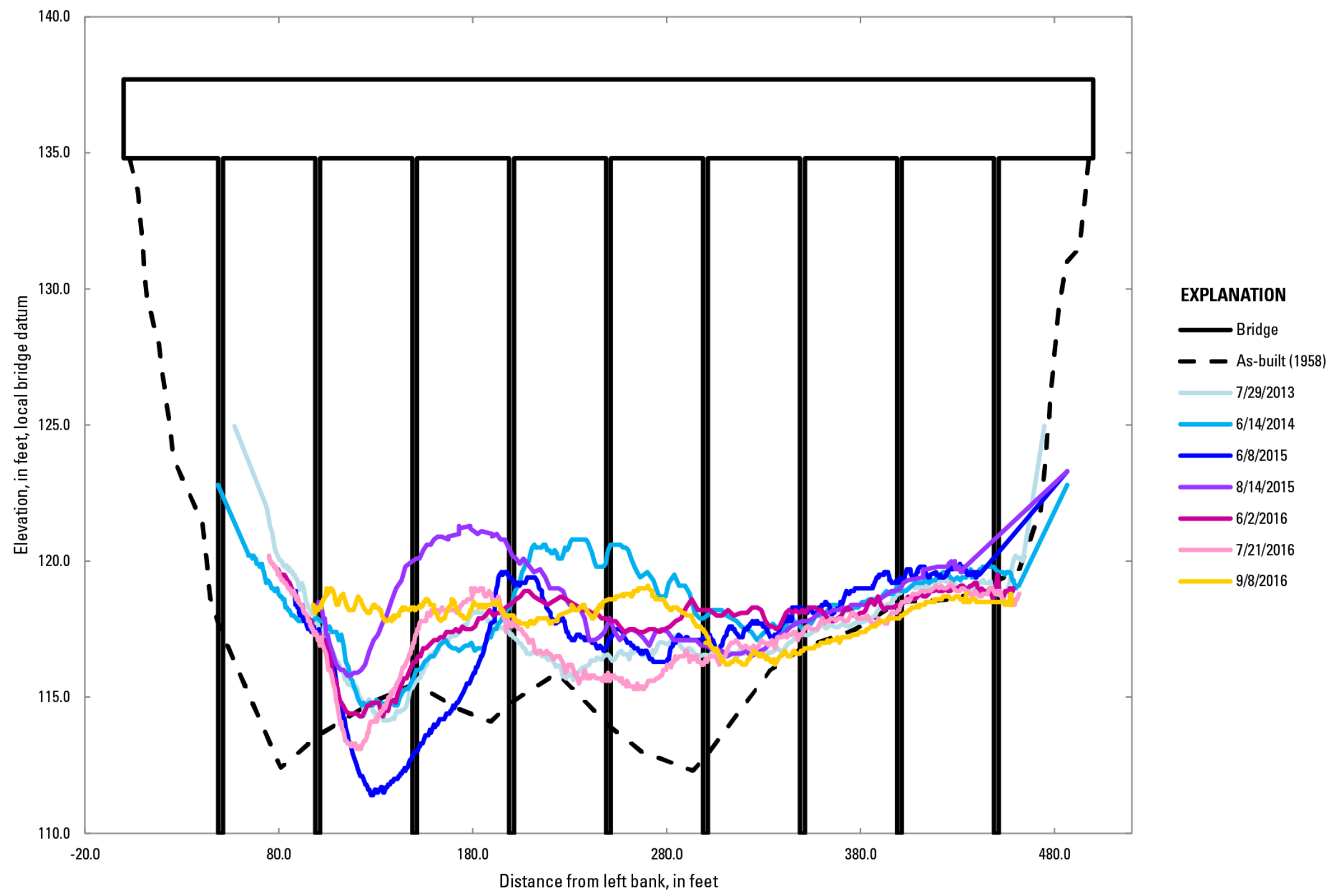

Figure 69. Cross sections showing upstream soundings at bridge 742, Chilkat River, Alaska, 2013-16. 
BN 742 Chilkat River, downstream

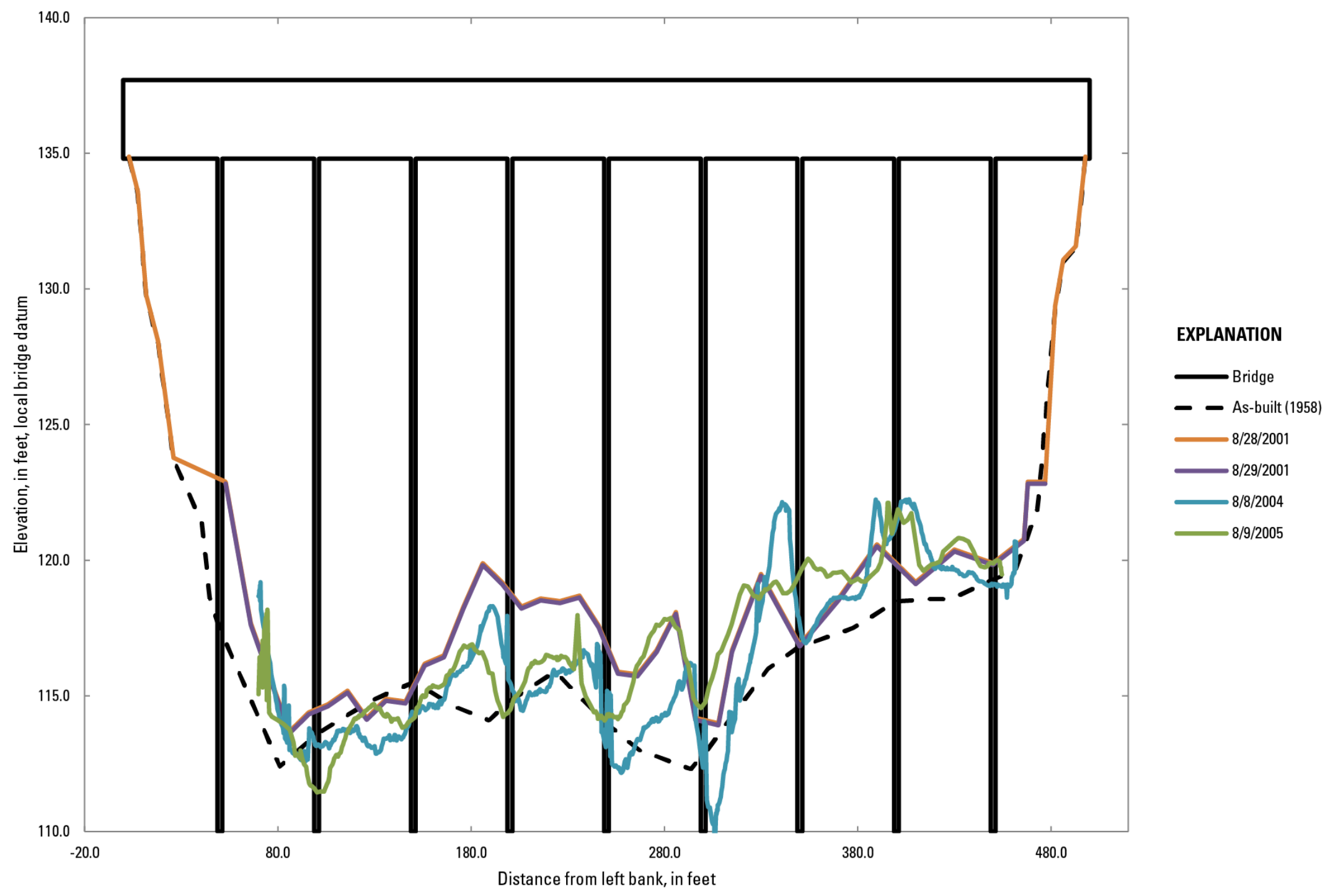

Figure 70. Cross sections showing downstream soundings at bridge 742, Chilkat River, Alaska, 2001-05. 


\section{Bridge 857, Nenana River at Healy}

The Nenana River is a tributary of the Tanana River that originates at the Nenana Glacier on the southern side of the Alaska Range. Bridge 857 is about $500 \mathrm{ft}$ downstream of a railroad bridge. During low-to-moderate flows, gravel bars are present upstream of the bridge and extend upstream of the railroad bridge dividing the approach flow into two separate channels. During high flows, there are no exposed gravel bars and standing waves are present throughout the reach. Cross sections were measured during 1999, 2000, 2003-09, 2011, and 2015 (figs. 71-74). The rightmost pier is subject to debris accumulation. Most of the flow has been confined to the left side of the channel since the bridge was constructed, and pier footings were exposed in this part of the channel in all the soundings. Most of the scour (as much as $4 \mathrm{ft}$ ) has been confined between the first and second piers. On the right side of the channel, aggradation of as much as $4 \mathrm{ft}$ has occurred between the second and third pier. 
BN 857 Nenana River at Healy, downstream

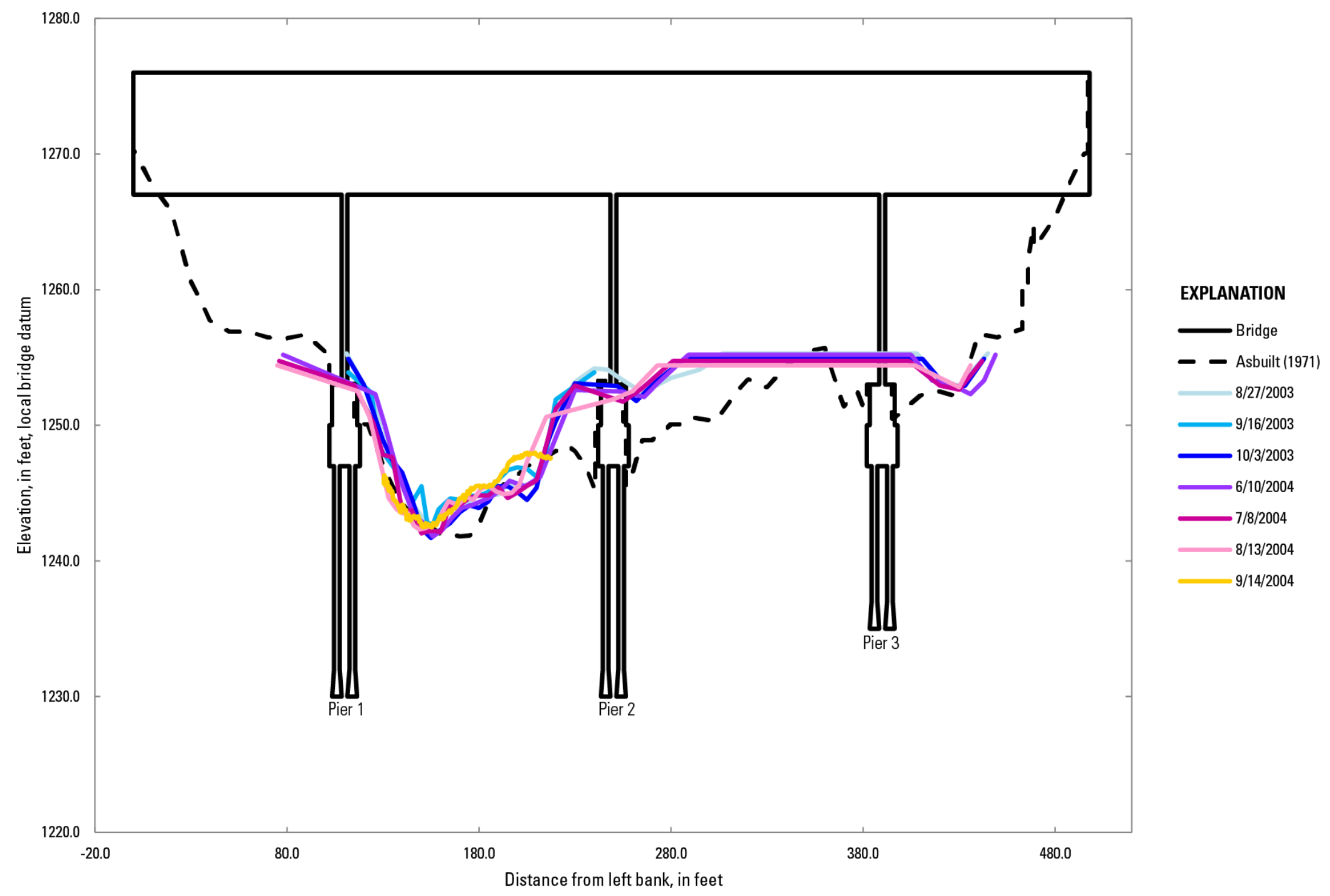

Figure 71. Cross sections showing downstream soundings at bridge 857, Nenana River at Healy, Alaska, 2003-04. 
BN 857 Nenana River at Healy, downstream

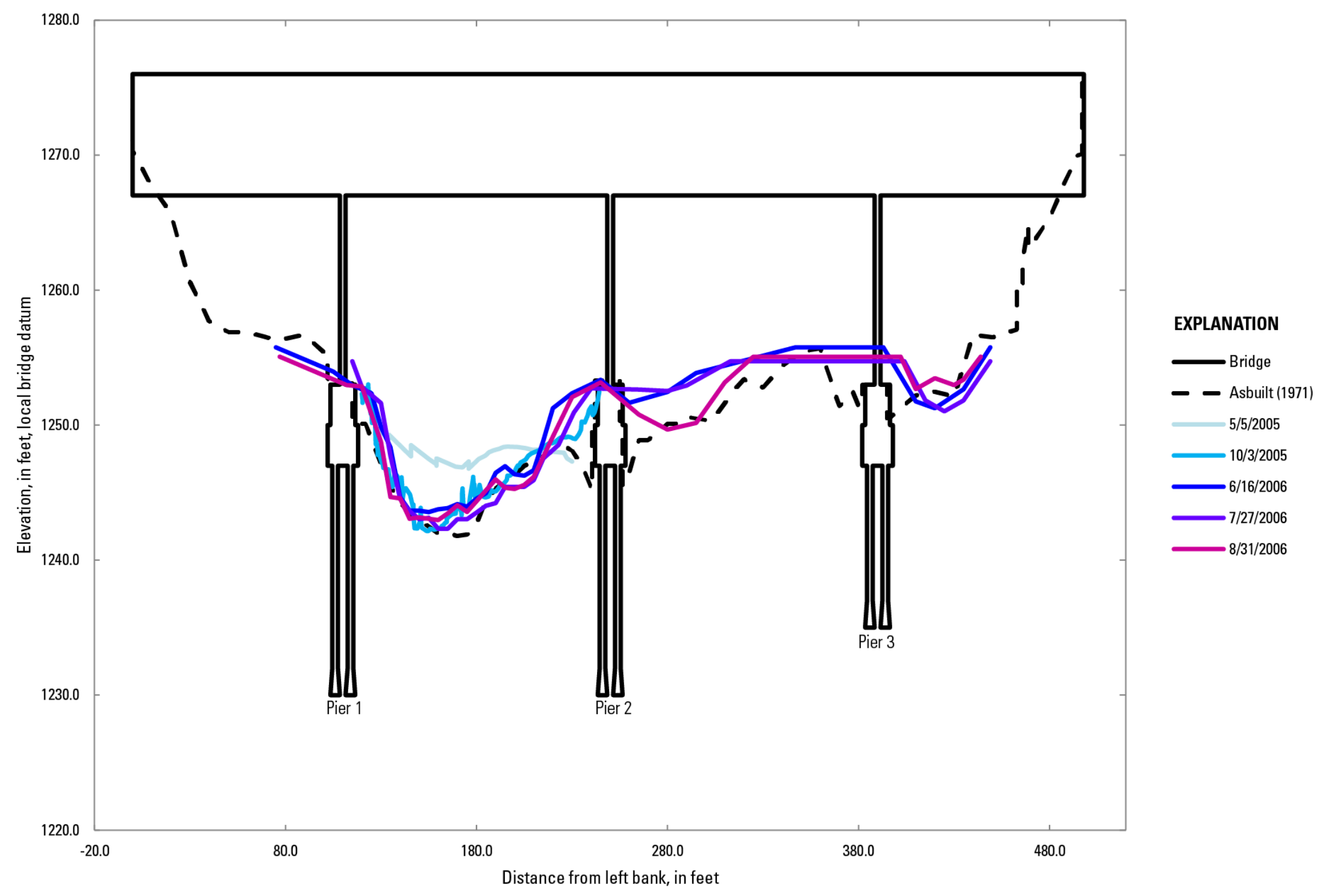

Figure 72. Cross sections showing downstream soundings at bridge 857, Nenana River at Healy, Alaska, 2005-06. 
BN 857 Nenana River at Healy, downstream

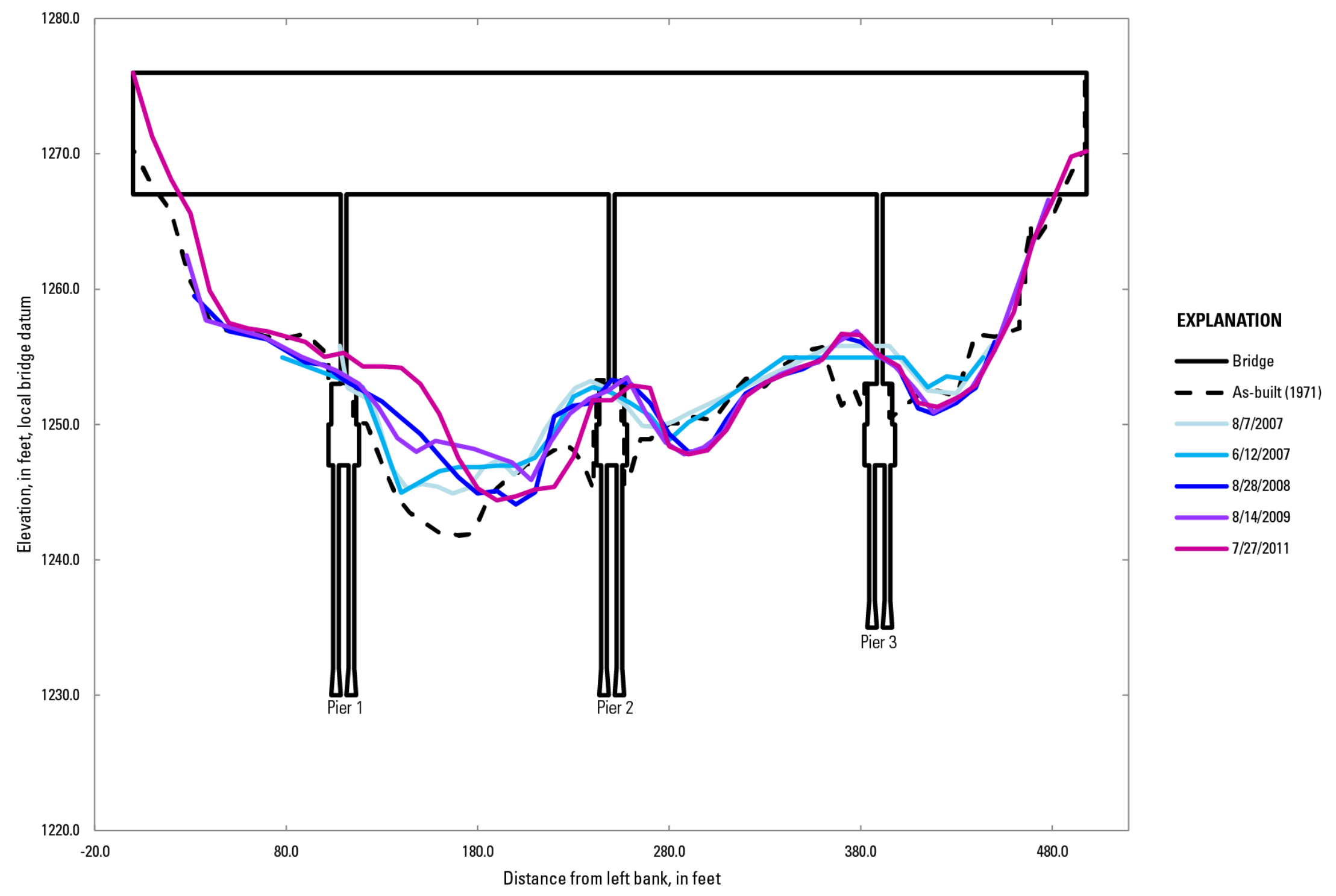

Figure 73. Cross sections showing downstream soundings at bridge 857, Nenana River at Healy, Alaska, 2007-11. 
BN 857 Nenana River at Healy, upstream

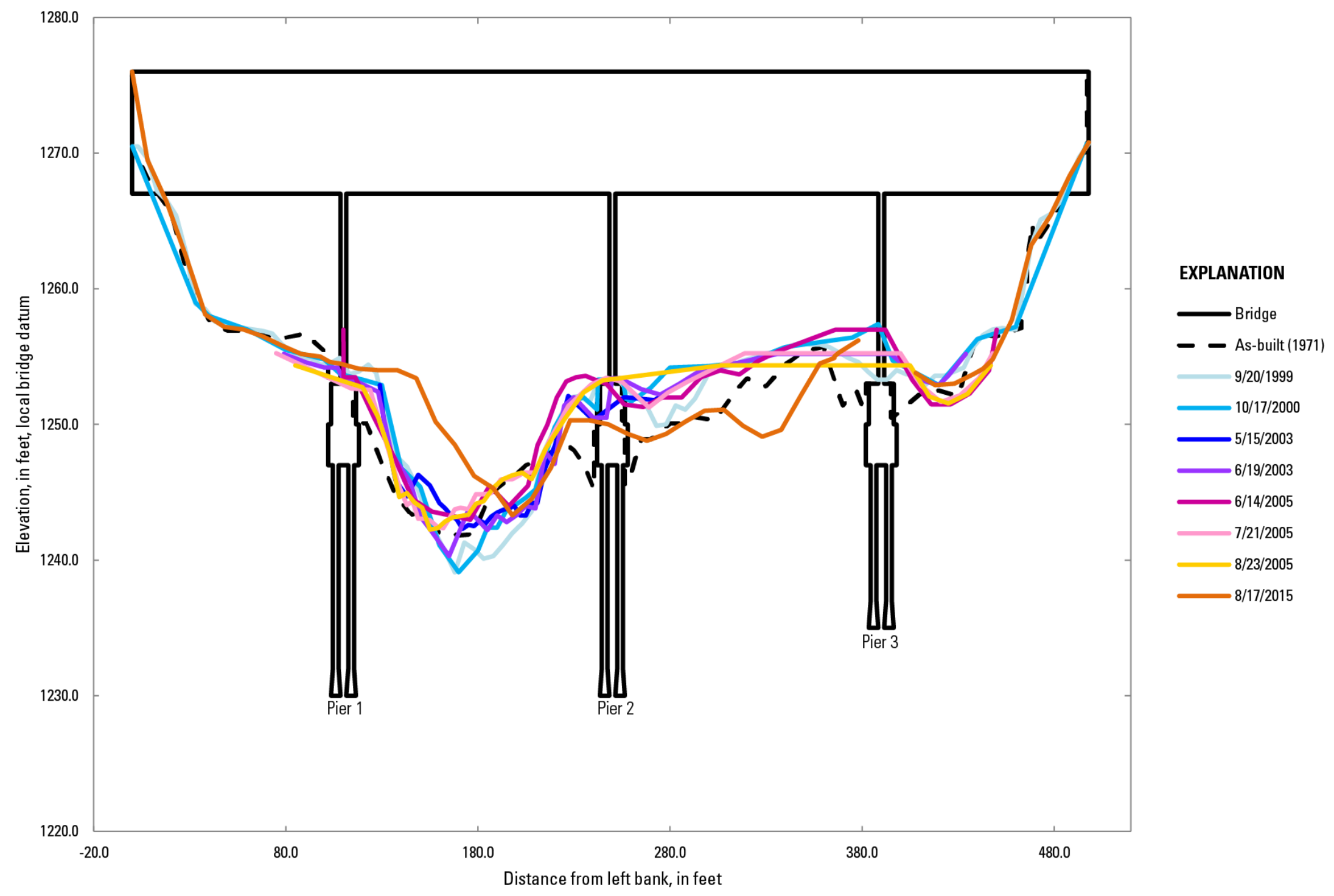

Figure 74. Cross sections showing upstream soundings at bridge 857, Nenana River at Healy, Alaska, 1999-2015. 


\section{Bridge 983, Red Cloud River*}

Red Cloud River is a steep mountain stream that originates on the eastern side of Sharatin Mountain on Kodiak Island. Directly upstream of the bridge is the confluence of the Red Cloud River and an unnamed creek that drains from the mountains on the southeastern side of the Red Cloud Basin. Red Cloud River is a cobble-rich river that is prone to flooding during heavy rains. Changes in discharge are rapid at this site owing to the small drainage area, high gradient of the stream, and intense precipitation. Cross sections were measured during 2005-11 and 2013-16 (figs. 75-78). Relative to the as-built cross section, about $3 \mathrm{ft}$ of aggradation occurred across the channel. Soundings also indicate continuous lateral channel migration through the bridge. As much as $3 \mathrm{ft}$ of local scour at the pier occurred since 2014 , likely aggravated by debris lodged on the pier. 
BN 983 Red Cloud River, upstream

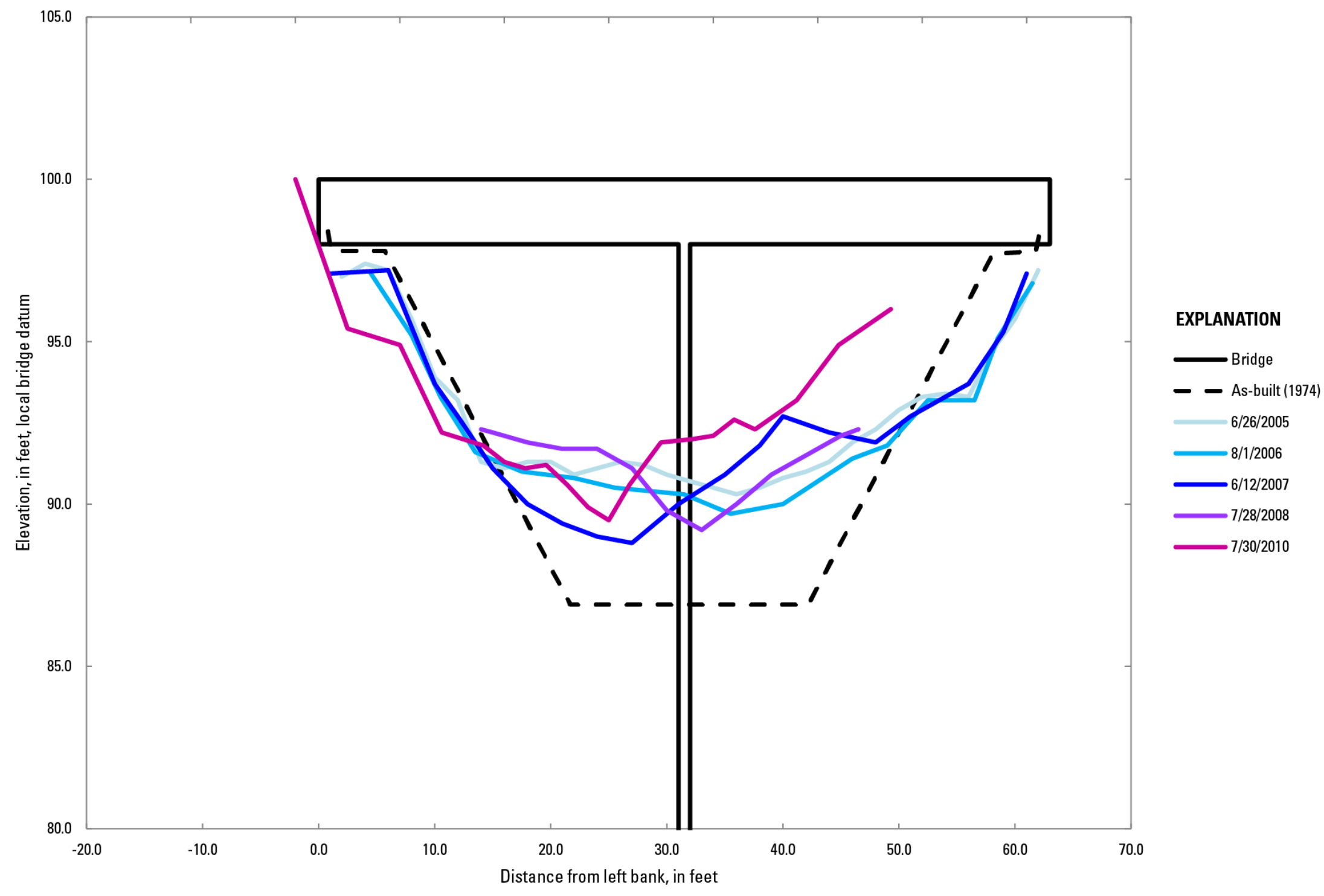

Figure 75. Cross sections showing upstream soundings at bridge 983, Red Cloud River, Alaska, 2005-10. 
BN 983 Red Cloud River, upstream

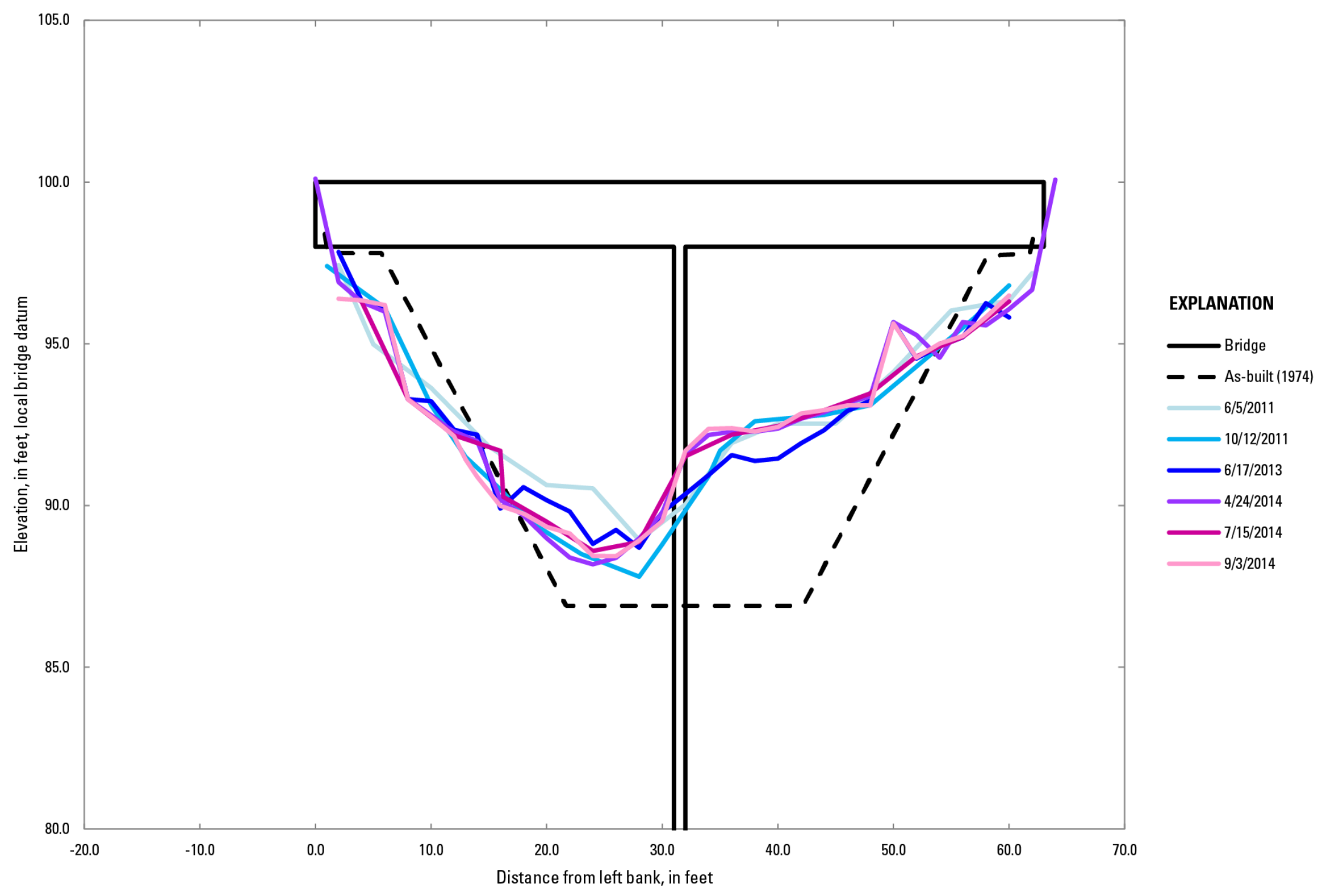

Figure 76. Cross sections showing upstream soundings at bridge 983, Red Cloud River, Alaska, 2011-14. 
BN 983 Red Cloud River, upstream

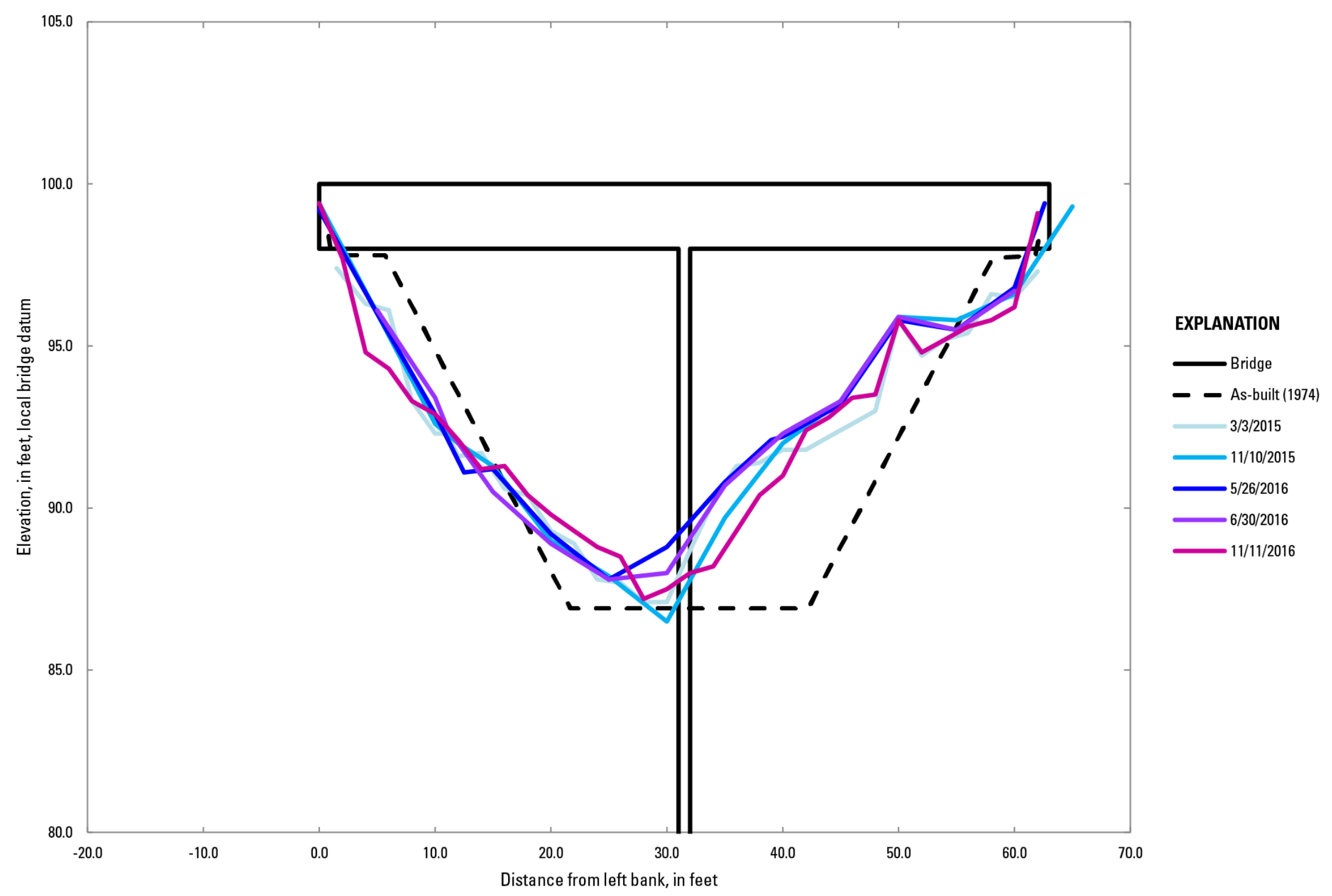

Figure 77. Cross sections showing upstream soundings at bridge 983, Red Cloud River, Alaska, 2015-16. 
BN 983 Red Cloud River, downstream

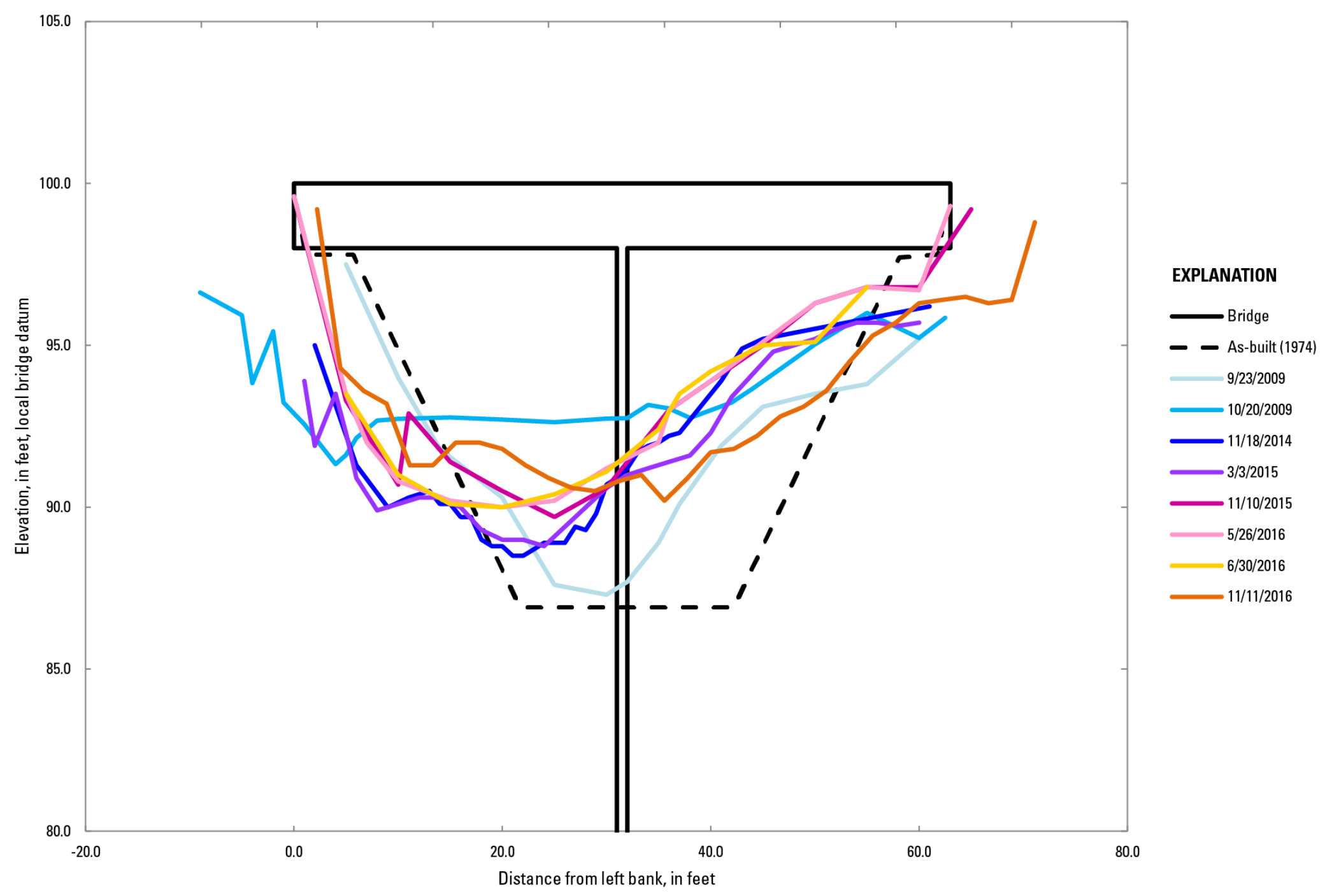

Figure 78. Cross sections showing downstream soundings at bridge 983, Red Cloud River, Alaska, 2009-16. 


\section{Bridge 999, Glacier Creek*}

Glacier Creek is a glacially fed creek originating in the Chugach Range. The channel is braided through the bridge reach at all but the highest flows. The middle piers of the upstream pedestrian bridge and the highway bridge are connected and divide the creek through the bridge. During low-to-moderate flows, the channel is a contained river left of the center pier, where the angle of attack is 25-35 degrees. The channel to the right of the pier has no flow during low flows, but often has ponded water. During moderate-to-high flows, most of the channel is still concentrated on the river left, but the angle of attack on the pier is reduced (0-15 degrees). Cross sections were measured during 1999, 2004, 2005, and 2007-16 (figs. 79 and 80). An overall degradation of $3 \mathrm{ft}$ occurred across the entire channel compared to the as-built cross section, and as much as $6 \mathrm{ft}$ of scour occurred at the right bank pier. 


\section{BN 999 Glacier Creek, upstream}

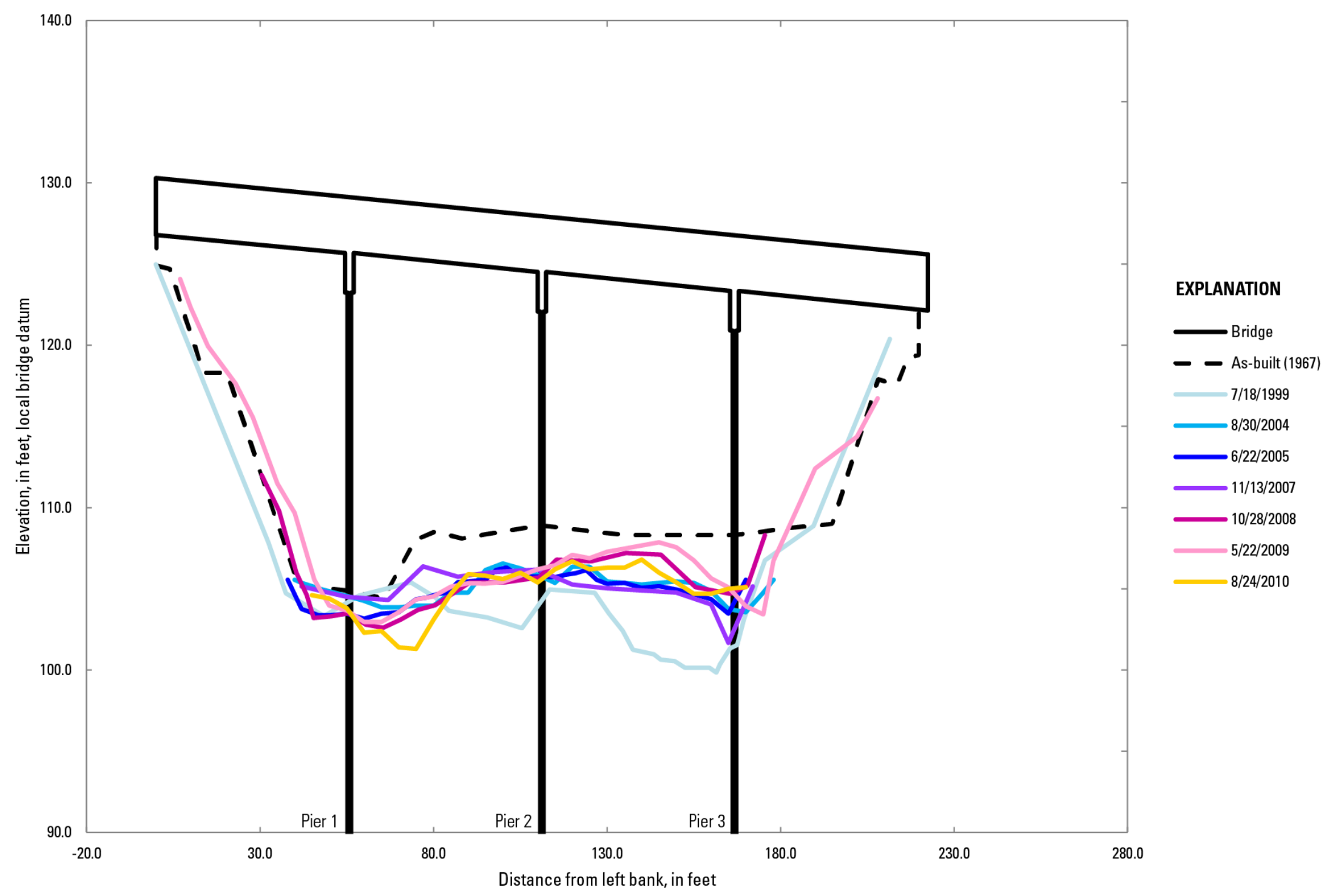

Figure 79. Cross sections showing upstream soundings at bridge 999, Glacier Creek, Alaska, 1999-2010. 
BN 999 Glacier Creek, upstream

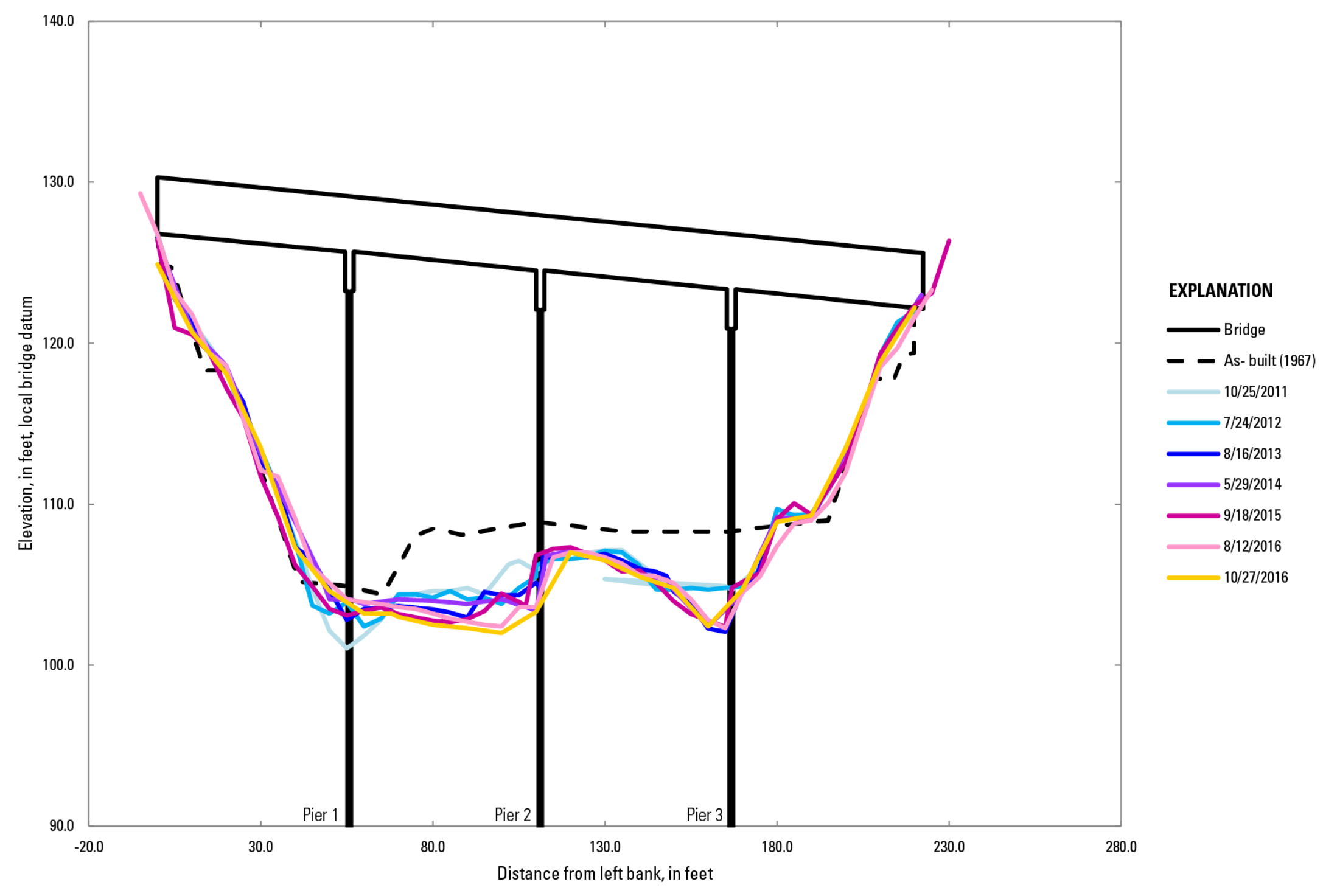

Figure 80. Cross sections showing upstream soundings at bridge 999, Glacier Creek, Alaska, 2011-16. 
Bridge 1243, Nenana River near Windy*

The Nenana River is a meandering single channel that is crossed at a 45 -degree angle by bridge 1243. The bridge is supported by a single pier and the angle of attack on this pier is 10 degrees. The left bank of the channel under the bridge is armored with riprap and the right bank is a steep sandy bank. Cross sections were measured annually from 2007 to 2016 (figs. 81 and 82). The channel has been stable and relatively unchanged during the years in which soundings were measured, with a maximum of $2 \mathrm{ft}$ of localized scour around the pier. 
BN 1243 Nenana River near Windy, upstream

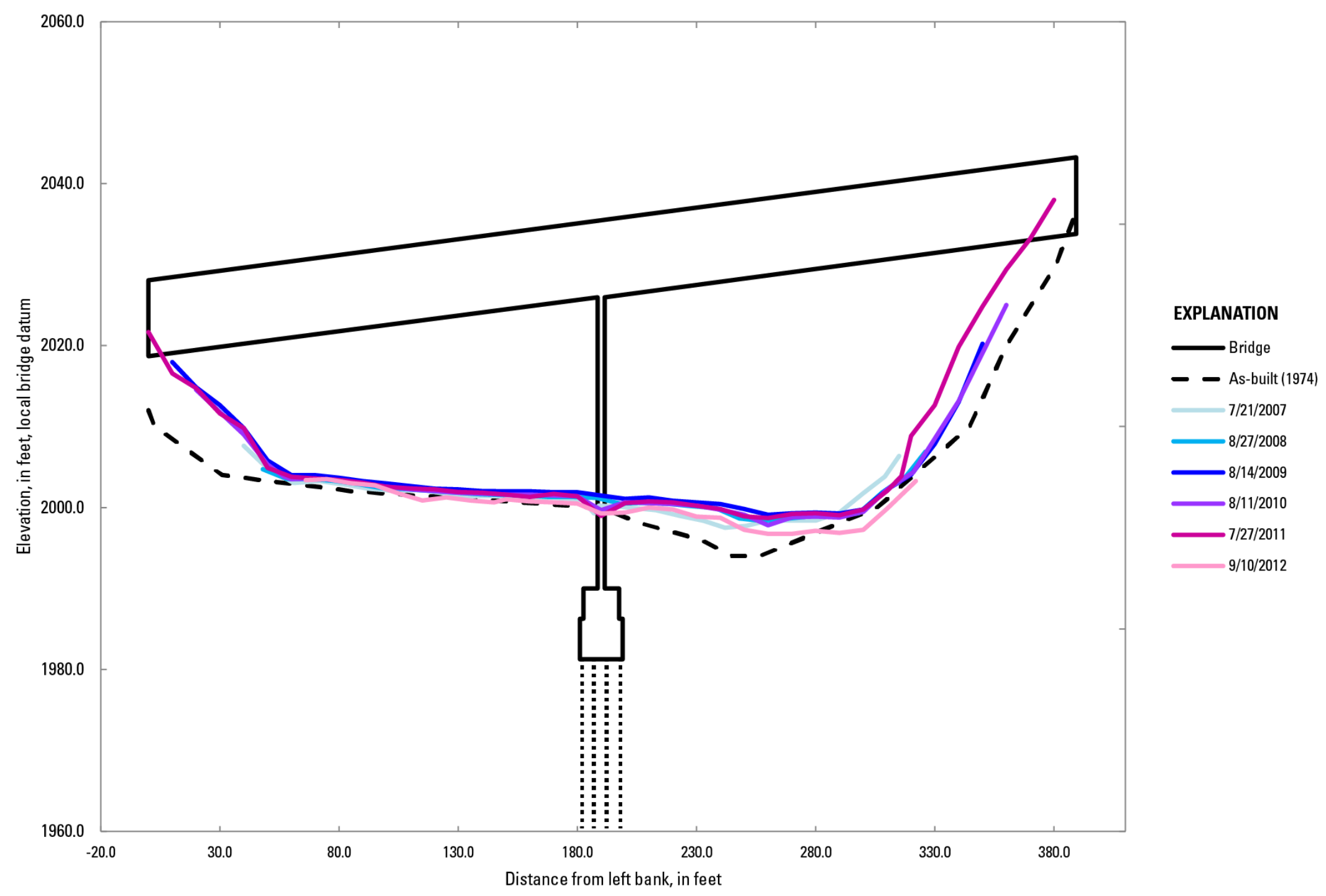

Figure 81. Cross sections showing upstream soundings at bridge 1243, Nenana River near Windy, Alaska, 2007-12. 
BN 1243 Nenana River near Windy, upstream

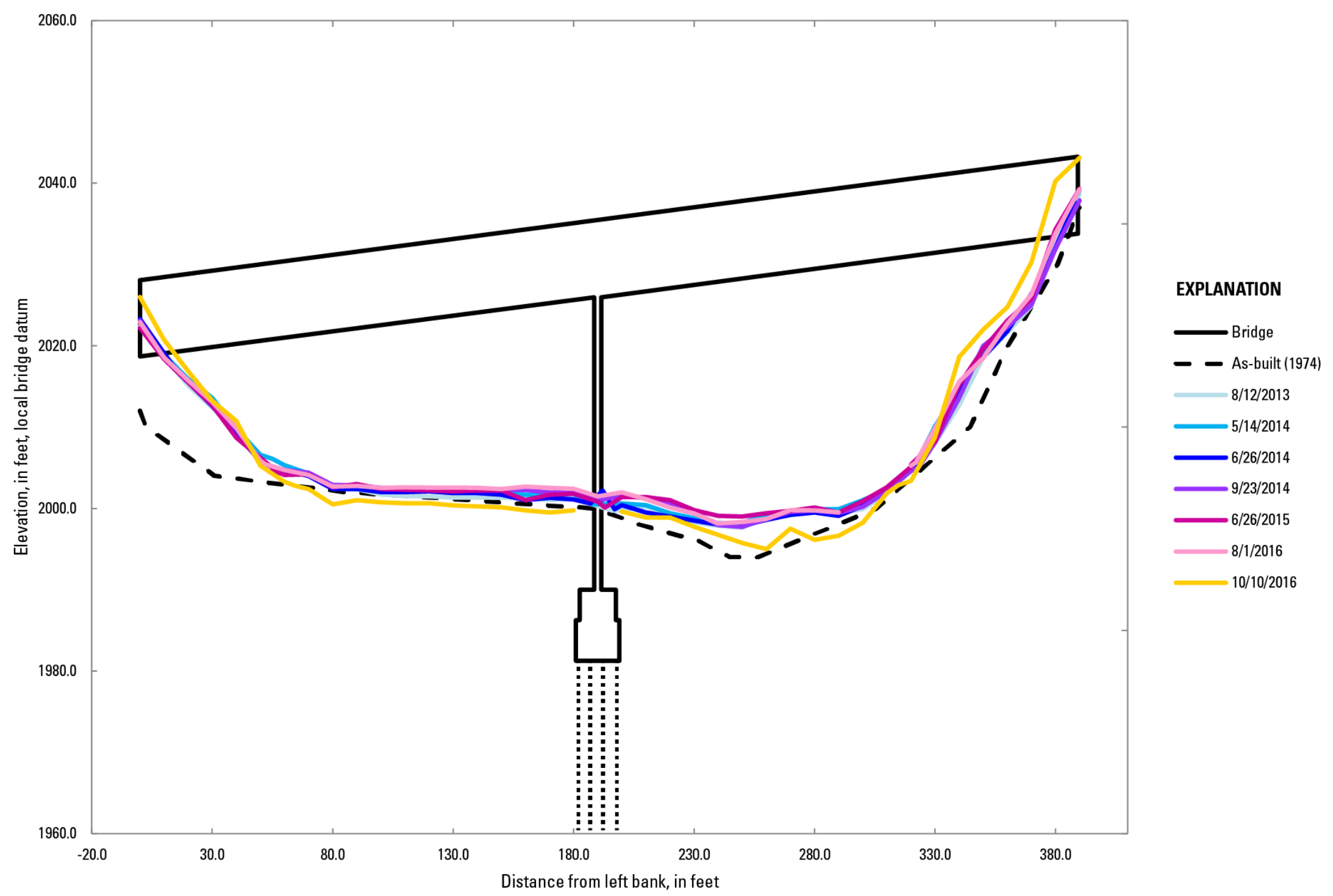

Figure 82. Cross sections showing upstream soundings at bridge 1243, Nenana River near Windy, Alaska, 2013-16. 


\section{Bridge 1383, Lowe River}

The Lowe River is a high-gradient, boulder-strewn channel that originates in the Chugach Mountains from Deserted Glacier before traversing Keystone Canyon where bridge 1383 is located. The right pier typically is out of the water except during floods. The left side of the channel is on bedrock, whereas the road approach is heavily armored with riprap. Flow increases and decreases relatively quickly during heavy rain owing to the steep gradient of the river and its drainage basin. Cross sections were measured during 2005-08 and 2010-16 (figs. 83-85). About $5 \mathrm{ft}$ of aggradation occurred overall across the entire channel compared to the as-built cross section. Between 2005 and 2006, streambed elevation fluctuated $6 \mathrm{ft}$ around the pier, but never decreased below the as-built elevation. Since 2006, the right side of the channel has been relatively stable. 
BN 1383 Lowe River, upstream

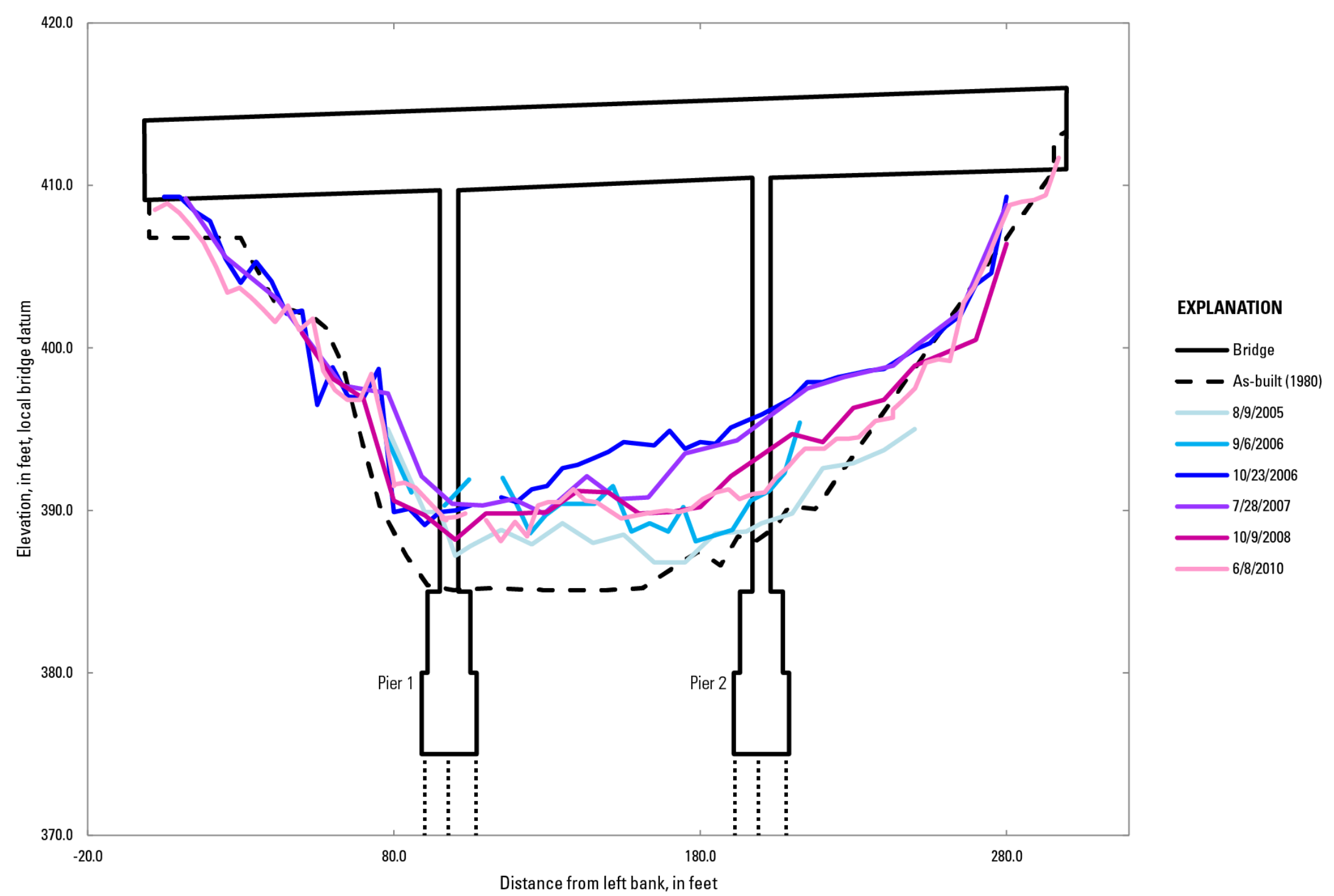

Figure 83. Cross sections showing upstream soundings at bridge 1383, Lowe River, Alaska, 2005-10. 


\section{BN 1383 Lowe River, upstream}

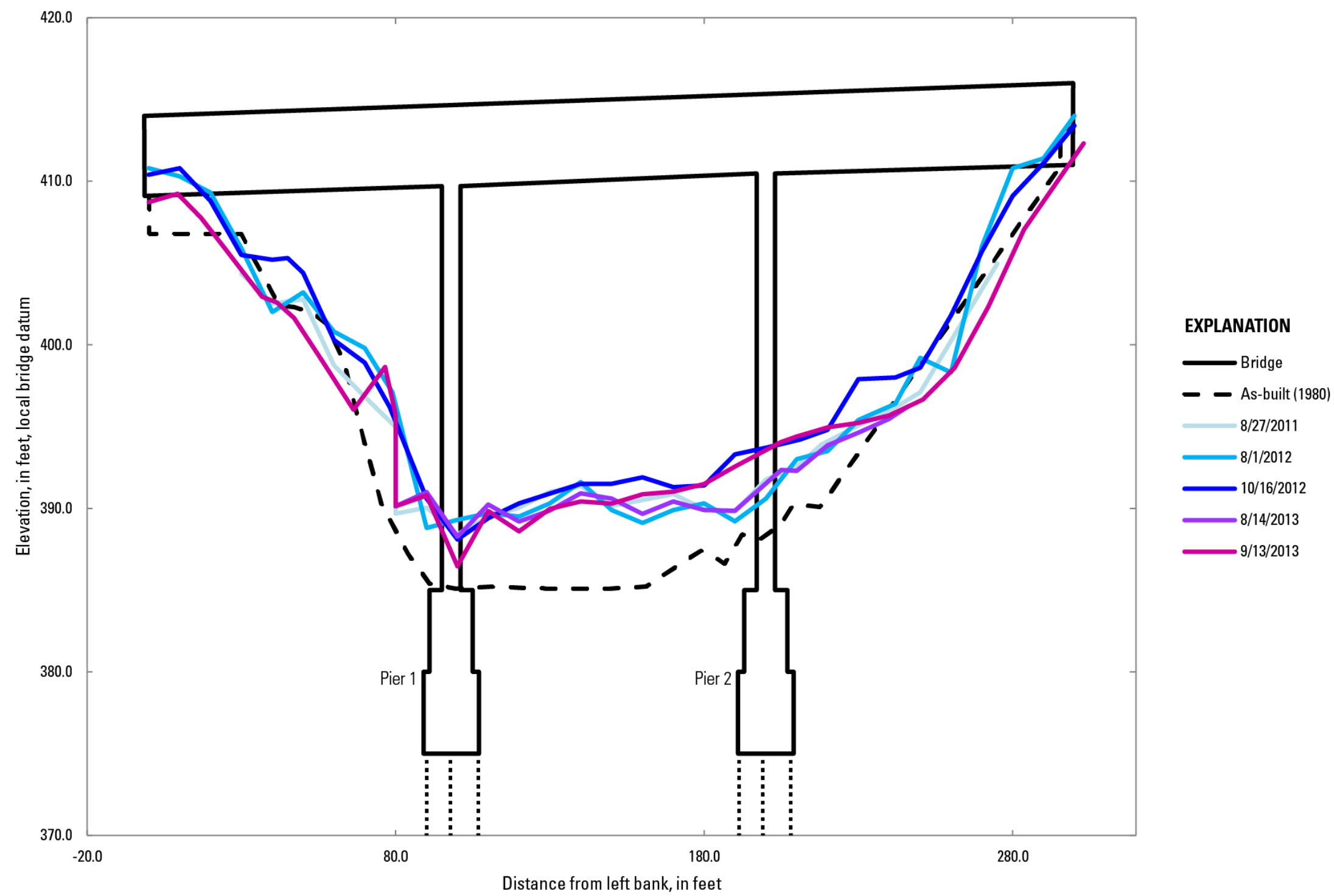

Figure 84. Cross sections showing upstream soundings at bridge 1383, Lowe River, Alaska, 2011-13. 
BN 1383 Lowe River, upstream

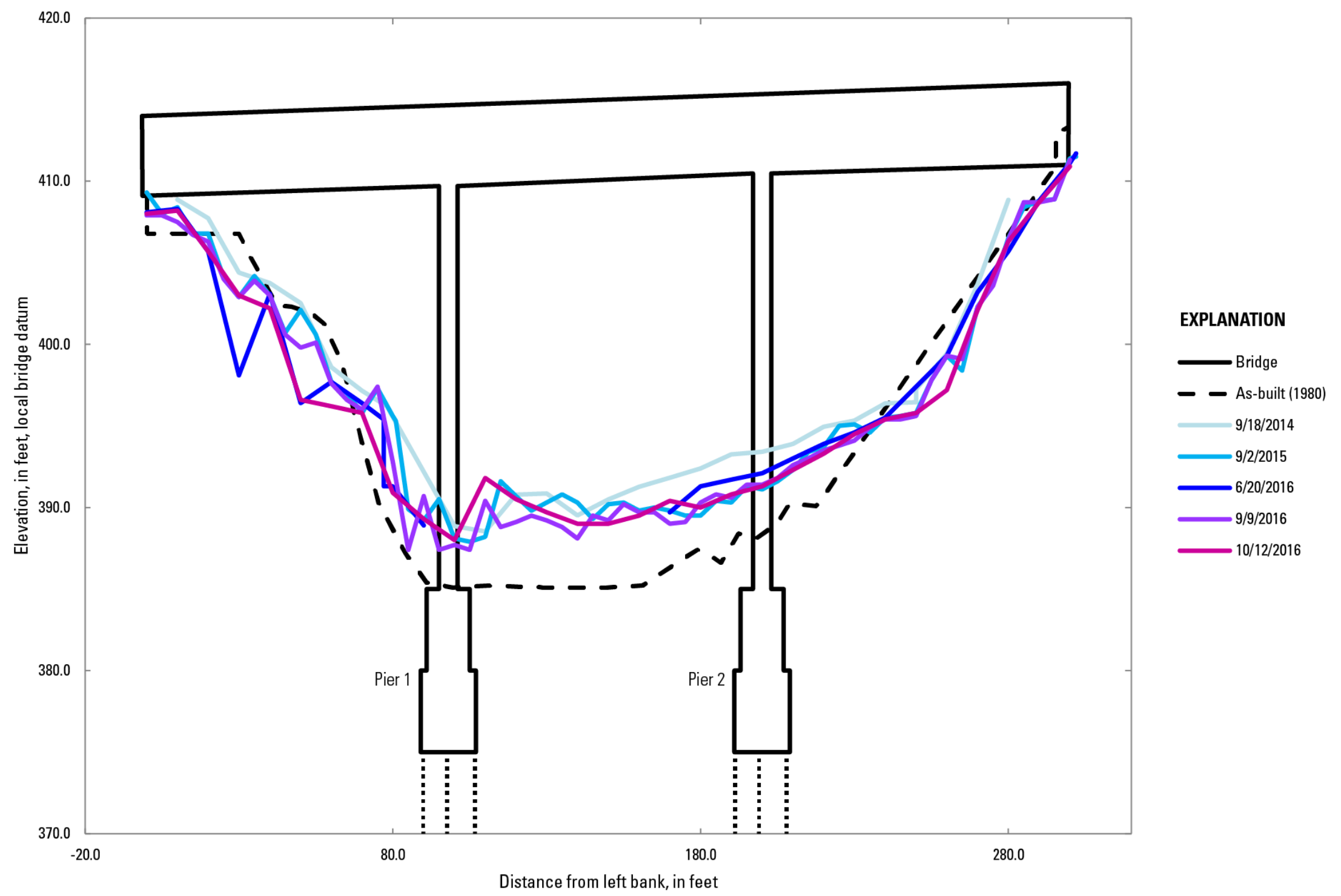

Figure 85. Cross sections showing upstream soundings at bridge 1383, Lowe River, Alaska, 2014-16. 


\section{Summary}

A scour-critical bridge is one with abutment or pier foundations determined to be unstable because of either observed scour or the potential for streambed scour as determined from a scour evaluation. The high gradient, large sediment supply, lack of containment, or relatively frequent overbank floods of rivers and streams in Alaska all contribute to streambed scour at bridges. Changes in streambed elevation over time show that 8 of the 76 monitored rivers are relatively unstable. The data from the continuous soundings can be used to identify long-term aggradation or degradation of the streambed and seasonal changes in streambed elevation, and to fulfill the Federal recommendation for scour monitoring at scour-critical sites.

\section{References Cited}

Arneson, L.A., Zevengergen, L.W., Lagasse, P.F., and Clopper, P.E., 2012, Evaluating scour at bridges (5th ed.): Federal Highway Administration Hydraulic Engineering Circular No. 18, Publication No. FHWA-HIF-12-003, 340 p.

Beebee, R.A., Dworsky, K.L., and Knopp, S.J., 2017, Streambed Scour Evaluations and Conditions at Selected Bridge Sites in Alaska, 2013-15: U.S. Geological Survey Scientific Investigations Report 2017-5149, $67 \mathrm{p}$.

Beebee, R.A., and Schauer, P.V., 2015, Streambed scour evaluations and conditions at selected bridge sites in Alaska, 2012: U.S. Geological Survey Scientific Investigations Report 2015-5154, 45 p.

Brabets, T.P., and Conaway, J.S., 2009, Geomorphology and river dynamics of the lower Copper River, Alaska: U.S. Geological Survey Scientific Investigations Report 2009-5257, 34 p.

Conaway, J.S., 2004, Summary and comparison of multiphase streambed scour analysis at selected bridge sites in Alaska: U.S. Geological Survey Scientific Investigations Report 2004-5066, 34 p.

Conaway, J.S., 2006, Comparison of long-term streambed scour data with modeled values at the Knik River, Alaska, in Proceedings of the Third International Conference on Scour and Erosion, Amsterdam, The Netherlands, November 1-3, 2006: Gouda, The Netherlands, CURNET, p. 146-152.

Conaway, J.S., 2007, Analysis of real-time streambed scour data from bridges in Alaska, in Proceedings of the 2007 World Environmental and Water Resources Congress, Tampa, Florida, May 15-19, 2007: American Society of Civil Engineers, $11 \mathrm{p}$.

Conaway, J.S., 2010, Modeling and monitoring scour during bridge replacement with multi-dimensional modeling and repeated multi-beam surveys at the Tanana River near Tok, Alaska: International Conference on Scour and Erosion, San Francisco, November 7v10, 2010: American Society of Civil Engineers, 3 p.

Conaway, J.S., and Moran, E.H., 2004, Development and calibration of a two-dimensional hydrodynamic model of the Tanana River near Tok, Alaska: U.S. Geological Survey Scientific Investigations Report 2004-1225, 34 p.

Conaway, J.S., and Schauer, P.V., 2012, Evaluation of streambed scour at bridges over tidal waterways in Alaska: U.S. Geological Survey Scientific Investigations Report 2012-5245, 38 p.

Heinrichs, T.A., Kennedy, B.W., Langley, D.E., and Burrows, R.L., 2001, Methodology and estimates of scour at selected bridge sites in Alaska: U.S. Geological Survey Water-Resources Investigations Report 00-4151, 44 p.

Heinrichs, T.A., Langley, D.E., and Burrows, R.L., Conaway, J.S., 2006, Hydraulic survey and scour assessment of bridge 524, Tanana River at Big Delta, Alaska: U.S. Geological Survey WaterResources Investigations Report 2006-5282, 44 p. 
Lagasse, P.F., Zevenbergen, L.W., Spitz, W.J., and Arneson, L.A., 2012, Stream stability at highway structures (4th ed.): Federal Highway Administration Hydraulic Engineering Circular No. 20, Publication No. FHWA-HIF-12-004, 328 p.

Langley, D.E., 2006, Calculation of scour depth at the Parks highway bridge on the Tanana River at Nenana, Alaska, using one- and two-dimensional hydraulic models: U.S. Geological Survey Scientific Investigations Report 2006-5023, 34 p.

Norman, V.W., 1975, Scour at selected bridge sites in Alaska: U.S. Geological Survey Water-Resources Investigations Report 32-75, 160 p. 


\section{Appendix 1. Tabular Data for All Cross Sections Collected for this Study}

Exact stations of cross sections along the bridge (in feet) starting from the left side of the bridge and the streambed elevations (in feet) at those stations are shown in appendix 1. For each cross section, a date and a location indicate whether the measurements were taken either upstream (US) or downstream (DS) of the bridge. If US or DS is not applicable, then the column states Na. The elevations are relative to the bridge deck centerline elevation, except for bridges that are missing as-built plans. In those cases, the reference of streambed measurements is a bridge deck centerline elevation of $100 \mathrm{ft}$. Cross sections that are missing data are stations where either the velocities were too high to obtain a measurement or debris made it too difficult to get an accurate elevation measurement.

Appendix 1 is available for download in Microsoft ${ }^{\circledR}$ Excel format at https://doi.org/10.3133/ofr20191028. 


\section{Appendix 2. Cross Sections of Bed Elevation and Bridge Geometry for Bridges Not Discussed in this Report}

The remainder of the repeated cross sections that are not discussed in this report are shown in appendix 2, which is available for download in Adobe Acrobat ${ }^{\circledR}$ format at https://doi.org/10.3133/ofr20191028. 
Publishing support provided by the U.S. Geological Survey Science Publishing Network, Tacoma Publishing Service Center

For more information concerning the research in this report, contact the Director, Alaska Science Center

U.S. Geological Survey

4210 University Drive

Anchorage, Alaska 99508

https://www.usgs.gov/centers/asc/ 
몸.

흠

范. 